\title{
An Integral Representation of Singular Solutions and Removable Singularities of Solutions to Linear Partial Differential Equations
}

\author{
By \\ Sunao ŌUCH*
}

\section{§ 0. Introduction}

Let $L\left(z, \partial_{z}\right)$ be a linear partial differential operator with the order $m \geqq 1$, whose coefficients are holomorphic in $\Omega=\left\{z \in C^{n+1} ;|z| \leqq R\right\}$, and $K$ be a connected nonsingular complex hypersurface in $\Omega$ through the origin $z=0$. In the present paper we treat the equation

$$
L\left(z, \partial_{z}\right) u(z)=f(z),
$$

where $u(z)$ may be singular on $K$, and $f(z)$ is holomorphic in $\Omega$.

There are two main purposes in this paper. The one is to give an integral representation of solutions to (0.1) singular on $K$ (Theorem 2.5). The other is to show that if $u(z)$ has some growth property near $K$ under some conditions on $L\left(z, \partial_{z}\right)$, then $u(z)$ is holomorphic at $K$ (Theorem 1.3), that is, the singularity on $K$ is removable. The conditions on $L\left(z, \partial_{z}\right)$ are given by means of the characteristic indices of $K$ and the localization on $K$ defined in [9] and [10]. The author does not know such a theorem about removable singularities of solutions to linear partial differential equations as that in this paper. In order to show Theorem 1.3 we need the detailed analysis of the obtained integral representation and use theorems about the Laplace transform of functions with asymptotic expansions with bounds (Theorems 1.7 and 1.9), which are also the results of this paper.

We make reference to singular solutions to (0.1) in short. As for existence,

Communicated by M. Kashiwara, October 1, 1987.

* Department of Mathematics, Sophia University, Tokyo 102, Japan. 
it was studied in [1], [2], [3], [13], [16] and others, where they constructed singular solutions to noncharacteristic Cauchy problem with singular initial data. The existence of solutions singular on $K$ was also considered in [4], [11] and [14] apart from singular Cauchy problems. The condition in [11], given by means of the principal localization, is less restrictive than those in others.

The integral representation was obtained for operators with decomposable principal symbol in [6] and [7]. In the present paper we give it for a wider class of operators and its form is slightly different from that given in [6] and [7].

In $\S 1$ we give notations and definitions and state the theorem concerning removable singularities. We also give results about the relations between the Laplace transform and functions with asymptotic expansion with bounds. We don't give here the integral representation. Because it requires further preliminaries. In $\S 2$ we give them and the integral representation. Roughly speaking its kernel function takes the form

$$
K\left(z, \lambda, t^{\prime \prime}\right)=\int \exp \left(-\lambda^{\alpha} \zeta\right) w\left(z, t^{\prime \prime}, \lambda, \zeta\right) d \zeta .
$$

So construction of representation is that of $w\left(z, t^{\prime \prime}, \lambda, \zeta\right)$, which we call also the kernel function. In $\S 3-\S 5$ we construct $w\left(z, t^{\prime \prime}, \lambda, \zeta\right)$ and get the integral representation of solutions singular on $K$. In $\S 6$ we show lemmas and propositions used in the previous sections or required in the following sections. In $\S 7$ we investigate the kernel function $w\left(z, t^{\prime \prime}, \lambda, \zeta\right)$. We try to analyze its singularities with respect to $\zeta$. The estimates of functions appearing in $\S 3-\S 7$ are given in $\$ 9$. In $\S 8$ we complete the proof of the theorem of removable singularities (Theorem 1.3), combining the results obtained in the preceding sections with Theorems 1.7 and 1.9. In $§ 9$ we show what are left unproved, in which the estimates and some lemmas of holomorphic functions needed in construction of the integral representation are contained. In $\S 10$ we discuss about functions with asymptotic expansions and give the proofs of Theorems 1.7 and 1.9 .

In this paper many constants will appear. So for simplicity we denote various constants by the same $A, B, C$, etc.. There will be no confusions.

\section{$\S 1 . \quad$ Notations and Definitions}

First we give notations. $z=\left(z_{0}, z_{1}, \cdots, z_{n}\right)=\left(z_{0}, z^{\prime}\right)=\left(z_{0}, z_{1}, z^{\prime \prime}\right)$ is the coordinate of the $(n+1)$-dimensional complex space $\mathbb{C}^{n+1}$ with the norm $|z|=\max$ $\left\{\left|z_{i}\right| ; 0 \leqq i \leqq n\right\}$, while $\xi=\left(\xi_{0}, \xi_{1}, \cdots, \xi_{n}\right)=\left(\xi_{0}, \xi_{1}, \xi^{\prime \prime}\right)=\left(\xi_{0}, \xi^{\prime}\right)$ is the dual 
variable. $\partial_{z}=\left(\partial_{0}, \partial_{1}, \cdots, \partial_{n}\right)=\left(\partial_{0}, \partial_{1}, \partial^{\prime \prime}\right)=\left(\partial_{0}, \partial^{\prime}\right), \partial_{i}=\partial / \partial z_{i}$ is the differentiation. $\boldsymbol{N}$ is the set of natural numbers and $\boldsymbol{Q}$ is the set of rational numbers. Now let $K$ be a nonsingular complex hypersurface through the origin $z=0$. We may choose the coordinate so that $K=\left\{z_{0}=0\right\}$. Then we can write $L\left(z, \partial_{z}\right)$ in $(0.1)$ in the form

$$
\left\{\begin{array}{l}
L\left(z, \partial_{z}\right)=\sum_{k=0}^{m} L_{k}\left(z, \partial_{z}\right), \\
L_{k}\left(z, \partial_{z}\right)=\sum_{i=s_{k}}^{k} A_{k, i}\left(z, \partial^{\prime}\right)\left(\partial_{0}\right)^{k-i}
\end{array}\right.
$$

Here $L_{k}\left(z, \partial_{z}\right)$ is the homogeneous part of degree $k$. The integers $s_{k}(0 \leqq k \leqq m)$ are chosen so that $A_{k, s_{k}}\left(z, \xi^{\prime}\right) \neq 0$ if $L_{k}(z, \xi) \neq 0$, and we put $s_{k}=+\infty$ if $L_{k}(z, \xi)$ $\equiv 0$.

Now let us give several definitions and notions derived from $L\left(z, \partial_{z}\right)$. Put $A=\left\{\left(k, s_{k}\right) \in \boldsymbol{R}^{2} ; 0 \leqq k \leqq m, s_{k} \neq+\infty\right\}$. We denote the convex hull of $A$ by $\hat{A}$. Let $\Sigma$ be the lower convex part of the boundary of $\hat{A}$, and $\Delta$ be the vertices of $\Sigma$. We set $\Delta=\left\{\left(k_{i}, s_{k_{i}}\right) ; 0 \leqq i \leqq l\right\}, m=k_{0}>k_{1}>k_{2}>\cdots>k_{l} \geqq 0$. If $l=0, \Sigma=$ $\Delta=\left\{\left(m, s_{m}\right)\right\}$. Assume $l \geqq 1$. Then $\Sigma$ consists of segments $\Sigma(\mathrm{i})(1 \leqq i \leqq l)$. The end points of $\Sigma(\mathrm{i})$ are $\left(k_{i-1}, s_{k_{i-1}}\right)$ and $\left(k_{i}, s_{k_{i}}\right)$ (see Fig. 1.1).

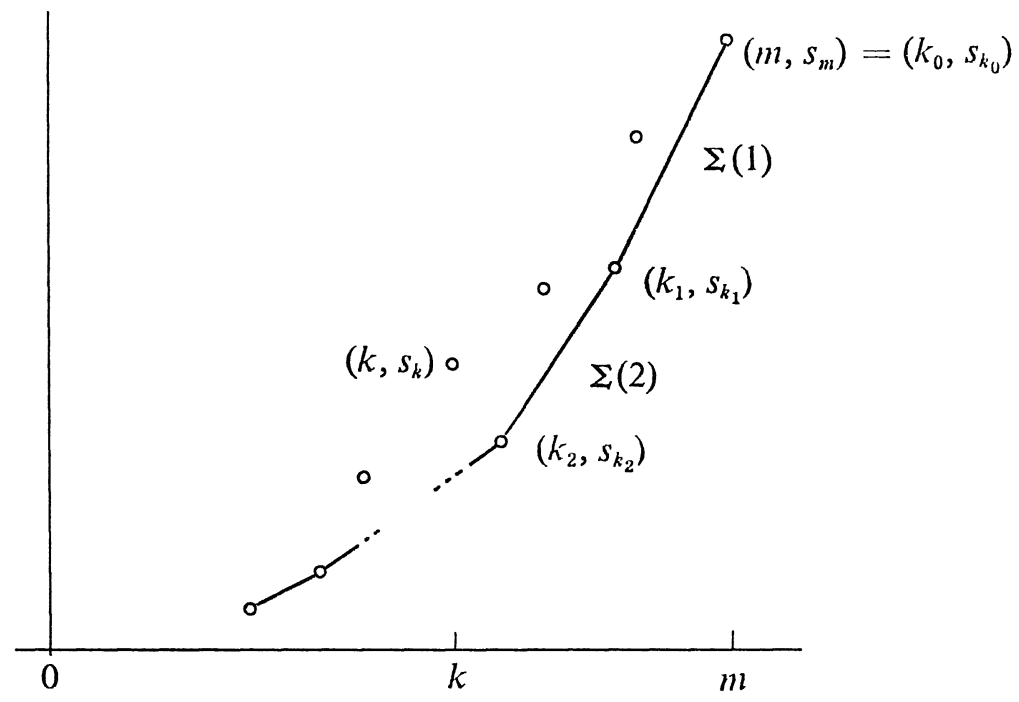

Fig. 1.1.

Set

$$
\left\{\begin{array}{l}
\sigma_{0}=+\infty, \\
\sigma_{i}=\max \left\{1,\left(s_{k_{i-1}}-s_{k_{i}}\right) /\left(k_{i-1}-k_{i}\right)\right\} \quad(1 \leqq i \leqq l)
\end{array}\right.
$$

Then there is a $p \in N$ such that 


$$
+\infty=\sigma_{0}>\sigma_{1}>\sigma_{2}>\cdots>\sigma_{p-1}>\sigma_{p}=1 .
$$

Define for $0 \leqq i \leqq p$

$$
\left\{\begin{array}{l}
\alpha_{i}=\left(\sigma_{i}-1\right) / \sigma_{i}, \\
\gamma_{i}=\sigma_{i}-1 .
\end{array}\right.
$$

Here we mean $\alpha_{0}=1$ and $\gamma_{0}=+\infty$. From the definition of $\sigma_{i}$, we have

Lemma 1.1. Suppose $s_{k} \neq+\infty$. Then there are nonnegative $\beta_{k}^{i} \in \mathbb{Q}(1 \leqq$ $i \leqq p$ ) such that

$$
\left(s_{k_{i-1}}-s_{k}\right)\left(1-\alpha_{i}\right)+\beta_{k}^{i}=k_{i-1}-k,
$$

and $\left(k, s_{k}\right) \in \Sigma(i)$ if and only if $\beta_{k}^{i}=0$.

The proof of Lemma 1.1 will be given in $\$ 6$ and we set in the sequel $\beta_{k}=\beta_{k}^{1}$ and $\alpha=\alpha_{1}$ for simplicity.

Remark 1.2. In [9] and [10] (see also [12]) characteristic indices were defined and denoted also by $\left\{\sigma_{i}\right\}$. In general they are different from those defined by (1.2). But if we assume some conditions on $L\left(z, \partial_{z}\right)$, they are coincident with each other (see Remark 1.4).

For an open set $W$ in $\boldsymbol{C}^{N}, \tilde{W}$ means the universal covering space of $W$. We denote by $\mathcal{O}(W)$ the set of all holomorphic functions on $W$ and by $\mathcal{O}(\tilde{W})$ the set of all holomorphic functions on $\tilde{W}$. Let $U$ be a polydisk in $\mathbb{C}^{n+1}$ with center $z=0$. Then we set $\widetilde{U}(a, b)=\left\{z \in\left(\widetilde{U-\left\{z_{0}=0\right\}}\right) ; a<\arg z_{0}<b\right\}$ and $\tilde{U}(a)=\tilde{U}(-a, a)(a>0)$. Hence $\mathcal{O}(U)$ is the set of all holomorphic functions in $U$ and $\mathcal{O}(\widetilde{U}(a, b))$ is the set of all holomorphic functions on the sector $\tilde{U}(a, b)$. Obviously if $b-a>2 \pi$, then $\mathcal{O}(\widetilde{U}(a, b))$ contains multi-valued functions.

Now, by using these definitions, we can give a theorem about the removablity of singularity $K$, which is one of the main results in this paper.

Theorem 1.3. Assume

$$
\left\{\begin{array}{l}
\text { (a) } \sigma_{1}>1, \\
\text { (b) } s_{k_{p-1}}=0, \\
\text { (c) } \prod_{i=0}^{p-1} A_{k_{i}, s_{k_{i}}}\left(0, z^{\prime}, \xi^{\prime}\right) \neq 0 .
\end{array}\right.
$$

Let $u(z) \in \mathcal{O}\left(\tilde{\Omega}\left(\theta_{0}\right)\right)\left(\theta_{0}>\pi\left(1 / 2 \gamma_{p-1}+1\right)\right)$ be a solution to

$$
L\left(z, \partial_{z}\right) u(z)=f(z) \in \mathcal{O}(\Omega) .
$$

Suppose that for any $\varepsilon>0$, there is a $C_{\varepsilon}>0$ such that 


$$
|u(z)| \leqq C_{\varepsilon} \exp \left(\varepsilon\left|z_{0}\right|^{\left.-\gamma_{p-1}\right)} \quad \text { for } \quad z \in \widetilde{\Omega}\left(\theta_{0}\right) .\right.
$$

Then $u(z) \in \mathcal{O}(\Omega)$.

The proof of Theorem 1.3 is long and completed in $\S 8$. As we said in $\S 0$, Theorem 1.3 follows from the integral representation in $\$ 2$.

Remark 1.4. Let $L\left(z, \partial_{z}\right)$ be an operator satisfying (1.6)-(c). Then $\left\{\sigma_{i}\right\}$ $(1 \leqq i \leqq p)$ are coincident with the characteristic indices in [9] and [10], and $A_{k_{i}}$ $\left(0, z^{\prime}, \xi^{\prime}\right)(0 \leqq i \leqq p-1)$ are the localizations defined there. So we can state (1.6) (a)-(c) in other conditions which are invariant under the coordinates transformations.

We give simple examples.

Corollary 1.5. Let

$$
L\left(z, \partial_{z}\right)=\left(\partial_{0}\right)^{k}+A\left(z, \partial^{\prime}\right),
$$

where $A\left(z, \partial^{\prime}\right)$ is an operator with ord. $A\left(z, \partial^{\prime}\right)=m>k$ and the principal symbol $A_{m}\left(z, \xi^{\prime}\right)$. Assume $A_{m}\left(0, z^{\prime}, \xi^{\prime}\right) \neq 0$. Then if a solution $u(z) \in \mathcal{O}\left(\tilde{\Omega}\left(\theta_{0}\right)\right)$ to (1.7), $\left(\theta_{0}>(\pi / 2)(m / k+1)\right)$, satisfies for any $\varepsilon>0$

$$
|u(z)| \leqq C_{\varepsilon} \exp \left(\varepsilon\left|z_{0}\right|^{-k /(m-k)}\right) .
$$

Then $u(z) \in \mathcal{O}(\Omega)$.

We have $\sigma_{1}=m /(m-k)$ and $\sigma_{2}=1$. Hence $r_{1}=\sigma_{1}-1=k /(m-k)$ for $L\left(z, \partial_{z}\right)$ in (1.9). More concretely let $L\left(z, \partial_{z}\right)=\left(\partial_{0}\right)^{k}-(-1)^{m+k}\left(\partial_{1}\right)^{m}$. Set

$$
u_{1}(z)=\int_{0}^{+\infty} \exp \left(-\lambda z_{0}-\lambda^{k / m}\left(z_{1}+d\right)\right) d \lambda,(d>0) .
$$

$u_{1}(z)$ satisfies $L\left(z, \partial_{z}\right) u_{1}(z)=0$. It holds for $u_{1}(z)$ that for any $\varepsilon>0$ if $z \in\{z ; \mid \arg$ $\left.z_{0}\left|<\frac{\pi}{2}\left(\frac{m}{k}+1\right)-\varepsilon,\right| z_{1} \mid<r_{\varepsilon}\right\},|u(z)| \leqq C_{\varepsilon}$. So the condition $\theta_{0}>\frac{\pi}{2}\left(\frac{m}{k}+1\right)$ is essential. $u_{1}(z)$ has the bound $\left|u_{1}(z)\right| \leqq A \exp \left(B\left|z_{0}\right|^{-k /(m-k)}\right)$ on $\{z ;|z|<r$, $\left.-\infty<\arg z_{0}<+\infty\right\}$. The condition (1.10) is also essential.

Now let us proceed to give the theorems about functions with asymptotic expansions. As we said in $\S 0$, they will be used to show Theorem 1.3. The proofs are in $\S 10$. Let $u(t)$ be a continuous function on $[A,+\infty)(A>0)$ such that $|u(t)| \leqq C \exp \left(B|t|^{\gamma}\right)(\gamma>0)$. We define the $r$-Laplace transform $\hat{u}(\xi)$ of $u(t)$ by

$$
\hat{u}(\xi)=\int_{a}^{+\infty} \exp (\xi t) u\left(t^{1 / \gamma}\right) t^{-1} d t \quad\left(a>A^{\gamma}\right)
$$


which is holomorphic in $\{\xi ; \operatorname{Re} \xi<-B\}$. The inversion formula is given by

$$
u(t)=\frac{t^{\gamma}}{2 \pi i} \int_{d-i \infty}^{d+i \infty} \exp \left(-\xi t^{\gamma}\right) \hat{u}(\xi) d \xi \quad(d<-B)
$$

Definition 1.6. We say that $u(t)$ has the $r$-asymptotic expansion on $[A,+\infty)$, if for any $N \geqq 1$

$$
\left|u(t)-\sum_{k=0}^{N-1} c_{k} t^{-k}\right| \leqq A_{1} R^{-N} \Gamma(N / \gamma+1)|t|^{-N}
$$

holds on $[A,+\infty)$.

From the definition $\left|c_{N}\right| \leqq A_{1} R^{-N} \Gamma(N / \gamma+1)$, that is, the coefficients of the asymptotic expansion have the estimates of Gevrey type. Suppose that $u(t)$ has the asymptotic expansion (1.14). Then, by using the sequence $\left\{c_{k}\right\} \quad(k=0$, $1, \cdots)$, define

$$
g(z)=\sum_{k=0}^{+\infty} \frac{c_{k} z^{k}}{\Gamma(k / \gamma+1)},
$$

which is holomorphic in $\left\{z \in C^{1} ;|z|<R\right\}$. We have for $\hat{U}(\xi)$

Theorem 1.7. Assume $u(t)$ has the r-asymptotic expansion (1.14) on $[A,+\infty)$. Then the $r$-Laplace transform $\hat{u}(\xi)$ is holomorphic in $\left(\left\{\xi ; \operatorname{Re} \xi<R^{\gamma}\right.\right.$, $\left.\xi \notin\left[0, R^{\gamma}\right)\right\}$ and it can be holomorphically extended into $\left\{\xi ; 0<|\xi|<R^{\gamma}\right\}$ such that $\hat{u}(\xi) \in \mathcal{O}\left(\left\{\overline{\left\{\xi 0<|\xi|<R^{\gamma}\right\}}\right)\right.$, for any $\Theta>0$ and $0<c<R^{\gamma}$

$$
|\hat{u}(\xi)| \leqq M_{c, \Theta}|\log \xi| \text { in }\{\xi ;|\arg \xi|<\Theta, 0<|\xi|<c\} \text {, }
$$

and $\left\{\hat{u}(\xi)-\hat{u}\left(\xi e^{2 \pi i}\right)\right\} / 2 \pi i=g\left(\xi^{1 / \gamma}\right)$, where $\xi^{1 / \gamma}=|\xi|^{1 / \gamma} e^{i(\arg \xi) / \gamma}$.

Now let us consider functions on a sector with asymptotic expansions with bounds. We set $\tilde{S}(a, b)=\left\{t \in\left(\widetilde{C^{1}-\{0\}} ;|t| \geqq A, a<\arg t<b\right\} \quad(A>0)\right.$ and $\tilde{S}(a)=\tilde{S}(-a, a)(a>0)$.

Definition 1.8. We say that $u(t) \in \mathcal{O}(\tilde{S}(a, b))$ has the r-asymptotic expansion

$$
u(t) \sim \sum_{k=0}^{+\infty} c_{k} t^{-k} \text { at } t=\infty \text { in } \widetilde{S}(a, b),
$$

if for any $N>0$

$$
\left|u(t)-\sum_{k=0}^{N-1} c_{k} t^{-k}\right| \leqq A_{1} B_{1}^{N} \Gamma(N / \gamma+1)|t|^{-N}
$$

holds on any closed subsectors $S_{1}$ in $\tilde{S}(a, b)$.

If $u(t) \in \mathcal{O}\left(\tilde{S}\left(\theta_{0}\right)\right)$ satisfies, for any $\varepsilon>0$ 


$$
|u(t)| \leqq C_{\varepsilon} \exp \left(\varepsilon|t|^{\gamma}\right)(\gamma>0) \text { in } \widetilde{S}\left(\theta_{0}\right),
$$

then $\hat{u}(\xi) \in \mathcal{O}\left(\left\{\xi ;|\arg \xi-\pi|<r \theta_{0}+\pi / 2\right\}\right)$. We have

Theorem 1.9. Assume $u(t) \in \mathcal{O}\left(\tilde{S}\left(\theta_{0}\right)\right)$ satisfies (1.19). Then $u(t)$ has the $\gamma$-asymptotic expansion

$$
u(t) \sim \sum_{k=0}^{+\infty} c_{k} t^{-k} \quad \text { at } t=\infty \text { in } \tilde{S}\left(\theta_{0}\right),
$$

if and only if the $r$-Laplace transform $\hat{u}(\xi) \in \mathcal{O}\left(\left\{\xi ;|\arg \xi-\pi|<r \theta_{0}+\pi / 2\right\}\right)$ satisfies the following conditions:

$\hat{u}(\xi)$ is holomorphically extensible into $\{\xi ; 0<|\xi|<c\}$ for some $c>0$ so that $\hat{u}(\xi) \in \mathcal{O}(\widehat{\{\xi ; 0<|\xi|<c\}})$, for any $\Theta>0$

$$
|\hat{u}(\xi)| \leqq M_{\Theta}|\log \xi| \quad \text { in }\{\xi ;|\arg \xi|<\Theta, 0<|\xi|<c\},
$$

and $F(\xi)=\left\{\hat{u}(\xi)-\hat{u}\left(\xi e^{2 \pi i}\right)\right\} / 2 \pi$ i has the convergent power series of $\xi^{1 / \gamma}$ at $\xi=0$,

$$
F(\xi)=\sum_{k=0}^{+\infty} c_{k} \xi^{k / \gamma} / \Gamma(k / \gamma+1)\left(|\xi|^{1 / \gamma}<R\right) \quad\left(c \leqq R^{\gamma}\right) .
$$

Moreover if $\hat{u}(\xi) \in \mathcal{O}\left(\left\{\xi ;|\arg \xi-\pi|<\gamma \theta_{0}+\pi / 2\right\}\right)$ satisfies all above conditions and $\theta_{0}>\pi / 2 \gamma+\pi$, then $u(t)$ is holomorphic at $t=\infty$.

For functions with asymptotic expansions with Gevrey type we refer to [15], where ordinary differential equations were treated, and the papers in its references.

\section{§ 2. Integral Representation}

In $\S 2$ we show an integral representation of a singular solution $u(z)$ satisfying (0.1). From now on, we always assume $u(z) \in \mathcal{O}\left(\tilde{\Omega}\left(\theta_{0}\right)\right)\left(\theta_{0}>\pi\right)$, $s_{m} \geqq 1$ and

$$
A_{m, s_{m}}\left(0, \xi^{\prime}\right) \neq 0
$$

So $K$ is characteristic. We may assume that for $\xi^{\prime}=\hat{\xi}^{\prime}=(1,0, \cdots, 0)$

$$
A_{m, s_{m}}\left(0, \hat{\xi^{\prime}}\right) \neq 0 \quad\left(s_{m} \geqq 1\right) .
$$

We try to obtain an integral representation of $u(z)$ as the sum of functions of the form

$$
\left\{\begin{array}{l}
\frac{1}{2 \pi i} \int_{\Lambda(\psi)} \exp \left(\lambda z_{0}\right)(\log \lambda) d \lambda \int_{T^{\prime \prime}} K_{\theta}^{h}\left(z, \lambda, t^{\prime \prime}\right) \hat{u}_{\theta}^{h}\left(\lambda, t^{\prime \prime}\right) d t^{\prime \prime} \\
K_{\theta}^{h}\left(z, \lambda, t^{\prime \prime}\right)=\int_{C(\theta)} \exp \left(-\lambda^{\alpha} \zeta\right) w^{h}\left(z, t^{\prime \prime}, \lambda, \zeta\right) d \zeta,\left(0 \leqq h \leqq s_{m}-1\right) .
\end{array}\right.
$$


The path $\Lambda(\psi), T^{\prime \prime}$ and $C(\theta)$, and the functions $\hat{u}_{\theta}^{h}\left(\lambda, t^{\prime \prime}\right)$ and $w^{h}\left(z, t^{\prime \prime}, \lambda, \zeta\right)$ are determined in the following. In order to do so we need some preliminaries. All the proofs are given in the later sections.

Now let us explain the functions in (2.2) and the paths of integration. The explanations are divided into 3 parts.

(I) The definitions of $\hat{u}_{\theta}^{h}\left(\lambda, t^{\prime \prime}\right)$ and the path $\Lambda(\psi)$. Consider the traces of $u(z) \in \mathcal{O}\left(\widetilde{\Omega}\left(\theta_{0}\right)\right), \Omega=\left\{z \in \mathbb{C}^{n+1} ;|z| \leqq R\right\}$, to $z_{1}=0$,

$$
u^{h}\left(z_{0}, z^{\prime \prime}\right)=\left(\partial / \partial z_{1}\right)^{h} u\left(z_{0}, 0, z^{\prime \prime}\right), \quad\left(0 \leqq h \leqq s_{m}-1\right),
$$

and define

$$
\hat{u}_{\theta}^{h}\left(\lambda, t^{\prime \prime}\right)=\frac{1}{2 \pi i} \int_{T(\theta)} \exp \left(-\lambda t_{0}\right) u^{h}\left(t_{0}, t^{\prime \prime}\right) d t_{0} .
$$

$T(\theta)\left(-\theta_{0}<\theta<\theta_{0}-2 \pi\right)$ is a path starting at $R \mathrm{e}^{i(\theta+2 \pi)}$, going to $\varepsilon e^{i(\theta+2 \pi)}(0<\varepsilon<R)$, rounding the origin once on $\left|t_{0}\right|=\varepsilon$ and ending at $R e^{i \theta}$ (see Fig 2.1). For $\hat{u}_{\theta}^{h}\left(\lambda, t^{\prime \prime}\right)$ we have

Lemma 2.1. (a) $\hat{u}_{\theta}^{h}\left(\lambda, t^{\prime \prime}\right)$ is an entire function of $\lambda$.

(b) For any $\varepsilon>0$, there is a $C_{\varepsilon}>0$ such that

$$
\sup _{\left|t^{\prime \prime}\right| \leqq R}\left|\hat{u}_{\theta}^{h}\left(\lambda, t^{\prime \prime}\right)\right| \leqq C_{\varepsilon} \exp (\varepsilon|\lambda|) \quad \text { for } \lambda \text { with }|\arg \lambda+\theta|<\pi / 2 \text {. }
$$

(c) If $\sup _{\left|t^{\prime \prime}\right| \leqq R}\left|u^{h}\left(t_{0}, t^{\prime \prime}\right)\right| \leqq A \exp \left(\delta\left|t_{0}\right|^{-\gamma}\right) \quad(\gamma>0)$, then

(2.6) $\sup _{\left|t^{\prime \prime}\right| \leqq R}\left|\hat{u}_{\theta}^{h}\left(\lambda, t^{\prime \prime}\right)\right| \leqq B \exp \left(2\left(\delta|\lambda|^{\gamma}\right)^{1 /(1+\gamma)}\right) \quad$ for $\lambda$ with $|\arg \lambda+\theta|<\pi / 2$.

For the inversion formula we set

$$
u_{\theta}^{h}\left(z, z^{\prime \prime}\right)=\frac{1}{2 \pi i} \int_{\Lambda(\psi)} \exp \left(\lambda z_{0}\right) \hat{u}_{\theta}^{h}\left(\lambda, t^{\prime \prime}\right)(\log \lambda) d \lambda,
$$

where $|\theta+\psi|<\pi / 2$ and $\Lambda(\psi)$ is an infinite path starting at $\infty e^{i \psi}$, going around the origin once and ending at $\infty e^{i(\psi+2 \pi)}$ (see Fig 2.2).

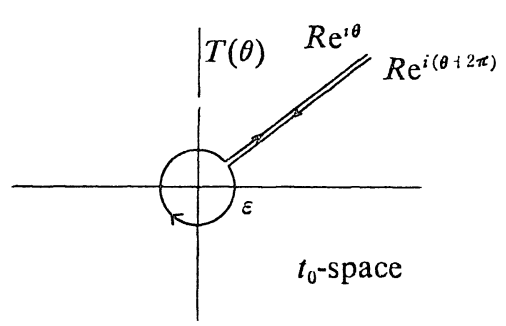

Fig. 2.1.

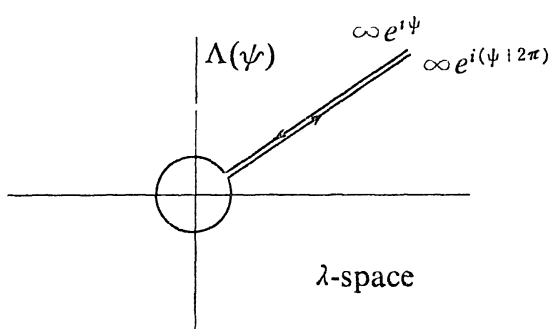

Fig. 2.2. 


\section{Lemma 2.2. It holds that}

$$
u_{\theta}^{h}\left(z_{0}, z^{\prime \prime}\right)=u^{h}\left(z_{0}, z^{\prime \prime}\right)+v_{\theta}^{h}\left(z_{0}, z^{\prime \prime}\right)
$$

for $z_{0}$ with $\theta<\arg z_{0}<\theta+2 \pi$, where $v_{\theta}^{h}\left(z_{0}, z^{\prime \prime}\right) \in \mathcal{O}\left\{\left|z_{0}\right|<R,\left|z^{\prime \prime}\right| \leqq R\right\}$.

The proofs of Lemma 2.1 and 2.2 are in $\S 6$.

(II) Kernel functions $w^{h}\left(z, t^{\prime \prime}, \lambda, \zeta\right)\left(0 \leqq h \leqq s_{m}-1\right)$. Each $w^{h}\left(z, t^{\prime \prime}, \lambda, \zeta\right)$ is determined so that it satisfies an equation. Let us derive the equation. In order to do so we derive operators $L\left(z, \lambda, \partial_{z}\right)$ and $\mathcal{L}\left(z, \lambda, \partial_{z}, \partial_{\zeta}\right)$ from $L\left(z, \partial_{z}\right)$. Firstly $L\left(z, \lambda, \partial_{z}\right)$ is defined as follows:

$$
\left\{\begin{array}{l}
L\left(z, \partial_{z}\right)\left\{\exp \left(\lambda z_{0}\right) K(z, \lambda)\right\}=\exp \left(\lambda z_{0}\right) L\left(z, \lambda, \partial_{z}\right) K(z, \lambda), \\
L\left(z, \lambda, \partial_{z}\right)=L\left(z, \partial_{0}+\lambda, \partial^{\prime}\right)=\sum_{k=0}^{m}\left\{\sum_{i=s_{k}}^{k} \lambda^{k-i} L_{k, i}\left(z, \partial_{z}\right)\right\}, \\
L_{k, i}\left(z, \partial_{z}\right)=\sum_{l \geqq s_{k}, l+j=i} A_{k, l}\left(z, \partial^{\prime}\right)\left({ }^{k-l}{ }_{j}\right)\left(\partial_{0}\right)^{j} .
\end{array}\right.
$$

Secondly we define $\mathcal{L}\left(z, \lambda, \partial_{z}, \partial_{\zeta}\right)$ from $L\left(z, \lambda, \partial_{z}\right)$. From Lemma 1.1 we have $k-i=(1-\alpha)\left(m-s_{m}\right)+\alpha(m-i)-(1-\alpha)\left(i-s_{k}\right)-\beta_{k}$. Hence by omitting $\lambda^{(1-\alpha)\left(m-s_{m}\right)}$ and replacing $\lambda^{\infty}$ by $\partial_{\zeta}$ in (2.9), we set

$$
\mathcal{L}\left(z, \lambda, \partial_{z}, \partial_{\zeta}\right)=\sum_{k=0}^{m}\left\{\sum_{i=s_{k}}^{k} \lambda^{-(1-\alpha)\left(i-s_{k}\right)-\beta_{k}}\left(\partial_{\zeta}\right)^{m-i} L_{k, i}\left(z, \partial_{z}\right)\right\}
$$

Thus we attain to the equation $w^{h}\left(z, t^{\prime \prime}, \lambda, \zeta\right)$ satisfies,

$$
\left\{\begin{array}{l}
\mathcal{L}\left(z, \lambda, \partial_{z}, \partial_{\zeta}\right) w^{h}\left(z, t^{\prime \prime}, \lambda, \zeta\right)=0 \\
\left.\left(\partial_{1}\right)^{l} w\left(z, t^{\prime \prime}, \lambda, \zeta\right)\right|_{z_{1}=0}=\delta_{l, h}(2 \pi i)^{-n} \zeta^{-1} \prod_{i=2}^{n}\left(t_{i}-z_{i}\right)^{-1},
\end{array}\right.
$$

for $0 \leqq l \leqq s_{m}-1$, where $|z| \leqq R^{\prime}$ and $R^{\prime}<R_{1} \leqq\left|t_{i}\right| \leqq R(i \geqq 2)$. We note that the initial values are singular at $\zeta=0$. For the existence of $w^{h}\left(z, \lambda, t^{\prime \prime}, \zeta\right)$ we have

Proposition 2.3. There is a solution $w^{h}\left(z, t^{\prime \prime}, \lambda, \zeta\right)$ of (2.11) which is multivalued holomorphic in

$$
\begin{aligned}
& Z=\left\{\left(z, t^{\prime \prime}, \lambda, \zeta\right) ;|z| \leqq r, R_{1} \leqq\left|t_{i}\right| \leqq R(i \geqq 2),|\lambda| \geqq \Lambda_{0},\right. \\
& \left.\quad A^{*}\left|z_{1}\right|<|\zeta|<B^{*}|\lambda|^{1-\alpha}\right\},
\end{aligned}
$$

where $r(r<R), \Lambda_{0}, A^{*}$ and $B^{*}$ are some positive constants, and $\left|w^{h}\left(z, t^{\prime \prime}, \lambda, \zeta\right)\right|$ $\leqq C\left(1+|\zeta|^{-1}+|\log \zeta|\right)$ holds.

The proof is given in $\S 5$.

(III) Integral representation. We can define by Proposition 2.3 


$$
K_{\theta}^{h}\left(z, t^{\prime \prime}, \lambda\right)=\int_{c(\theta)} \exp \left(-\lambda^{a} \zeta\right) w^{h}\left(z, t^{\prime \prime}, \lambda, \zeta\right) d \zeta,
$$

where $C(\theta)=C\left(d e^{i \theta} \lambda^{1-\alpha}\right)\left(0<d<B^{*}\right.$, where $B^{*}$ is in Proposition 2.3) is a path on the circle $|\zeta|=d|\lambda|^{1-\alpha}$ whose starting point is $d e^{i \theta} \lambda^{1-\alpha}$ and goes around once on it. For $K_{\theta}^{h}\left(z, t^{\prime \prime}, \lambda\right)$, we have

Proposition 2.4. $K_{\theta}^{h}\left(z, t^{\prime \prime}, \lambda\right)\left(0 \leqq h \leqq s_{m}-1\right)$ are single valued holomorphic functions with respect to $\lambda$ in $\Xi$,

$$
\Xi=\left\{\left(z, t^{\prime \prime}, \lambda\right) ;|z| \leqq r, R_{1} \leqq\left|t_{i}\right| \leqq R(i \geqq 2),|\lambda| \geqq \Lambda_{0}\right\},
$$

and satisfy

$$
\left\{\begin{array}{l}
L\left(z, \lambda, \partial_{z}\right) K_{\theta}^{h}\left(z, t^{\prime \prime}, \lambda\right)=\exp \left(-d e^{i \theta} \lambda\right) K^{\prime h}\left(z, t^{\prime \prime}, \lambda\right) \\
\left(\partial / \partial z_{1}\right)^{l} K_{\theta}^{h}\left(z, t^{\prime \prime}, \lambda\right)=\delta_{l, h}(2 \pi i)^{-n+1} \Pi_{i=2}^{n}\left(t_{i}-z_{i}\right)^{-1} \quad\left(0 \leqq l \leqq s_{m}-1\right)
\end{array}\right.
$$

where

$$
\left|K_{\theta}^{h}\left(z, t^{\prime \prime}, \lambda\right)\right| \leqq C|\lambda|^{2(1-\alpha)} \exp \left(c\left|z_{1}\right||\lambda|^{\alpha}\right)
$$

for $\lambda$ with $|\arg \lambda+\theta|<\pi / 2$, and $K^{\prime h}\left(z, t^{\prime \prime}, \lambda\right)$ is holomorphic in $\Xi$ and

$$
\left|K^{\prime h}\left(z, t^{\prime \prime}, \lambda\right)\right| \leqq A(1+|\lambda|)^{N} \quad \text { for some } N>0 .
$$

The proof is given in $\S 5$ except that $K_{\theta}^{h}\left(z, t^{\prime \prime}, \lambda\right)$ is single valued, which is proved in $\$ 9$.

Finally $\int_{T^{\prime \prime}} \cdots d t^{\prime \prime}$ means $\int_{\left|t_{2}\right|=R} \cdots d t_{2} \int_{\left|t_{3}\right|=R} \cdots d t_{3} \cdots \int_{\left|t_{n}\right|=R} \cdots d t_{n}$. So we have $(2 \pi i)^{-n+1} \int_{T^{\prime \prime}} f\left(t^{\prime \prime}\right) / \prod_{i=2}^{n}\left(t_{i}-z_{i}\right) d t^{\prime \prime}=f\left(z^{\prime \prime}\right)$ for a holomorphic function $f\left(z^{\prime \prime}\right)$.

Thus we attain to

Theorem 2.5. Assume (2.1)'. Then $u(z) \in \mathcal{O}\left(\tilde{\Omega}\left(\theta_{0}\right)\right)\left(\theta_{0}>\pi\right)$ satisfying $L$ $\left(z, \partial_{z}\right) u(z)=f(z) \in \mathcal{O}(\Omega)$ has an integral representation in $\tilde{U}(\theta, \theta+2 \pi)\left(-\theta_{0}<\theta\right.$ $\left.<\theta_{0}-2 \pi\right), U=\left\{z \in C^{n+1} ;|z| \leqq r\right\}(r<R)$, of the form

$$
u(z)=\sum_{h=0}^{s_{m}{ }^{-1}} u_{\theta}^{h}(z)+v_{\theta}(z),
$$

where $v_{\theta}(z) \in \mathcal{O}(U)$ and

$$
\begin{aligned}
& u_{\theta}^{h}(z)=\frac{1}{2 \pi i} \int_{\Lambda(\psi)} \exp \left(\lambda z_{0}\right) \log \lambda d \lambda \int_{T^{\prime \prime}} K_{\theta}^{h}\left(z, t^{\prime \prime}, \lambda\right) \hat{u}_{\theta}^{h}\left(\lambda, t^{\prime \prime}\right) d t^{\prime \prime} \\
& \quad(|\theta+\psi|<\pi / 2)
\end{aligned}
$$




\section{§. Construction of Kernel Function $w\left(z, t^{\prime \prime}, \lambda, \zeta\right)$-(I)}

Now we proceed to find a solution to the equation (2.11). We denote $w^{h}\left(z, t^{\prime \prime}, \lambda, \zeta\right)$ by $w\left(z, t^{\prime \prime}, \lambda, \zeta\right)$, omitting $h$. Let us write it again:

$$
\left\{\begin{array}{l}
\mathcal{L}\left(z, \lambda, \partial_{z}, \partial_{\zeta}\right) w\left(z, t^{\prime \prime}, \lambda, \zeta\right)=0 \\
\left.\left(\partial_{1}\right)^{l} w\left(z, t^{\prime \prime}, \lambda, \zeta\right)\right|_{z_{1}=0}=\delta_{l, h}(2 \pi i)^{-n} \zeta^{-1} \Pi_{i=2}^{n}\left(t_{i}-z_{i}\right)^{-1} \\
\quad \text { for } 0 \leqq l \leqq s_{m}-1,
\end{array}\right.
$$

where

$$
\mathcal{L}\left(z, \lambda, \partial_{z}, \partial_{\zeta}\right)=\sum_{k=0}^{m}\left\{\sum_{i=s_{k}}^{k} \lambda^{-(1-\alpha)\left(i-s_{k}\right)-\beta_{k}}\left(\partial_{\zeta}\right)^{m-i} L_{k, i}\left(z, \partial_{z}\right)\right\} .
$$

We construct $w\left(z, t^{\prime \prime}, \lambda, \zeta\right)$ under the condition $(2.1)^{\prime}$, that is,

$$
A_{m, s_{m}}\left(0, z^{\prime}, \hat{\xi}^{\prime}\right) \neq 0 \quad \text { for } \quad\left|z^{\prime}\right| \leqq R_{0}\left(R_{0}<R\right) .
$$

Firstly let us introduce auxilliary functions $\left\{f_{j}(\zeta)\right\}(j \in \mathbb{Z})$ used in [2],

$$
\left\{\begin{array}{l}
f_{j}(\zeta)=\frac{\zeta^{j}}{(2 \pi i) j !}\{\log \zeta-(1+1 / 2+\cdots+1 / j) \quad(j \geqq 1) \\
f_{0}(\zeta)=\frac{1}{2 \pi i} \log \zeta \\
f_{j}(\zeta)=(-1)^{j} \frac{(-j-1) !}{2 \pi i} \zeta^{j} \quad(j \leqq-1)
\end{array}\right.
$$

We note an important relation

$$
(d / d \zeta) f_{j}(\zeta)=f_{j-1}(\zeta)
$$

We try to find $w\left(z, t^{\prime \prime}, \lambda, \zeta\right)$ of the form

$$
\left\{\begin{array}{l}
w\left(z, t^{\prime \prime}, \lambda, \zeta\right)=\frac{1}{2 \pi i} \int_{\gamma} V\left(z, t^{\prime \prime}, \lambda, \zeta, \tau\right) d \tau, \\
V\left(z, t^{\prime \prime}, \lambda, \zeta, \tau\right)=\sum_{p=h-1}^{+\infty} v_{p}\left(z, t^{\prime \prime}, \lambda, \tau\right) f_{p}\left(\zeta+\tau z_{1}\right),
\end{array}\right.
$$

where $r$ is a closed path in $\tau$-space which will be determined later. Thus it becomes the main purpose to obtain equations which determine $v_{p}\left(z, t^{\prime \prime}, \lambda, \tau\right)$ $(p \geqq h-1)$. Let us give a lemma for calculations.

Lemma 3.1. There are operators $L_{k, i}^{j}\left(z, \partial_{z}\right)(0 \leqq j \leqq i)$ with ord. $L_{k, i}^{j}\left(z, \partial_{z}\right)$ $\leqq j$ and $L_{k, i}^{0}\left(z, \partial_{z}\right)=A_{k, i}\left(z, \hat{\xi}^{\prime}\right)$ such that

$$
\begin{aligned}
& \left(\partial_{\zeta}\right)^{m-i} L_{k, i}\left(z, \partial_{z}\right)\left\{v(z) f_{p}\left(\zeta+\tau z_{1}\right)\right\} \\
& \quad=\sum_{j=0}^{i}\left\{\tau^{i-j} L_{k, i}^{j}\left(z, \partial_{z}\right) v(z)\right\} f_{p-m+j}\left(\zeta+\tau z_{1}\right) .
\end{aligned}
$$

The proof is easy, so we omit it. Now we have from Lemma 3.1 


$$
\begin{aligned}
& \mathcal{L}\left(z, \lambda, \partial_{2}, \partial_{\zeta}\right) V\left(z, t^{\prime \prime}, \lambda, \zeta\right) \\
& \quad=\sum_{p=h-1-m}^{+\infty}\left\{\sum_{j=0}^{m} G_{j}\left(z, \lambda, \tau, \partial_{z}\right) v_{p+m-j}\left(z, t^{\prime \prime}, \lambda, \tau\right)\right\} f_{p}\left(\zeta+\tau z_{1}\right)
\end{aligned}
$$

where

$$
\left\{\begin{array}{l}
G_{0}\left(z, \lambda, \tau, \partial_{z}\right)=\sum_{k=0}^{m}\left\{\sum_{i=s_{k}}^{k} \lambda^{-(1-\alpha)\left(i-s_{k}\right)-\beta_{k}} \tau^{i} A_{k, i}\left(z, \hat{\xi}^{\prime}\right)\right\} \\
G_{j}\left(z, \lambda, \tau, \partial_{z}\right)=\sum_{k=j}^{m}\left\{\sum_{i=\max \left(s_{k}, j\right)}^{k} \lambda^{-(1-\alpha)\left(i-s_{k}\right)-\beta_{k}} \tau^{i-j} L_{k, i}^{j}\left(z, \partial_{z}\right)\right\}
\end{array}\right.
$$

We have ord. $G_{j}\left(z, \lambda, \tau, \partial_{z}\right) \leqq j$. Hence $G_{0}\left(z, \lambda, \tau, \partial_{z}\right)$ is a polynomial of $\tau$, so we denote it by $G_{0}(z, \lambda, \tau)$. Set

$$
g_{p}\left(z, t^{\prime \prime}, \lambda, \tau\right)=-\sum_{j=1}^{m} G_{j}\left(z, \lambda, \tau, \partial_{z}\right) v_{p-j}\left(z, t^{\prime \prime}, \lambda, \tau\right) .
$$

We have from (3.8)

$$
\begin{aligned}
& \mathcal{L}\left(z, \lambda, \partial_{z}, \partial_{\zeta}\right) V\left(z, t^{\prime \prime}, \lambda, \zeta\right) \\
& \quad=\sum_{p=h-1-m}^{+\infty}\left\{G_{0}(z, \lambda, \tau) v_{p+m}\left(z, t^{\prime \prime}, \lambda, \tau\right)-g_{p+m}\left(z, t^{\prime \prime}, \lambda, \tau\right)\right\} f_{p}\left(\zeta+\tau z_{1}\right)
\end{aligned}
$$

Hence we'll try to determine $v_{p}\left(z, t^{\prime \prime}, \lambda, \tau\right)(p \geqq h-1)$ by the following equations containing other unknown functions $h_{p}\left(z, t^{\prime \prime}, \lambda, \tau\right)(p \geqq h-1)$ :

$$
\begin{gathered}
G_{0}(z, \lambda, \tau) v_{h-1}\left(z, t^{\prime \prime}, \lambda, \tau\right)=h_{h-1}\left(z, t^{\prime \prime}, \lambda, \tau\right), \\
G_{0}(z, \lambda, \tau) v_{p}\left(z, t^{\prime \prime}, \lambda, \tau\right)=g_{p}\left(z, t^{\prime \prime}, \lambda, \tau\right)+h_{p}\left(z, t^{\prime \prime}, \lambda, \tau\right) .
\end{gathered}
$$

We'll define the path $r$ in (3.6) and solve the equations (3.12) ${ }_{p}$ in the next section. $\left\{h_{p}\left(z, t^{\prime \prime}, \lambda, \tau\right)\right\}$ are polynomials of $\tau$ with degree $\leqq s_{m}-1$ and are chosen so that $w\left(z, t^{\prime \prime}, \lambda, \zeta\right)$ satisfies the initial conditions in (3.1).

\section{$\S 4$. Construction of Kernel Function $w\left(z, t^{\prime \prime}, \lambda, \zeta\right)$-(II)}

In $\S 4$ we define the path $r,\left\{v_{p}\left(z, t^{\prime \prime}, \lambda, \tau\right)\right\}$ and $\left\{h_{p}\left(z, t^{\prime \prime}, \lambda, \tau\right)\right\}$. Firstly we define the path $r$ in (3.6). In order to do so, we need a lemma concerning the roots of $G_{0}(z, \lambda, \tau)=0$ (see (3.9)), which is an algebraic equation of $\tau$ :

Lemma 4.1. Assume (3.3). Then there are positive constants $a, b$ and $\Lambda_{0}$ such that if $|\lambda| \geqq \Lambda_{0}$, then $G_{0}(z, \lambda, \tau) \neq 0$ on $\left\{a \leqq|\tau| \leqq b|\lambda|^{1-\alpha}\right\}$ and there exist exactly $s_{m}$ roots of $G_{0}(z, \lambda, \tau)=0$ in $\{|\tau|<a\}$.

The proof of Lemma 4.1 is given in $\$ 6$. Lemma 4.1 means that there are exactly $s_{m}$-roots which are bounded as $|\lambda| \rightarrow+\infty$. The closed path $r$ in $\tau$ space is chosen so that it encloses all the bounded roots of $G_{0}(z, \lambda, \tau)=0$.

Now let us proceed to the determination of $V\left(z, t^{\prime \prime}, \lambda, \zeta, \tau\right)$ in (3.6), 


$$
V\left(z, t^{\prime \prime}, \lambda, \zeta, \tau\right)=\sum_{p=h-1}^{+\infty} v_{p}\left(z, t^{\prime \prime}, \lambda, \tau\right) f_{p}\left(\zeta+\tau z_{1}\right)
$$

We put $v_{p}\left(z, t^{\prime \prime}, \lambda, \tau\right)=h_{p}\left(z, t^{\prime \prime}, \lambda, \tau\right)=0$ for $p \leqq h-2$. Consider the initial conditions of $w\left(z, t^{\prime \prime}, \lambda, \tau\right)$ in (3.1). We have

$$
\left(\partial_{1}\right)^{l} V\left(z, t^{\prime \prime}, \lambda, \zeta, \tau\right)=\sum_{p=h-1}^{+\infty}\left\{\sum_{i=0}^{l}\left(\begin{array}{l}
l \\
i
\end{array}\right) v_{p-i}^{(i)}\left(z, t^{\prime \prime}, \lambda, \tau\right) \tau^{l-i}\right\} f_{p-l}\left(\zeta+\tau z_{1}\right),
$$
where $v^{(i)}\left(z, t^{\prime \prime}, \lambda, \tau\right)=\left(\partial_{1}\right)^{i} v\left(z, t^{\prime \prime}, \lambda, \tau\right)$. We define $C_{p, l}\left(z_{0}, z^{\prime \prime}, t^{\prime \prime}, \lambda\right)\left(0 \leqq l \leqq s_{m}\right.$ $-1)$ by

$$
\begin{aligned}
& C_{p, l}\left(z_{0}, z^{\prime \prime}, t^{\prime \prime}, \lambda\right)=\delta_{p, l-1} \delta_{l, h}(2 \pi i)^{-(n-1)} \Pi_{i=2}^{n}\left(t_{i}-z_{i}\right)^{-1} \\
& \quad-\frac{1}{2 \pi i}\left\{\int_{\gamma}\left(\sum_{i=1}^{l}\left(\begin{array}{l}
l \\
i
\end{array}\right) v_{p-i}^{(i)}\left(z, t^{\prime \prime}, \lambda, \tau\right) \tau^{l-i}\right) d \tau\right. \\
& \left.\quad+\int_{\gamma} \frac{g_{p}\left(z, t^{\prime \prime}, \lambda, \tau\right)}{G_{0}(z, \lambda, \tau)} \tau^{l} d \tau\right\}\left.\right|_{z_{1}=0} .
\end{aligned}
$$

We note that $C_{p, l}\left(z_{0}, z^{\prime \prime}, t^{\prime \prime}, \lambda\right)=0$ for $p \leqq h-2$. If $v_{p-i}\left(z, t^{\prime \prime}, \lambda, \tau\right)(i \geqq 1)$ are determined, since $g_{p}\left(z, t^{\prime \prime}, \lambda, \tau\right)$ (see (3.10)) contains only $v_{p-i}\left(z, t^{\prime \prime}, \lambda, \tau\right)(i \geqq 1)$, then $C_{p, l}\left(z_{0}, z^{\prime \prime}, t^{\prime \prime}, \lambda\right)$ are also done. By making use of $C_{p, l}\left(z_{0}, z^{\prime \prime}, t^{\prime \prime}, \lambda\right)$, we determine $h_{p}\left(z, t^{\prime \prime}, \lambda, \tau\right)$, which is a polynomial of $\tau$ with degree $\leqq s_{m}-1$ so that it satisfies

$$
\frac{1}{2 \pi i} \int_{\gamma} \frac{h_{p}\left(z, t^{\prime \prime}, \lambda, \tau\right)}{G_{0}(z, \lambda, \tau)} \tau^{l} d \tau=C_{p, l}\left(z_{0}, z^{\prime \prime}, t^{\prime \prime}, \lambda\right) \quad \text { for } \quad 0 \leqq l \leqq s_{m}-1 .
$$

It follows from Lemma 9.8 in $\S 9-$ II that $h_{p}\left(z, t^{\prime \prime}, \lambda, \tau\right)$ satisfying (4.4) uniquely exists. So we set

$$
v_{p}\left(z, t^{\prime \prime}, \lambda, \tau\right)=\left\{g_{p}\left(z, t^{\prime \prime}, \lambda, \tau\right)+h_{p}\left(z, t^{\prime \prime}, \lambda, \tau\right)\right\} / G_{0}(z, \lambda, \tau) .
$$

Thus $v_{p}\left(z, t^{\prime \prime}, \lambda, \tau\right)$ and $h_{p}\left(z, t^{\prime \prime}, \lambda, \tau\right)$ are successively determined. We notice that $v_{p}\left(z, t^{\prime \prime}, \lambda, \tau\right)(p \geqq h-1)$ have poles as functions of $\tau$ and the poles are the zeros of $G_{0}(z, \lambda, \tau)$.

Let us check that

$$
\begin{aligned}
w\left(z, t^{\prime \prime}, \lambda, \zeta\right) & =\frac{1}{2 \pi i} \int_{\gamma} V\left(z, t^{\prime \prime}, \lambda, \zeta, \tau\right) d \tau \\
& =\frac{1}{2 \pi i} \int_{\gamma} \sum_{p=h-1}^{+\infty} v_{p}\left(z, t^{\prime \prime}, \lambda, \tau\right) f_{p}\left(\zeta+\tau z_{1}\right) d \tau
\end{aligned}
$$

formally satisfies (3.1). The calculation which we perform below are justified after obtaining the estimates of $v_{p}\left(z, t^{\prime \prime}, \lambda, \tau\right)$ and the convergence of $V\left(z, t^{\prime \prime}, \lambda, \zeta, \tau\right)$. Assuming $|\zeta|>\left|\tau z_{1}\right|$ for $|z|<r$ and $\tau \in r, r$ being a small positive constant, we have 
(4.7)

$$
\begin{aligned}
\mathcal{L}\left(z, \lambda, \partial_{z}, \partial_{\zeta}\right) w\left(z, t^{\prime \prime}, \lambda, \zeta\right) \\
=\frac{1}{2 \pi i} \int_{\gamma} \sum_{p=h-1-m}^{+\infty}\left\{\sum_{j=0}^{m} G_{j}\left(z, \lambda, \tau, \partial_{z}\right) v_{p+m-j}\left(z, t^{\prime \prime}, \lambda, \tau\right)\right\} f_{p}\left(\zeta+\tau z_{1}\right) d \tau \\
=\frac{1}{2 \pi i} \int_{\gamma} \sum_{p=h-1-m}^{+\infty}\left\{G_{0}(z, \lambda, \tau) v_{p+m}\left(z, t^{\prime \prime}, \lambda, \tau\right)-g_{p+m}\left(z, t^{\prime \prime}, \lambda, \tau\right)\right\} \\
\\
\quad f_{p}\left(\zeta+\tau z_{1}\right) d \tau \\
=\frac{1}{2 \pi i} \int_{\gamma} \sum_{p=h-1-m}^{+\infty} h_{p+m}\left(z, t^{\prime \prime}, \lambda, \tau\right) f_{p}\left(\zeta+\tau z_{1}\right) d \tau=0 .
\end{aligned}
$$

Here we note that $\tau=-\zeta / z_{1}$, the singular point of $\log \left(\zeta+\tau z_{1}\right)$, is not in the inside of $\gamma$. As for the initial values, we have from (4.2)

$$
\begin{aligned}
& \left(\partial_{1}\right)^{l} w\left(z, t^{\prime \prime}, \lambda, \zeta\right) \\
& \quad=\frac{1}{2 \pi i} \int_{\gamma} \sum_{p=h-1}^{+\infty}\left\{\sum_{i=0}^{l}\left(\begin{array}{l}
l \\
i
\end{array}\right) v_{p-i}^{(i)}\left(z, t^{\prime \prime}, \lambda, \tau\right) \tau^{l-i}\right\} f_{p-l}\left(\zeta+\tau z_{1}\right) d \tau .
\end{aligned}
$$

It follows from (4.3) and (4.4) that

$$
\begin{aligned}
& \left.\left(\partial_{1}\right)^{l} w\left(z, t^{\prime \prime}, \lambda, \zeta\right)\right|_{z_{1}=0}=\frac{1}{2 \pi i} \sum_{p=h-1}^{+\infty}\left\{\int_{\gamma} v_{p}\left(z_{0}, 0, z^{\prime \prime}, \lambda, \zeta\right) \tau^{l} d \tau\right. \\
& -\int_{\gamma} \frac{g_{p}\left(z, t^{\prime \prime}, \lambda, \tau\right)+h_{p}\left(z, t^{\prime \prime}, \lambda, \tau\right)}{G_{0}(z, \lambda, \tau)} \tau^{l} d \tau+\delta_{p, l-1} \delta_{l, b}(2 \pi i)^{-n+1} \\
& \left.\quad \prod_{i=2}^{n}\left(t_{i}-z_{i}\right)^{-1}\right\} f_{p-l}(\zeta) \\
& =\delta_{l, h}(2 \pi i)^{-n} \zeta^{-1} \prod_{i=2}^{n}\left(t_{i}-z_{i}\right)^{-1} .
\end{aligned}
$$

Thus we conclude that $w\left(z, t^{\prime \prime}, \lambda, \zeta\right)$ satisfies formally the equation (3.1).

\section{§5. Construction of the Integral Representation}

In $\S 5$ we show the convergence of $V\left(z, t^{\prime \prime}, \lambda, \zeta, \tau\right)$, construct $w\left(z, t^{\prime \prime}, \lambda, \zeta\right)$ and $K_{\theta}\left(z, t^{\prime \prime}, \lambda\right)$, by integrating in $\tau$ and $\zeta$, and attain to the integral formula. In order to do so we need the estimates of $\left\{v_{p}\left(z, t^{\prime \prime}, \lambda, \tau\right) ; p \geqq h-1\right\}$. Before we give them, let us write again the set $\Xi((2.13))$, which will often appear in the sequel:

$$
\Xi=\left\{\left(z, t^{\prime \prime}, \lambda\right) ;|z| \leqq r, R_{1} \leqq\left|t_{i}\right| \leqq R(i \geqq 2),|\lambda| \geqq \Lambda_{0}\right\},
$$

where we'll make $r$ small and $\Lambda_{0}$ large if necessary.

(I) The convergence of $V\left(z, t^{\prime \prime}, \lambda, \zeta, \tau\right)$. We have

Proposition 5.1. For $v_{p}\left(z, t^{\prime \prime}, \lambda, \tau\right)(p \geqq h-1)$ the following estimates hold: there are $A$ and $B$ such that 


$$
\left|v_{p}\left(z, t^{\prime \prime} . \lambda, \tau\right)\right| \leqq A B^{p}|\tau|^{-p-2}(p+1) ! \quad \text { for } \quad\left(z, t^{\prime \prime}, \lambda, \tau\right) \in X
$$

where $X=\left\{\left(z, t^{\prime \prime}, \lambda, \tau\right) ;\left(z, t^{\prime \prime}, \lambda\right) \in \Xi, a \leqq|\tau| \leqq b|\lambda|^{1-\alpha}\right\}$ (see Lemma 4.1).

The proof of Proposition 5.1 is given with other estimates in $\S 9$. Now let us show the convergence of $V\left(z, t^{\prime \prime}, \lambda, \zeta, \tau\right)$. Set

$$
V_{1}\left(z, t^{\prime \prime}, \lambda, \zeta, \tau\right)=\sum_{p=0}^{+\infty}\left\{\left(\zeta+\tau z_{1}\right)^{p} / p !\right\} v_{p}\left(z, t^{\prime \prime}, \lambda, \tau\right)
$$

and

$$
V_{2}\left(z, t^{\prime \prime}, \lambda, \zeta, \tau\right)=\sum_{p=1}^{+\infty}\{(1+1 / 2+\cdots+1 / p) / p !\}\left(\zeta+\tau z_{1}\right)^{p} v_{p}\left(z, t^{\prime \prime}, \lambda, \tau\right) .
$$

Then by noting $h-1 \geqq-1$, we have

$$
\begin{aligned}
& V\left(z, t^{\prime \prime}, \lambda, \zeta, \tau\right)=\frac{1}{2 \pi i}\left\{V_{1}\left(z, t^{\prime \prime}, \lambda, \zeta, \tau\right) \log \left(\zeta+\tau z_{1}\right)\right. \\
& \left.\quad+V_{2}\left(z, t^{\prime \prime}, \lambda, \zeta, \tau\right)+\left(\zeta+\tau z_{1}\right)^{-1} v_{-1}\left(z, t^{\prime \prime}, \lambda, \tau\right)\right\} .
\end{aligned}
$$

We obatin

Lemma 5.2. $V_{i}\left(z, t^{\prime \prime}, \lambda, \zeta, \tau\right)(i=1,2)$ converge and $\left|V_{i}\left(z, t^{\prime \prime}, \lambda, \zeta, \tau\right)\right| \leqq$ $B|\tau|^{-2}$ in $\left\{\left(z, t^{\prime \prime}, \lambda, \zeta, \tau\right) ;\left(z, t^{\prime \prime}, \lambda, \tau\right) \in X,\left|\zeta+\tau z_{1}\right|<A|\tau|\right\}$.

Proof. From (5.2) we have $\left|\zeta+\tau z_{1}\right|^{p}\left|v_{p}\left(z, t^{\prime \prime}, \lambda, \tau\right)\right| / p ! \leqq A B^{p+1}\left|\zeta+\tau z_{1}\right|^{p} /|\tau|^{p+2}$. Hence if $B\left|\zeta+\tau z_{1}\right| /$ $|\tau|<1 / 2, V_{i}\left(z, t^{\prime \prime}, \lambda, \zeta, \tau\right)(i=1,2)$ are convergent and estimates hold.

Consequently

Proposition 5.3. $V\left(z, t^{\prime \prime}, \lambda, \zeta, \tau\right)$ is holomorphic in

$$
Y=\left\{\left(z, t^{\prime \prime}, \lambda, \zeta, \tau\right) ;\left(z, t^{\prime \prime}, \lambda, \tau\right) \in X, 0<\left|\zeta+\tau z_{1}\right|<A|\tau|\right\} .
$$

Corollary 5.4. $V\left(z, t^{\prime \prime}, \lambda, \zeta, \tau\right)$ is holomorphic and

$$
\left|V\left(z, t^{\prime \prime}, \lambda, \zeta, \tau\right)\right| \leqq A|\tau|^{-2}(1+|\log \zeta|)+B|\zeta|^{-1}
$$

in $\left\{\left(z, t^{\prime \prime}, \lambda, \zeta, \tau\right) ;\left(z, t^{\prime \prime}, \lambda, \tau\right) \in X,\left(A-\left|z_{1}\right|\right)|\tau|>|\zeta|>2\left|\tau z_{1}\right|\right\}$.

Proof. We have $A|\tau|>|\zeta|+\left|\tau z_{1}\right|>\left|\zeta+\tau z_{1}\right|>|\zeta|-\left|\tau z_{1}\right|>0$ and $\left|\tau z_{1} / \zeta\right|<1 / 2$ in the domain. So $\left|\zeta+\tau z_{1}\right|^{-1} \leqq 2 /|\zeta|$ and $\left|\log \left(\zeta+\tau z_{1}\right)\right| \leqq C+$ $|\log \zeta|$. The assertion follows easily.

(II) The construction of $w\left(z, t^{\prime \prime}, \lambda, \zeta\right)$ and $K_{\theta}\left(z, t^{\prime \prime}, \lambda\right)$. We perform integrating in $\tau$. We denote by $r(c)$ the path in $\tau$-space which starts at $\tau=c$, goes around once on $|\tau|=|c|$ and ends at $\tau=c \exp (2 \pi i)$. Set $\gamma_{1}=r(c), a \leqq$ 
$|c| \leqq b|\lambda|^{1-\infty}$ (see Proposition 5.1), and define

$$
w\left(z, t^{\prime \prime}, \lambda, \zeta\right)=\int_{\gamma_{1}} V\left(z, t^{\prime \prime}, \lambda, \zeta, \tau\right) d \tau .
$$

We can give the proof of Proposition 2.3 by Corollary 5.4.

Proof of Proposition 2.3. Suppose $|\tau|\left(A-\left|z_{1}\right|\right)>|\zeta|>2\left|\tau z_{1}\right|$ on $|\tau|=$ $|c|$. Then we have $\left|\tau z_{1} / \zeta\right|<1 / 2,\left|\zeta+\tau z_{1}\right| \leqq 2 /|\zeta|$ and $\left|\log \left(\zeta+\tau z_{1}\right)\right| \leqq C+$ $|\log \zeta|$ on $|\tau|=|c|$, and $w\left(z_{1}, \zeta\right)=w\left(z, t^{\prime \prime}, \lambda, \zeta\right)$ is holomorphic in $\left\{\left(z_{1}, \zeta\right) ;\left|z_{1}\right|\right.$ $\left.\leqq r,|c|\left(A-\left|z_{1}\right|\right)>|\zeta|>2\left|c z_{1}\right|\right\}$. Changing $c$ in $r_{1}=\gamma(c)\left(a \leqq|c| \leqq b|\lambda|^{1-\alpha}\right)$, we conclude that $w\left(z_{1}, \zeta\right)$ is holomorphic in $\left\{\left(z_{1}, \zeta\right) ; 2 a\left|z_{1}\right|<|\zeta|<b|\lambda|^{1-\infty}\right.$ $\left.\left(A-\left|z_{1}\right|\right),\left|z_{1}\right| \leqq \min (r, A / 3)\right\}$ and $\left|w\left(z_{1}, \zeta\right)\right| \leqq A\left(C+|\zeta|^{-1}+|\log \zeta|\right)$. This implies the assertion of Proposition 2.3.

Next we integrate $w\left(z, t^{\prime \prime}, \lambda, \zeta\right)$ in $\zeta$ and construct $K_{\theta}\left(z, t^{\prime \prime}, \lambda\right)$. Let us recall the path $C(\theta)$ in $\zeta$-space defined in $\S 2$-III. It is a path whose stating point is $d e^{i \theta} \lambda^{1-\alpha}\left(0<d<B^{*}\right)$ and goes around once on $|\zeta|=d|\lambda|^{1-\alpha}$.

Let us show some part of Proposition 2.4 about $K_{\theta}\left(z, t^{\prime \prime}, \lambda\right)$.

Proof of Proposition 2.4-(I). From Proposition 2.3 we can define

$$
K_{\theta}\left(z, t^{\prime \prime}, \lambda\right)=\int_{C(\theta)} \exp \left(-\lambda^{\alpha} \zeta\right) w\left(z, t^{\prime \prime}, \lambda, \zeta\right) d \zeta .
$$

Let us deform $C(\theta)$ to the path $C$ which starts at $d e^{i \theta} \lambda^{1-\alpha}$, goes to $c_{1} e^{i \theta}\left(c_{1}>\right.$ $\left.A^{*}\left|z_{1}\right|\right)$, goes around once on $|\zeta|=c_{1}$ and goes from $c_{1} e^{i(\theta+2 \pi)}$ to $d e^{i(\theta+2 \pi)} \lambda^{1-\alpha}$, $A^{*}$ being the same as in Proposition 2.3.

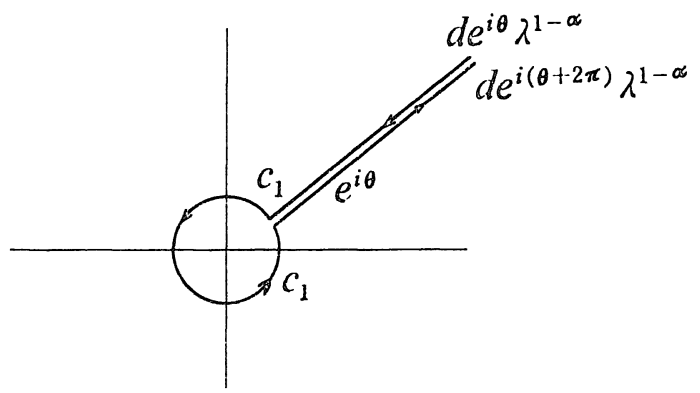

Fig. 5.1.

Thus we get, if $|\arg \lambda+\theta|<\pi / 2$,

$$
\left|K_{\theta}\left(z, t^{\prime \prime}, \lambda\right)\right| \leqq A|\lambda|^{2(1-\alpha)} \exp \left(c\left|z_{1} \lambda^{\infty}\right|\right) .
$$

It follows from the method of construction of $K_{\theta}\left(z, t^{\prime \prime}, \lambda\right)$ and integration by 
parts that

(5.9) $L\left(z, \lambda, \partial_{z}\right) K_{\theta}\left(z, t^{\prime \prime}, \lambda\right)$

$$
\begin{aligned}
& =\int_{C(\theta)} \exp \left(-\lambda^{\omega} \zeta\right) \mathcal{L}\left(z, \lambda, \partial_{z}, \partial_{\zeta}\right) w\left(z, t^{\prime \prime}, \lambda, \zeta\right) d \zeta+\exp \left(-d e^{i \theta} \lambda\right) K^{\prime}\left(z, t^{\prime \prime}, \lambda\right) \\
& =\exp \left(-d e^{i \theta} \lambda\right) K^{\prime}\left(z, t^{\prime \prime}, \lambda\right)
\end{aligned}
$$

where $\left|K^{\prime}\left(z, t^{\prime \prime}, \lambda\right)\right| \leqq A(1+|\lambda|)^{N}$ for some $N>0$, and

$$
\begin{aligned}
\left.\left(\partial_{1}\right)^{l} K_{\theta}\left(z, t^{\prime \prime}, \lambda\right)\right|_{z_{1}=0} & =\left.\int_{C(\theta)} \exp \left(-\lambda^{\alpha} \zeta\right)\left(\partial_{1}\right)^{l} w\left(z, t^{\prime \prime}, \lambda, \zeta\right) d \zeta\right|_{z_{1}=0} \\
& =\delta_{l, h}(2 \pi i)^{-n} \prod_{i=2}^{n}\left(t_{i}-z_{i}\right)^{-1} \int_{C(\theta)} \exp \left(-\lambda^{\alpha} \zeta\right) \zeta^{-1} d \zeta \\
& =\delta_{l, h}(2 \pi i)^{-(n-1)} \prod_{i=2}^{n}\left(t_{i}-z_{i}\right)^{-1}
\end{aligned}
$$

The proof of Proposition 2.4 is not yet completed. The rest of it is in §9-III.

(III) The integral representation. $K_{\theta}\left(z, t^{\prime \prime}, \lambda\right)$ is determined in (II). Hereafter we write suffix $h$ again, for example, $K_{\theta}^{h}\left(z, t^{\prime \prime}, \lambda\right), \hat{u}_{\theta}^{h}(z)$ etc.. We set

$$
u_{\theta}^{h}(z)=\frac{1}{2 \pi i} \int_{\Lambda(\psi)} \exp \left(\lambda z_{0}\right) \log \lambda d \lambda \int_{T^{\prime \prime}} K_{\theta}^{h}\left(z, t^{\prime \prime}, \lambda\right) \hat{u}_{\theta}^{h}\left(\lambda, t^{\prime \prime}\right) d t^{\prime \prime},
$$

where $|\psi+\theta|<\pi / 2$ and $0 \leqq h \leqq s_{m}-1$. Set $u_{\theta}(z)=\sum_{h=0}^{s_{m}-1} u_{\theta}^{h}(z)$. Then we have to show that $u_{\theta}(z)$ is a desired formula of the solution $u(z)$. We have

$$
\begin{aligned}
& L\left(z, \partial_{z}\right) u_{\theta}^{h}(z) \\
& \quad=\frac{1}{2 \pi i} \int_{\Lambda(\psi)} \exp \left(\lambda z_{0}\right) \log \lambda d \lambda \int_{T^{\prime \prime}} L\left(z, \lambda, \partial_{z}\right) K_{\theta}^{h}\left(z, t^{\prime \prime}, \lambda\right) \hat{u}_{\theta}^{h}\left(\lambda, t^{\prime \prime}\right) d t^{\prime \prime} \\
& \quad=\frac{1}{2 \pi i} \int_{\Lambda(\psi)} \exp \left(\lambda\left(z_{0}-d e^{i \theta}\right)\right) \log \lambda d \lambda \int_{T^{\prime \prime}} K^{\prime h}\left(z, t^{\prime \prime}, \lambda\right) \hat{u}_{\theta}^{h}\left(\lambda, t^{\prime \prime}\right) d t^{\prime \prime} \\
& \quad=f_{\theta}^{h}(z) .
\end{aligned}
$$

It is obvious that $f_{\theta}^{h}(z)$ is holomorphic in $\left\{\left|z_{0}\right|<d\right\} \cap\{|z|<r\}$. We have for the initial values, by Proposition 2.4,

(5.12) $\left.\left(\partial_{1}\right)^{l} u_{\theta}^{h}(z)\right|_{z_{1}=0}$

$$
\begin{aligned}
& =\left.(2 \pi i)^{-(n-1)} \int_{\Lambda(\psi)} \exp \left(\lambda z_{0}\right) \log \lambda d \lambda \int_{T^{\prime \prime}}\left(\partial_{1}\right)^{l} K_{\theta}^{h}\left(z, t^{\prime \prime}, \lambda\right)\right|_{z_{1}=0} \hat{u}_{\theta}^{h}\left(\lambda, t^{\prime \prime}\right) d t^{\prime \prime} \\
& =\delta_{l, h}(2 \pi i)^{-n+1} \int_{\Lambda(\psi)} \exp \left(\lambda z_{0}\right) \log \lambda d \lambda \int_{T^{\prime \prime}}\left\{\Pi_{i=2}^{n}\left(t_{i}-z_{i}\right)^{-1}\right\} \hat{u}_{\theta}^{h}\left(\lambda, t^{\prime \prime}\right) d t^{\prime \prime} \\
& =\frac{\delta_{l, h}}{2 \pi i} \int_{\Lambda(\psi)} \exp \left(\lambda z_{0}\right) \hat{u}_{\theta}^{h}\left(\lambda, z^{\prime \prime}\right) \log \lambda d \lambda .
\end{aligned}
$$


By Lemma 2.2 we have

$$
\left(\partial_{1}\right)^{b} u_{\theta}^{h}\left(z_{0}, 0, z^{\prime \prime}\right)=\delta_{l, h}\left\{\left(\partial_{1}\right)^{h} u\left(z_{0}, 0, z^{\prime \prime}\right)+v_{\theta}^{h}\left(z_{0}, z^{\prime \prime}\right)\right\},
$$

where $v_{\theta}^{h}\left(z_{0}, z^{\prime \prime}\right)$ is holomorphic at $z_{0}=z^{\prime \prime}=0$. Therefore we have

$$
\left\{\begin{array}{l}
L\left(z, \partial_{z}\right)\left(u(z)-u_{\theta}(z)\right)=f(z)-f_{\theta}(z), f_{\theta}(z)=\sum_{h=0}^{s_{m}-1} f_{\theta}^{h}(z), \\
\left.\left(\partial_{1}\right)^{l}\left(u(z)-u_{\theta}(z)\right)\right|_{z_{1}=0}=v_{\theta}^{l}\left(z_{0}, z^{\prime \prime}\right) \text { for } 0 \leqq l \leqq s_{m}-1,
\end{array}\right.
$$

where the functions in the right hand side of (5.14) are holomorphic at $z=0$ (or $z_{0}=z^{\prime \prime}=0$ ). Therefore by the uniqueness of the Goursat's problem means that $u(z)-u_{\theta}(z)$ is holomorphic at $z=0$ (see $\S 8$ in [6]). So $u_{\theta}(z)$ is a desired integral representation of $u(z)$.

\section{§ 6. Miscellaneous Results-(I)}

In $\S 6$ we summerize what we need. Some of it was used in the previous sections and others will be used in the later sections to show estimates and to deform integration paths. This section is divided into 4 parts. They are properties of $\left\{\beta_{k}^{i}\right\}$, zeros of $G_{0}(z, \lambda, \tau)$, sectors $S_{i}(1 \leqq i \leqq p-1)$ and proofs of Lemmas 2.1 and 2.2.

(I) Properties of $\left\{\beta_{k}^{i}\right\}$. We investigate $\left\{\beta_{k}^{i}\right\}$ defined in Lemma 1.1 in $\S 1$. We have set $\alpha=\alpha_{1}$ and $\beta_{k}=\beta_{k}^{1}$. Firstly we prove Lemma 1.1.

Proof of Lemma 1.1. If $k=k_{i-1}$, then we have $\beta_{k}^{i}=0$. Suppose $k \neq k_{i-1}$. Since $\Sigma$ is the lower convex part of the boundary of $\hat{A}$, there are $h_{k}^{i} \in \boldsymbol{Q}$, such that

$$
\left(s_{k_{i-1}}-s_{k}\right) /\left(k_{i-1}-k\right)+h_{k}^{i}=\sigma_{i}=\left(1-\alpha_{i}\right)^{-1},
$$

where $h_{k}^{i} \geqq 0$ if $k<k_{i-1}$, and $h_{k}^{i}<0$ if $k>k_{i-1}$. We have (1.5), by putting $\beta_{k}^{i}=$ $h_{k}^{i}\left(1-\alpha_{i}\right)\left(k_{i-1}-k\right)$, and $\beta_{k}^{i}=0$ if and only if $\left(k, s_{k}\right) \in \sum(i)$.

We further have

Proposition 6.1. The followings hold:

(1) $\beta_{k}+\left(\alpha-\alpha_{i}\right) s_{k}=\beta_{k_{i-1}}+\left(\alpha-\alpha_{i}\right) s_{k_{i-1}}+\beta_{k}^{i}$.

(2) For $\left(k, s_{k}\right) \in \Sigma(i), \beta_{k}+\left(\alpha-\alpha_{i}\right) s_{k}=\beta_{k_{i-1}}+\left(\alpha-\alpha_{i}\right) s_{k_{i-1}}$, in particular $\beta_{k_{i}}+$ $\left(\alpha-\alpha_{i}\right) s_{k_{i}}=\beta_{k_{i-1}}+\left(\alpha-\alpha_{i}\right) s_{k_{i-1}}$.

Proof. (1) It follows from Lemma 1.1 that $\left(s_{m}-s_{k}\right)(1-\alpha)+\beta_{k}=m-k$, $\left(s_{m}-s_{k_{i-1}}\right)(1-\alpha)+\beta_{k_{i-1}}=m-k_{i-1}$ and $\left(s_{k_{i-1}}-s_{k}\right)\left(1-\alpha_{i}\right)+\beta_{k}^{i}=k_{i-1}-k$. We obtain (1) from these equalities. (2) For $\left(k, s_{k}\right) \in \Sigma(i), \beta_{k}^{i}=0$. So from (1) 
we have the first equality and, by putting $k=k_{i}$, we get the second.

(II) Roots of $G_{0}(z, \lambda, \tau)=0$. We study the roots of $G_{0}(z, \lambda, \tau)=0$ (see (3.9)), which is an algebraic equation of $\tau$. Set

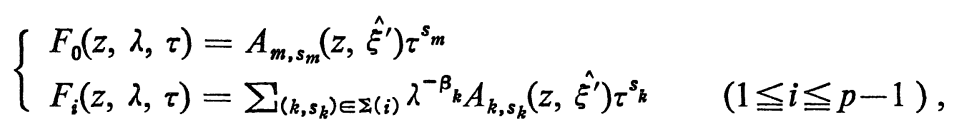

where $\hat{\xi}^{\prime}=(1,0, \cdots, 0)$. We give the condition on $G_{0}(z, \lambda, \tau)$

$$
\left|A_{k_{i}, s_{k_{i}}}\left(z, \hat{\xi^{\prime}}\right)\right| \geqq c_{0}>0 \quad \text { for } \quad|z| \leqq R_{0} .
$$

Some of $(6.3)$-i $(0 \leqq i \leqq p-1)$ will be assumed in the following lemmas and propositions.

Lemma 6.2. Assume (6.3)-i. Then there are positive constants $a_{i+1}, b_{i}$ and $\Lambda_{0}$ such that if $|\lambda| \geqq \Lambda_{0}$, then

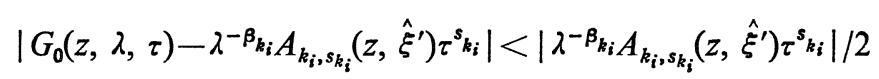

on $\left\{\tau ;|\tau|=b_{i}|\lambda|^{\alpha_{i}-\alpha}\right\} \cup\left\{\tau ;|\tau|=a_{i+1}|\lambda|^{\alpha_{i+1}{ }^{-\alpha}}\right\}$.

Proof. Let $|\tau|=c|\lambda|^{\alpha_{i}{ }^{-\alpha}}$. Then from Proposition 6.1 for each term in $G_{0}(z, \lambda, \tau)$

$$
\begin{aligned}
& \left|\lambda^{-\beta_{k}-(1-\alpha)\left(l-s_{k}\right)} \tau^{l} A_{k, l}\left(z, \hat{\xi}^{\prime}\right)\right|=\left|c^{l} \lambda^{-p} A_{k, l}\left(z, \hat{\xi}^{\prime}\right)\right|, \\
& p=\beta_{k_{i}}+\left(\alpha-\alpha_{i}\right) s_{k_{i}}+\beta_{k}^{i}+\left(1-\alpha_{i}\right)\left(l-s_{k}\right) .
\end{aligned}
$$

If $l=s_{k}$ and $\left(k, s_{k}\right) \in \sum(i)$, then $p=\beta_{k_{i}}+\left(\alpha-\alpha_{i}\right) s_{k_{i}}$. Hence there is a small $c>0$ such that

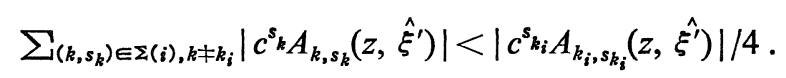

Fix $c>0$. For each term in $\left\{G_{0}(z, \lambda, \tau)-F_{i}(z, \lambda, \tau)\right\}, \beta_{k}^{i}>0$ or $l>s_{k}$. So this means $p>\left(\alpha-\alpha_{i}\right) s_{k_{i}}+\beta_{k_{i}}$. Therefore there is a large $\Lambda_{0}$ such that for $|\lambda| \geqq \Lambda_{0}$ and on $\left\{|\tau|=c|\lambda|^{\alpha_{i}-\alpha}\right\}$,

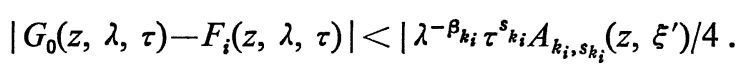

Thus we have (6.4) on $\left\{|\tau|=\left.b_{i}|\lambda|\right|^{\alpha_{i} i^{-\alpha}}\right\}\left(b_{i}=c\right)$. Next let $|\tau|=c|\lambda|^{\alpha_{i+1}{ }^{-\alpha}}$, we have

$$
\left|\lambda^{(1-\alpha)\left(l-s_{k}\right)-\beta_{k}} \tau^{l} A_{k, l}\left(z, \hat{\xi}^{\prime}\right)\right|=c^{l}\left|\lambda^{-q} A_{k, l}\left(z, \hat{\xi}^{\prime}\right)\right|,
$$

where $q=\beta_{k_{i}}+\left(\alpha-\alpha_{i+1}\right) s_{k_{i}}+\beta_{k}^{i}+\left(1-\alpha_{i+1}\right)\left(l-s_{k}\right)$. Hence for a large $c>0$ we have 


$$
\sum_{\left(k, s_{k}\right) \in \Sigma(i+1), k \neq k_{i}} c^{s_{k}}\left|A_{k, s_{k}}\left(z, \xi^{\prime}\right)\right|<c^{s_{k_{i}}}\left|A_{k_{i}, s_{k_{i}}}\left(z, \hat{\xi}^{\prime}\right)\right| / 4
$$

Fix $c>0$. Then in the same way as above there exists $a A_{0}$ such that for $|\lambda| \geqq \Lambda_{0}$

$$
\left|G_{0}(z, \lambda, \tau)-F_{i+1}(z, \lambda, \tau)\right|<\left|\tau^{s_{k_{i}}} A_{k_{i}, s_{k_{i}}}\left(z, \hat{\xi}^{\prime}\right)\right| / 4 .
$$

Thus on $\left\{|\tau|=a_{i+1}|\lambda|^{\alpha_{i+1}{ }^{-\alpha}}\right\}\left(|\lambda| \geqq \Lambda_{0}, a_{i+1}=c\right)$, we have (6.4). This completes the proof.

Now we can show Lemma 4.1.

Proof of Lemma 4.1. We have from Lemma 6.2

$$
\left|G_{0}(z, \lambda, \tau)-A_{m, s_{m}}\left(z, \hat{\xi}^{\prime}\right) \tau^{s_{m}}\right|<\left|A_{m, s_{m}}\left(z, \hat{\xi}^{\prime}\right) \tau^{s_{m}}\right|
$$

on $\left\{|\tau|=b_{0}|\lambda|^{1-\alpha}\right\} \cup\left\{|\tau|=a_{1}\right\}$. Hence it follows from the Rouche's Theorem in the theory of functions of one complex variable that there are $s_{m}$ roots of $G_{0}(z, \lambda, \tau)=0$ in $\left\{\tau ;|\tau|<a_{1}\right\}$ and no zeros in $\left\{a_{1} \leqq|\tau| \leqq b_{0}|\lambda|^{1-\alpha}\right\}$.

Secondly we study the roots of $G_{0}(z, \lambda, \tau)=0$ more precisely. We have

Lemma 6.3. Assume (6.3)-( $i-1)$ and (6.3)- $i \quad(i \neq 0)$. The equation $F_{i}(z, \lambda, \tau)=0$ has $\left(s_{k_{i-1}}-s_{k_{i}}\right)$ non zero roots $\left\{\tau_{i, j}(z) \lambda^{\alpha}{ }^{\alpha}{ }^{-\alpha} ; 1 \leqq j \leqq s_{k_{i-1}}-s_{k_{i}}\right\}$ and other roots are zero.

Proof. Set $\tau=\eta \lambda^{\alpha i^{-\alpha}}$. Then, by Proposition 6.1-(2),

$$
F_{i}\left(\eta \lambda^{\alpha i^{-\alpha}}\right)=\lambda^{-p} \sum_{\left(k, s_{k}\right) \in \Sigma(i)}\left(\eta^{s}{ }^{s} A_{k, s_{k}}\left(z, \hat{\xi}^{\prime}\right)\right),
$$

where $p=\beta_{k_{i}}+\left(\alpha-\alpha_{i}\right) s_{k_{i}}$. Thus $F_{i}(z, \lambda, \tau)=0$ has $\left(s_{k_{i-1}}-s_{k_{i}}\right)$ non zero roots $\left\{\tilde{\tau}_{i, j}(z) \lambda^{\alpha}{ }^{\alpha}{ }^{-\alpha} ; 1 \leqq j \leqq s_{k_{i-1}}-s_{k_{i}}\right\}$ and other roots are zero.

We set

$$
\stackrel{\circ}{N}_{i}=\left\{\circ_{i, j}(z) ;|z| \leqq R_{0}, 1 \leqq j \leqq s_{k_{i-1}}-s_{k_{i}}\right\},
$$

Proposition 6.4. Assume (6.3)-(i-1) and (6.3)- $i(i \neq 0)$. The equation $G_{0}(z, \lambda, \tau)=0$ has $\left(s_{k_{i-1}}-s_{k_{i}}\right)$ non zero roots $\left\{\tau_{i, j}(z, \lambda) ; 1 \leqq j \leqq s_{k_{i-1}}-s_{k_{i}}\right\}$ such that for $|\lambda| \geqq \Lambda_{0}$

$$
\left|\tau_{i, j}(z, \lambda)-\stackrel{\circ}{\tau}_{i, j}(z) \lambda^{\alpha i_{i}-\infty}\right| \leqq A|\lambda|^{-\rho+\alpha_{i}-\alpha},
$$

$\Lambda_{0}, A$ and $\rho$ being positive constants.

Proof. We choose $\rho>0$ so that $0<\rho\left(s_{k_{i-1}}-s_{k_{i}}\right)<d, d=\min \left\{1-\alpha_{i}, \beta_{k}^{i}\right.$ $\left.\left(k \notin \sum(i)\right)\right\}$. Put $D_{\eta}(i)=\left\{\eta ; \operatorname{dis}\left(\eta, \stackrel{\circ}{N}_{i}\right)=|\lambda|^{-\rho}\right\}$, dis $(\eta, K)$ being the distance 
from the point $\eta$ to the set $K$. For $\eta \in D_{\eta}(i)$, there is a $C>0$ such that

$$
\left|F_{i}\left(\lambda^{\alpha i_{i}-\alpha} \eta\right)\right| \geqq C|\lambda|^{-\beta_{k_{i}}-\left(\alpha-\alpha_{i}\right) s_{k_{i}}-\rho\left(s_{k_{i-1}}-s_{k_{i}}\right)} .
$$

We have from (6.5), for $\eta \in D_{\eta}(i)$

$$
\begin{aligned}
& \left|G_{0}\left(z, \lambda, \eta \lambda^{\alpha_{i}-\alpha}\right)-F_{i}\left(z, \lambda, \eta \lambda^{\alpha} i^{-\alpha}\right)\right| \\
& \leqq C|\lambda|^{-\beta_{k_{i}}+\left(\alpha_{i}-\alpha\right) s_{k_{i}}}\left(\sum_{l>s_{k} \text { or }\left(k, s_{k}\right) \oplus \Sigma(i)}|\lambda|^{\left.-\left(1-\alpha_{i}\right)\left(l-s_{k}\right)-\beta_{k}^{i}\right)}\right. \\
& \leqq C|\lambda|^{-\beta_{k_{i}}+\left(\alpha_{i}-\alpha\right) s_{k_{i}}-d} \text {. }
\end{aligned}
$$

Thus it holds for a large $\Lambda_{0}$ that if $\eta \in D_{\eta}(i)$ and $\left.|\lambda| \geqq \Lambda_{0},\left|F_{i}(z, \lambda, \tau)\right| / 2\right\rangle$ $\left|G_{0}(z, \lambda, \tau)-F_{i}(z, \lambda, \tau)\right|$. Therefore $G_{0}(z, \lambda, \tau)=0$ has $\left(s_{k_{i-1}}-s_{k_{i}}\right)$ roots in the inside of $\lambda^{\left({ }^{(\alpha}{ }_{i}-\alpha\right)} D_{\eta}(i)$ by the Rouche's Theorem.

Hereafter assume $p>1,(6.3)-i$ for all $0 \leqq i \leqq p-1$ and $s_{k_{p-1}}=0$. Set

$$
N_{i}(z, \lambda)=\left\{\tau_{i, j}(z, \lambda) \lambda^{\alpha-\alpha_{i}} ; 1 \leqq j \leqq s_{k_{i-1}}-s_{k_{i}}\right\}
$$

and

$$
K_{i}(\delta)=\cup_{j=1}^{s_{k_{i-1}}-s_{k_{i}}}\left\{\tau ;\left|\tau-\dot{\tau}_{i, j}(0)\right| \leqq \delta\right\} .
$$

We choose small $\delta, R_{0}>0$ and a large $\Lambda_{0}$ so that if $|z| \leqq R_{0}$ and $|\lambda| \geqq \Lambda_{0}$,

$$
K_{i}(\delta) \supset K_{i}(\delta / 2) \supset N_{i}(z, \lambda), K_{i}(\delta) \subset\left\{\tau ; b_{i}<|\tau|<a_{i}\right\}(1 \leqq i \leqq p-1),
$$

$a_{i}$ and $b_{i}$ being those in Lemma 6.2. Define the sets for $1 \leqq i \leqq p-1$

$$
\tau(i)=\left\{\tau ; b_{i}|\lambda|^{\alpha} i^{-\alpha} \leqq|\tau| \leqq b_{i-1}|\lambda|^{\alpha}{ }^{i_{-1}-\alpha}, \tau \notin \lambda^{\alpha} i^{-\alpha} K_{i}(\delta)\right\} .
$$

Then we have

Proposition 6.5. Let $N(z, \lambda)$ be the set of all bounded roots of $G_{0}(z, \lambda, \tau)=0$ as $\lambda \rightarrow \infty$. Then $N(z, \lambda) \subset \cup_{i=1}^{p-1} \lambda^{\alpha} i^{-\alpha} K_{i}(\delta / 2)$.

We have

Proposition 6.6. For $\tau \in \tau(i)$

$$
\left|G_{0}(z, \lambda, \tau)\right| \geqq C|\lambda|^{-\beta_{k_{i-1}}|\tau|^{s_{k_{i-1}}}} .
$$

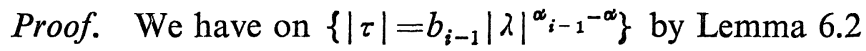

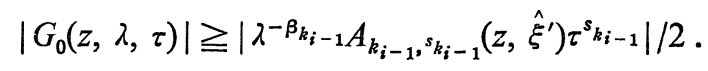

Similarly on $\left\{|\tau|=b_{i}|\lambda|^{\alpha_{i}-\alpha}\right\}$ or $\tau \in \lambda^{\alpha_{i}-\alpha} \partial K_{i}(\delta)$ we have

$$
\left|G_{0}(z, \lambda, \tau)\right| \geqq C|\lambda|^{-p} \geqq C|\lambda|^{-\beta_{k_{i-1}}}|\tau|^{s_{k_{i-1}}},
$$


where $p=\beta_{k_{i}}+\left(\alpha-\alpha_{i}\right) s_{k_{i}}=\beta_{k_{i-1}}+\left(\alpha-\alpha_{i}\right) s_{k_{i-1}}$ by Lemma 6.1-(2). Thus applying the maximal principle of holomorphic functions to $\tau^{s_{k_{i-1}}} G_{0}(z, \lambda, \tau)^{-1}$, we have $\left|\tau^{s_{k_{i-1}} G_{0}}(z, \lambda, \tau)^{-1}\right| \leqq C\left|\lambda^{\beta_{k_{i-1}}}\right|$. This means (6.12).

Proposition 6.7. For $\tau \in \tau(i)$ and $l \geqq s_{k}$ there is an $A$ such that

$$
\frac{\left|\lambda-(1-\alpha)\left(l-s_{k}\right)-\beta_{k} \tau^{l}\right|}{\left|G_{0}(z, \lambda, \tau)\right|} \leqq A
$$

Proof. We have, on $\left\{|\tau|=b_{i-1}|\lambda|^{\alpha_{i-1}-\infty}\right\}$

$$
\left|\lambda^{-(1-\alpha)\left(l-s_{k}\right)-\beta_{k}} \tau^{l}\right| \leqq C|\lambda|-(1-\alpha)\left(l-s_{k}\right)-\beta_{k}-l\left(\alpha-\alpha_{i-1}\right) .
$$

Since $\quad(1-\alpha)\left(l-s_{k}\right)+\beta_{k}+l\left(\alpha-\alpha_{i-1}\right)$

$$
=\left(1-\alpha_{i-1}\right)\left(l-s_{k}\right)+\left(\alpha-\alpha_{i-1}\right) s_{k}+\beta_{k} \geqq \beta_{k_{i-1}}+\left(\alpha-\alpha_{i-1}\right) s_{k_{i-1}},
$$

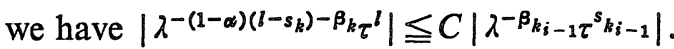

On the other hand we have

$$
\left(1-\alpha_{i}\right)\left(l-s_{k}\right)+\left(\alpha-\alpha_{i}\right) s_{k}+\beta_{k} \geqq\left(\alpha-\alpha_{i}\right) s_{k}+\beta_{k} \geqq \beta_{k_{i-1}}+\left(\alpha-\alpha_{i}\right) s_{k_{i-1}} .
$$

Hence we have on $\left\{|\tau|=b_{i}|\lambda|^{\alpha_{i}{ }^{-\alpha}}\right\} \cup\left\{\lambda^{\alpha_{i}{ }^{-\alpha}} \partial K_{i}(\delta)\right\}$

$$
\left|\lambda^{-(1-\alpha)\left(l-s_{k}\right)-\beta_{k} \tau^{l}}\right| \leqq C\left|\lambda^{-\beta_{k_{i-1}} \tau^{s} k_{i-1}}\right| \text {. }
$$

It follows from Proposition 6.6 that on the boundary of $\tau(i)$

$$
\mid \lambda^{-(1-\alpha)\left(l-s_{k}\right)-\beta_{k} \tau^{l} / G_{0}(z, \lambda, \tau) \mid \leqq A}
$$

By the maximal principle of holomorphic functions implies (6.13) holds on $\tau(i)$.

(III) Sectors $S_{i}(1 \leqq i \leqq p-1)$. In (III) we define sectors $\left\{S_{i} ; 1 \leqq i \leqq p-1\right)$ whose vertex is the origin in $\tau$-space. We make use of the sectors to prove Theorem 1.3 in $\S 8$. To define $S_{i}$ we give two lemmas.

Lemma 6.8. There is an $\omega_{0}\left(\left|\omega_{0}\right|=1\right)$ such that $\arg \left(i_{i, j}(0) \omega_{0}\right) \neq \pi-\pi \alpha_{i}$ $(\bmod 2 \pi)$ for all $1 \leqq i \leqq p-1$ and $1 \leqq j \leqq s_{k_{i-1}}-s_{k_{i}}$.

Proof. Set $B=\left\{\stackrel{\circ}{i}, j_{i}(0) ; 1 \leqq i \leqq p-1,1 \leqq j \leqq s_{k_{j-1}}-s_{k_{i}}\right\}, L_{i}=\left\{r e^{i\left(\pi-\pi \alpha_{i}\right)} ;\right.$ $r \geqq 0\}$ and $L=\cup_{i=1}^{p-1} L_{i} . \quad B$ is a finite set of nonzero points and $L$ is a finite set of half lines. So we can find an $\omega_{0}\left(\left|\omega_{0}\right|=1\right)$ such that $\omega_{0} B \cap L=\phi$. This implies the assertion.

It follows from Lemma 6.8 that

Lemma 6.9. There are $\omega_{1}\left(\left|\omega_{1}\right|=1\right)$ and positive numbers $r$ and $\varepsilon_{1}$ such that 
$\arg \tau_{i, j}(z, \lambda) \omega \neq \pi-\pi \alpha_{i}(\bmod 2 \pi)$ for all $|\lambda| \geqq \Lambda_{0},|z| \leqq r$ and $\left|\omega-\omega_{1}\right|<\varepsilon_{1}$.

Thus we conclude:

Proposition 6.10. There are $\delta, \varepsilon_{1}>0, \hat{z}_{1} \neq 0$ and for each $i(1 \leqq i \leqq p-1)$ an open sector $S_{i}$ with the vertex 0 in $\boldsymbol{C}^{1}$ such that

$$
S_{i} \ni e_{i}=e^{i\left(\pi-\pi \alpha_{i}\right)} \quad \text { and } \quad \bar{S}_{i} \cap\left(-z_{1} K(\delta)\right)=\phi \quad \text { for } \quad\left|z_{1}-\hat{z}_{1}\right|<\varepsilon_{1} .
$$

(IV) Representation of functions in $\mathcal{O}\left(\widetilde{\Omega}\left(\theta_{0}\right)\right.$ ) (Proofs of Lemmas 2.1 and 2.2). Let $w(z) \in \mathcal{O}\left(\tilde{\Omega}\left(\theta_{0}\right)\right)\left(\theta_{0}>\pi\right), \Omega=\{|z| \leqq R\}$. Define for $-\theta_{0}<\theta<\theta_{0}-2 \pi$

$$
\hat{w}_{\theta}\left(\lambda, z^{\prime}\right)=\frac{1}{2 \pi i} \int_{T(\theta)} \exp \left(-\lambda t_{0}\right) w\left(t_{0}, z^{\prime}\right) d t_{0} .
$$

Then $\hat{w}_{\theta}\left(\lambda, z^{\prime}\right)$ is an entire function of $\lambda$ and

$$
\left|\hat{w}_{\theta}\left(\lambda, z^{\prime}\right)\right| \leqq C_{\varepsilon} \exp (\varepsilon|\lambda|) \quad \text { for } \quad|\arg \lambda+\theta|<\pi / 2 .
$$

Lemma 6.11. If $\sup _{\left|t^{\prime}\right| \leqq R}\left|w\left(t_{0}, t^{\prime}\right)\right| \leqq A \exp \left(\delta\left|t_{0}\right|^{-\gamma}\right)(r>0)$, then

$$
\begin{aligned}
& \sup _{||^{\prime} \leqq R}\left|\hat{w}_{\theta}\left(\lambda, t^{\prime}\right)\right| \leqq B \exp \left(2\left(\delta|\lambda|^{\gamma}\right)^{1 /(1+\gamma)}\right) \text { for } \lambda \text { with }|\lambda| \geqq 1 \\
& \text { and }|\arg \lambda+\theta|<\pi / 2 \text {. }
\end{aligned}
$$

Proof. Choose $\varepsilon=\left(\delta|\lambda|^{-1}\right)^{1 /(1+\gamma)}$ in the path $T(\theta)$. Then on $\left|t_{0}\right|=\varepsilon$, $\left|t_{0} \lambda\right|+\delta\left|t_{0}\right|^{-\gamma}=\varepsilon|\lambda|+\delta \varepsilon^{-\gamma}=2\left(\left.\delta|\lambda|\right|^{\gamma}\right)^{1 /(1+\gamma)}$. So we have (6.16).

Set for $|\psi+\theta|<\pi / 2$

$$
w_{\theta}(z)=\frac{1}{2 \pi i} \int_{\Lambda(\psi)} \exp \left(\lambda z_{0}\right) \hat{w}_{\theta}\left(\lambda, z^{\prime}\right)(\log \lambda) d \lambda .
$$

Then $w_{\theta}(z) \in \mathcal{O}(\widetilde{\Omega}(\theta, \theta+2 \pi))$ by (6.15). We have the relation between $w(z)$ and $w_{\theta}(z)$.

Lemma 6.12. $w(z)-w_{\theta}(z)$ is holomorphic in $\left\{z ;\left|z_{0}\right|<R,\left|z^{\prime}\right| \leqq R\right\}$.

Proof. From (6.17) for $z_{0}$ with $\left|\arg z_{0}+\psi-\pi\right|<\pi / 2$

$$
\begin{aligned}
w_{\theta}(z) & =\frac{1}{2 \pi i} \int_{\Lambda(\psi)} \exp \left(\lambda z_{0}\right)(\log \lambda) d \lambda \frac{1}{2 \pi i} \int_{T(\theta)} \exp \left(-\lambda t_{0}\right) w\left(t_{0}, z^{\prime}\right) d t_{0} \\
& =\frac{1}{2 \pi i} \int_{0}^{\infty e^{i \psi}} \exp \left(\lambda z_{0}\right) d \lambda \int_{T(\theta)} \exp \left(-\lambda t_{0}\right) w\left(t_{0}, z^{\prime}\right) d t_{0} .
\end{aligned}
$$

Set $\psi=-\theta$ and let $\arg z_{0}=\theta+\pi$ and $\left|z_{0}\right|>2 \varepsilon$. Then by the definition of $T(\theta)$, we have 


$$
w_{\theta}(z)=\frac{1}{2 \pi i} \int_{T(\theta)}\left\{w\left(t_{0}, z^{\prime}\right) /\left(t_{0}-z_{0}\right)\right\} d t_{0}=w(z)-v(z),
$$

where $v(z)=-\frac{1}{2 \pi i} \int_{T^{*}(\theta)}\left\{w\left(t_{0}, z^{\prime}\right) /\left(t_{0}-z_{0}\right)\right\} d t_{0}$ and $T^{*}(\theta)$ is a path starting at $R e^{i \theta}$ and going around on the circle $|t|=R$ once. So $v(z)$ is holomorphic in $\left\{z ;\left|z_{0}\right|<R,\left|z^{\prime}\right| \leqq R\right\}$. This completes the proof.

We have Lemma 2.1 from Lemma 6.11 and Lemma 2.2 from Lemma 6.12.

\section{§7. Holomorphic Extension of the Kernel Function $w\left(z, \mathbb{t}^{\prime \prime}, \lambda, \zeta\right)$}

We try to analyze the integral representation in detail. To do so we study the singularties of the solution $w\left(z, t^{\prime \prime}, \lambda, \zeta\right)=w^{h}\left(z, t^{\prime \prime}, \lambda, \zeta\right)$ of (3.1). We will obtain more precise informations of the integral representation from them, which yield the results of removable singularities (Theorem 1.3).

Now we always assume

$$
\left\{\begin{array}{l}
\left.\prod_{i=0}^{p-1} A_{k_{i}, s_{k_{i}}}\left(z, \xi^{\prime}\right)\right|_{\xi^{\prime}=\hat{\xi}^{\prime}=(1,0, \cdots, 0)} \neq 0 \text { for }|z| \leqq R_{0}, \\
\sigma_{1}>1 \text { and } s_{k_{p-1}}=0
\end{array}\right.
$$

through $\S 7$ and $\S 8$. This means $p>1$ and (6.3)- $i$ hold for all $0 \leqq i \leqq p-1$. Let us recall the definitions of the path $r(c)$ in $\tau$-space (see $\S 5$-II) and positive constants $a_{i}$ and $b_{i}$ (see Lemma 6.2 and (6.10)). Let us write the sets appearing often in the sequels:

$$
\left\{\begin{aligned}
& \Xi=\left\{\left(z, t^{\prime \prime}, \lambda\right) ;|z| \leqq r, R_{1} \leqq\left|t_{i}\right| \leqq R(i \geqq 2),|\lambda| \geqq \Lambda_{0}\right\} \\
& \tau(i)=\left\{\tau ; b_{i}|\lambda|{ }^{\alpha_{i}-\alpha} \leqq|\tau| \leqq b_{i-1}|\lambda|{ }^{\alpha_{i-1}-\alpha}, \tau \notin \lambda^{\alpha_{i}-\alpha} K_{i}(\delta)\right\} \\
& X(i)=\left\{\left(z, t^{\prime \prime}, \lambda, \tau\right) ;\left(z, t^{\prime \prime}, \lambda\right) \in \Xi, \tau \in \tau(i)\right\}, \\
& Y(i)=\left\{\left(z, t^{\prime \prime}, \lambda, \zeta, \tau\right) ;\left(z, t^{\prime \prime}, \lambda, \tau\right) \in X(i), 0<\left|\zeta+\tau z_{1}\right|<A|\tau|\right\} \\
& Z(i)=\left\{\left(z, t^{\prime \prime}, \lambda, \zeta\right) ;\left(z, t^{\prime \prime}, \lambda\right) \in \Xi\right. \\
&\left.2 a_{i}\left|\lambda^{\alpha_{i}-\alpha} z_{1}\right|<|\zeta|<b_{i-1}|\lambda|{ }^{\alpha_{i-1}-\alpha}\left(A-\left|z_{1}\right|\right)\right\} \\
& Z_{i, i+1}=\left\{\left(z, t^{\prime \prime}, \lambda, \zeta\right) ;\left(z, t^{\prime \prime}, \lambda\right) \in \Xi\right. \\
&\left.|\zeta|<b_{i}|\lambda|^{\alpha_{i}-\alpha}\left(A-\left|z_{1}\right|\right), \zeta \notin-z_{1} \lambda^{\alpha_{i}-\alpha} K_{i}(\delta)\right\}
\end{aligned}\right.
$$

$1 \leqq i \leqq p-1$, for $K_{i}(\delta)$ see $(6.8)-(6.10)$.

By Proposition $5.3 V\left(z, t^{\prime \prime}, \lambda, \zeta, \tau\right)$ is holomorphic in $Y \subset Y(1)$ and we may assume $w\left(z, t^{\prime \prime}, \lambda, \zeta\right)$ is holomorphic in $Z(1)$ (see Proof of Proposition 2.3 in $\S 5$ ). Now that we assume (7.1), we have better results than Proposition 5.3.

Proposition 7. 1. (1). The following estimate holds in $X(1)$ : 


$$
\left|v_{p}\left(z, t^{\prime \prime}, \lambda, \tau\right)\right| \leqq A B^{p+1}|\tau|^{-p-2} p ! .
$$

(2). $V\left(z, t^{\prime \prime}, \lambda, \zeta, \tau\right)$ is holomorphic in $Y(1)$.

If we assume (7.3) in $X(1)$, we can show (2) by the same method as in the proof of Lemma 5.2. The estimate (7.3) is shown $\S 9$. So from Proposition 7.1 we can define, by setting $r_{2}=r\left(b_{1}\right)$,

$$
w_{2}\left(z, t^{\prime \prime}, \lambda, \zeta\right)=\int_{\gamma_{2}} V\left(z, t^{\prime \prime}, \lambda, \zeta, \tau\right) d \tau
$$

By repeating the same argument as in the proof of Proposition 2.3 (see $\S 5-$ II) we have

Proposition 7.2. $w_{2}^{\prime}\left(z, t^{\prime \prime}, \lambda, \zeta\right)$ is holomorphic in $\tilde{Z}_{2}^{\prime}, Z_{2}^{\prime}=\left\{\left(z, t^{\prime \prime}, \lambda, \zeta\right)\right.$; $\left.\left(z, t^{\prime \prime}, \lambda\right) \in \Xi, 2 b_{1}\left|z_{1}\right|<|\zeta|<b_{1}\left(A-\left|z_{1}\right|\right)\right\}$.

Set $w_{1}\left(z, t^{\prime \prime}, \lambda, \zeta\right)=w\left(z, t^{\prime \prime}, \lambda, \zeta\right)$ and

$$
w_{1,2}\left(z, t^{\prime \prime}, \lambda, \zeta\right)=\int_{\gamma_{1}-\gamma_{2}} V\left(z, t^{\prime \prime}, \lambda, \zeta, \tau\right) d \tau
$$

Then we have

$$
w_{1}\left(z, t^{\prime \prime}, \lambda, \zeta\right)=w_{1,2}\left(z, t^{\prime \prime}, \lambda, \zeta\right)+w_{2}\left(z, t^{\prime \prime}, \lambda, \zeta\right) \text { in } \widetilde{Z_{1} \cap Z_{2}^{\prime}} .
$$

Since the path $\left(r_{1}-r_{2}\right)$ can be deformed to the path $\partial K_{1}(\delta)$, we get

Proposition 7.3. $w_{1,2}\left(z, t^{\prime \prime}, \lambda, \zeta\right) \in \mathcal{O}\left(\tilde{Z}_{1,2}\right)$.

We have defined $w_{i}\left(z, t^{\prime \prime}, \lambda, \zeta\right)(i=1,2)$ and $w_{1,2}\left(z, t^{\prime \prime}, \lambda, \zeta\right)$. Let us construct inductively $w_{i}\left(z, t^{\prime \prime}, \lambda, \zeta\right)$ and $w_{i, i+1}\left(z, t^{\prime \prime}, \lambda, \zeta\right)(i=1,2, \cdots, p-1)$ such that

$$
w_{i-1}\left(z, t^{\prime \prime}, \lambda, \zeta\right)=w_{i-1, i}\left(z, t^{\prime \prime}, \lambda, \zeta\right)+w_{i}\left(z, t^{\prime \prime}, \lambda, \zeta\right) .
$$

By the relation (7.7) we shall get the holomorphic extension of $w\left(z, t^{\prime \prime}, \lambda, \zeta\right)$ as a function of $\zeta$ onto a covering space of

$$
Z_{0}=\left\{\zeta ;|\zeta|<b_{0}|\lambda|^{1-\alpha}\left(A-\left|z_{1}\right|\right), \zeta \notin-z_{1} \cup_{q=1}^{p-1} \lambda^{\alpha_{\eta}-\alpha} K_{q}(\delta)\right\} .
$$

Define $r_{i}=r\left(c_{i}\right), 2 a_{i}|\lambda|^{\alpha_{i}-\alpha} \leqq\left|c_{i}\right| \leqq b_{i-1}|\lambda|^{\alpha_{i-1}-\alpha}$. Assume that $w_{i-1}\left(z, t^{\prime \prime}\right.$, $\lambda, \zeta)$ is defined and has the form

$$
w_{i-1}\left(z, t^{\prime \prime}, \lambda, \zeta\right)=\int_{\gamma_{i-1}} V^{i-1}\left(z, t^{\prime \prime}, \lambda, \zeta, \tau\right) d \tau,
$$

where $V^{i-1}\left(z, t^{\prime \prime}, \lambda, \zeta, \tau\right)=\sum_{p=h-1}^{+\infty} v_{p}^{i-1}\left(z, t^{\prime \prime}, \lambda, \tau\right) f_{p}\left(\zeta+\tau z_{1}\right)$ converging in $Y(i-1)$ and $V^{1}\left(z, t^{\prime \prime}, \lambda, \zeta, \tau\right)=V\left(z, t^{\prime \prime}, \lambda, \zeta, \tau\right)$. Let us define $V^{i}\left(z, t^{\prime \prime}, \lambda, \zeta, \tau\right)$. 
Set $v_{p}^{i}\left(z, t^{\prime \prime}, \lambda, \tau\right)=0$ for $p \leqq h-2$ and suppose that $v_{p-j}^{i}\left(z, t^{\prime \prime}, \lambda, \tau\right)(j \geqq 1)$ are defined so that

$$
v_{p-j}^{i}\left(z, t^{\prime \prime}, \lambda, \tau\right)-v_{p-j}^{i-1}\left(z, t^{\prime \prime}, \lambda, \tau\right) \in \mathcal{O}\left(\left\{|\tau| \leqq b_{i-1}|\lambda|^{\alpha_{i-1}{ }^{\alpha}}\right\}\right) .
$$

Set $g_{p}^{i}\left(z, t^{\prime \prime}, \lambda, \tau\right)=\sum_{j=1}^{m} G_{j}\left(z, \lambda, \tau, \partial_{z}\right) v_{p-j}^{i}\left(z, t^{\prime \prime}, \lambda, \tau\right)$. Then $g_{p}^{i}\left(z, t^{\prime \prime}, \lambda, \tau\right)-$ $g_{p}^{i-1}\left(z, t^{\prime \prime}, \lambda, \tau\right) \in \mathcal{O}\left(\left\{|\tau| \leqq b_{i-1}|\lambda|{ }^{\alpha_{i-1}-\alpha}\right\}\right)$. We have

Proposition 7.4. There exists uniquely a polynomial $h_{p}^{i}\left(z, t^{\prime \prime}, \lambda, \tau\right)$ with degree $\leqq s_{k_{i-1}}-1$ such that

$$
\begin{gathered}
\int_{\gamma_{i}} \frac{\left\{g_{p}^{i-1}\left(z, t^{\prime \prime}, \lambda, \tau\right)-g_{p}^{i}\left(z, t^{\prime \prime}, \lambda, \tau\right)+h_{p}^{i-1}\left(z, t^{\prime \prime}, \lambda, \tau\right)\right\} \tau^{l}}{G_{0}(z, \lambda, \tau)} d \tau \\
=\int_{\gamma_{i}} \frac{h_{p}^{i}\left(z, t^{\prime \prime}, \lambda, \tau\right) \tau^{l}}{G_{0}(z, \lambda, \tau)} d \tau \quad \text { for } \quad 0 \leqq l \leqq s_{k_{i-1}}-1 .
\end{gathered}
$$

Proof. There exist $s_{k_{i-1}}$ roots of $G_{0}(z, \lambda, \tau)=0$ in the inside of $r_{i}$ if $|\lambda| \geqq$ $\Lambda_{0}$. Proposition 7.4 follows from Lemma 9.8 in $\S 9$.

So set

$$
v_{p}^{i}\left(z, t^{\prime \prime}, \lambda, \tau\right)=\left\{g_{p}^{i}\left(z, t^{\prime \prime}, \lambda, \tau\right)+h_{p}^{i}\left(z, t^{\prime \prime}, \lambda, \tau\right)\right\} / G_{0}(z, \lambda, \tau) .
$$

Since $g_{p}^{i}\left(z, t^{\prime \prime}, \lambda, \tau\right)-g_{p}^{i-1}\left(z, t^{\prime \prime}, \lambda, \tau\right)+h_{p}^{i}\left(z, t^{\prime \prime}, \lambda, \tau\right)-h_{p}^{i-1}\left(z, t^{\prime \prime}, \lambda, \tau\right) \in \mathcal{O}(\{|\tau|$ $\left.\left.\leqq b_{i-1}|\lambda|^{\alpha_{i-1}-\alpha}\right\}\right)$, it follows from Lemma 9.7 in $\S 9$ that (7.9) means that $v_{p}^{i}\left(z, t^{\prime \prime}, \lambda, \tau\right)-v_{p}^{i-1}\left(z, t^{\prime \prime}, \lambda, \tau\right) \in \mathcal{O}\left(\left\{|\tau| \leqq\left. b_{i-1}|\lambda|\right|^{\alpha_{i-1}-\alpha}\right\}\right)$. For the estimate of $v_{p}^{i}\left(z, t^{\prime \prime}, \lambda, \tau\right)$ we have

\section{Lemma 7.5. It holds that}

$$
\left|v_{p}^{i}\left(z, t^{\prime \prime}, \lambda, \tau\right)\right| \leqq A B^{p+1}|\tau|^{-p-2} p ! \quad \text { in } \quad X(i) .
$$

We refer the proof of Lemma 7.5 to $\S 9$. By Lemma 7.5 we can show the convergence of

$$
V^{i}\left(z, t^{\prime \prime}, \lambda, \zeta, \tau\right)=\sum_{p=h-1}^{+\infty} v_{p}^{i}\left(z, t^{\prime \prime}, \lambda, \tau\right) f_{p}\left(\zeta+\tau z_{1}\right)
$$

and get

Proposition 7.6. $V^{i}\left(z, t^{\prime \prime}, \lambda, \zeta, \tau\right)$ is holomorphic in $Y(i)$.

We define

$$
w_{i}\left(z, t^{\prime \prime}, \lambda, \zeta\right)=\frac{1}{2 \pi i} \int_{\gamma_{i}} V^{i}\left(z, t^{\prime \prime}, \lambda, \zeta, \tau\right) d \tau,
$$

and 


$$
w_{i-1, i}\left(z, t^{\prime \prime}, \lambda, \zeta\right)=\frac{1}{2 \pi i} \int_{\gamma_{i-1}-\gamma_{i}} V^{i-1}\left(z, t^{\prime \prime}, \lambda, \zeta, \tau\right) d \tau
$$

If $|\zeta|>\left|\tau z_{1}\right|$, since $v_{p}^{i}\left(z, t^{\prime \prime}, \lambda, \tau\right)-v_{p}^{i-1}\left(z, t^{\prime \prime}, \lambda, \tau\right) \in \mathcal{O}\left(\left\{|\tau| \leqq b_{i-1}|\lambda|^{\alpha_{i-1}-\alpha}\right\}\right)$, $V^{i-1}\left(z, t^{\prime \prime}, \lambda, \zeta, \tau\right)-V^{i}\left(z, t^{\prime \prime}, \lambda, \zeta, \tau\right) \in \mathcal{O}\left(\left\{\tau ;|\tau| \leqq b_{i-1}|\lambda|{ }^{\alpha_{i-1}-\alpha}\right\}\right)$, So we can replace the integrand in (7.12) by $V^{i-1}\left(z, t^{\prime \prime}, \lambda, \zeta, \tau\right)$. Then we have in $\left(z, t^{\prime \prime}, \lambda, \zeta\right) \in Z(i-1) \cap Z(i)$,

$$
w_{i}\left(z, t^{\prime \prime}, \lambda, \zeta\right)=\frac{1}{2 \pi i} \int_{\gamma_{i}} V^{i-1}\left(z, t^{\prime \prime}, \lambda, \zeta, \tau\right) d \tau
$$

Thus

$$
\begin{gathered}
w_{i-1}\left(z, t^{\prime \prime}, \lambda, \zeta\right)=w_{i-1, i}\left(z, t^{\prime \prime}, \lambda, \zeta\right)+\frac{1}{2 \pi i} \int_{\gamma_{i}} V^{i-1}\left(z, t^{\prime \prime}, \lambda, \zeta, \tau\right) d \tau \\
=w_{i-1, i}\left(z, t^{\prime \prime}, \lambda, \zeta\right)+w_{\imath}\left(z, t^{\prime \prime}, \lambda, \zeta\right) \quad \text { in } \overparen{Z(i-1) \cap Z(i)} .
\end{gathered}
$$

We have

Proposition 7.7. $w_{i-1, i}\left(z, t^{\prime \prime}, \lambda, \zeta\right) \in \mathcal{O}\left(\tilde{Z}_{i-1, i}\right)$ and $w_{i}\left(z, t^{\prime \prime}, \lambda, \zeta\right) \in \mathcal{O}(Z(i))$.

Thus by using $w_{i-1, i}\left(z, t^{\prime \prime}, \lambda, \zeta\right)$ and $w_{i}\left(z, t^{\prime \prime}, \lambda, \zeta\right)$, we can extend $w\left(z, t^{\prime \prime}, \lambda, \zeta\right)=w_{1}\left(z, t^{\prime \prime}, \lambda, \zeta\right) \in \mathcal{O}(\tilde{Z}(1))$ holomorphically into $Z_{0}$,

$$
\begin{aligned}
& Z_{0}=\left\{\left(z, t^{\prime \prime}, \lambda, \zeta\right) ;\left(z, t^{\prime \prime}, \lambda\right) \in \Xi,|\zeta|<b_{0}|\lambda|^{1-\alpha}\left(A-\left|z_{1}\right|\right), \zeta \notin-z_{1} K\right\}, \\
& K=\cup_{q=1}^{p} \lambda^{\alpha_{q}-\alpha} K_{q}(\delta),
\end{aligned}
$$

in the following way:

$$
\begin{aligned}
& w_{1}\left(z, t^{\prime \prime}, \lambda, \zeta\right)=w_{1,2}\left(z, t^{\prime \prime}, \lambda, \zeta\right)+w_{2}\left(z, t^{\prime \prime}, \lambda, \zeta\right) \text { in } \overparen{Z_{1,2} \cap Z(2)} \\
& =w_{1,2}\left(z, t^{\prime \prime}, \lambda, \zeta\right)+w_{2,3}\left(z, t^{\prime \prime}, \lambda, \zeta\right)+w_{3}\left(z, t^{\prime \prime}, \lambda, \zeta\right) \text { in } \widetilde{Z_{2,3} \cap Z(3)} \\
& =w_{1,2}\left(z, t^{\prime \prime}, \lambda, \zeta\right)+w_{2,3}\left(z, t^{\prime \prime}, \lambda, \zeta\right)+\cdots+w_{p-1, p}\left(z, t^{\prime \prime}, \lambda, \zeta\right) \\
& \text { in } \tilde{Z}_{p-1, p} \text {. }
\end{aligned}
$$

Summing up the above extension, we have

Theorem 7.8. $w\left(z, t^{\prime \prime}, \lambda, \zeta\right)$ has a holomorphic prolongation by (7.16) as a function of $\zeta$ to some covering space $\hat{Z}_{0}$ of $Z_{0}$.

We denote this prolonged function also by $w\left(z, t^{\prime \prime}, \lambda, \zeta\right)$. So in (2.2), the definition of $K_{\theta}^{h}\left(z, \lambda, t^{\prime \prime}\right)$, we can deform $C(\theta)$ homotopically to a path in $\hat{Z}_{0}$. We'll perform it in $\S 8$ to show Theorem 1.3.

\section{§ 8. Removability of Singularities}

In this section we assume (7.1) and complete the proof of Theorem 1.3. 
So Propositions in $\S 6$ are available. Sectors $\left\{S_{i}\right\}(1 \leqq i \leqq p-1)$ appearing in this section are those in Proposition 6.10. Set

$$
\Xi^{\prime}=\left\{\left(z, t^{\prime \prime}, \lambda\right) ;\left(z, t^{\prime \prime}, \lambda\right) \in \Xi,\left|z_{1}-\hat{z}_{1}\right|<\varepsilon_{1}\right\},
$$

where $\varepsilon_{1}>0$ and $\hat{z}_{1} \neq 0$ are also those in Proposition 6.10. We always assume $\left(z, t^{\prime \prime}, \lambda\right) \in \Xi^{\prime}$ in this section. In (I)-(II), by using Propositions 6.5 and 6.10 and Theorem 7.8, we decompose $K_{-\pi}^{h}\left(z, t^{\prime \prime}, \lambda\right)$. In (III) we decompose $u(z)$ with the aid of the decomposition of $K_{-\pi}^{h}\left(z, t^{\prime \prime}, \lambda\right)$. In (IV) we complete the proof of Theorem 1.3, by using Theorems 1.7 and 1.9.

(I) Deformation of path $C(\theta)$. We have constructed $K_{\theta}^{h}\left(z, t^{\prime \prime}, \lambda\right)$ in $\S 5$. In view of Theorem 7.8 we can deform the path $C(\theta)$ in the definition of $K_{\theta}^{h}\left(z, t^{\prime \prime}, \lambda\right)((5.7))$. Firstly let us define some paths in $\zeta$-space. For a path $C=\{\zeta(t) ; 0 \leqq t \leqq 1\}$ and $a \in \mathbb{C}, a C=\{a \zeta(t) ; 0 \leqq t \leqq 1\} . A_{i}$ is a straight line which starts at $d_{i-1} e^{-i \pi \alpha_{i-1} \lambda^{\alpha_{i-1}-\alpha}}$ and ends at $c_{i} e^{-i \pi \alpha_{i} \lambda \alpha_{i}-\alpha}\left(c_{i}>2 a_{i}\left|z_{1}\right|>b_{i}\left|z_{1}\right|>\right.$ $d_{i}>0, i=1,2, \cdots, p-1, d_{0}=d, d$ being in (2.12)). $\quad B_{i}$ is a circle starting at $c_{i} e^{-i \pi \alpha_{i}}$ and enclosing $\zeta=0$ once.

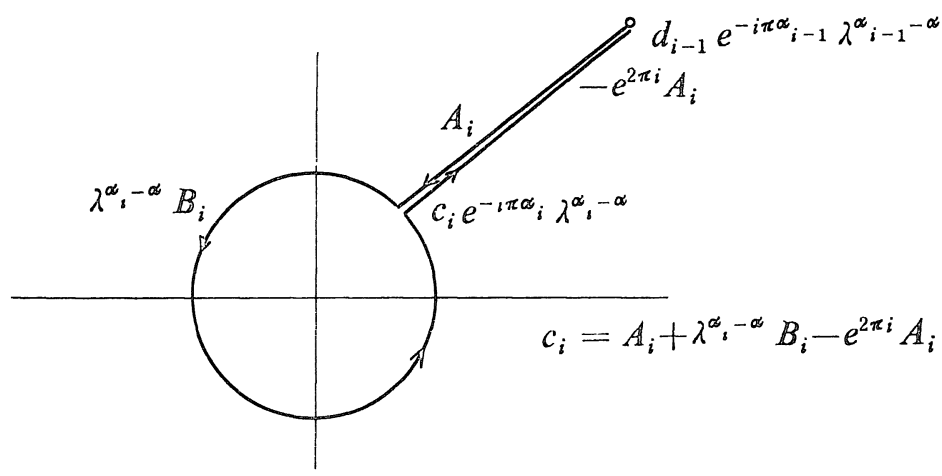

Fig. 8.1.

Set $C_{i}=A_{i}+\lambda^{\omega_{i}-\alpha} B_{i}-e^{2 \pi i} A_{i}$ (see Fig. 8.1.). The singularities of $w^{h}\left(z, t^{\prime \prime}, \lambda, \zeta\right)$ are in the inside of $B_{1}$. So we have

$$
\begin{aligned}
K_{-\pi}^{h}\left(z, t^{\prime \prime}, \lambda\right) & =\int_{C(-\pi)} \exp \left(-\lambda^{\alpha} \zeta\right) w^{h}\left(z, t^{\prime \prime}, \lambda, \zeta\right) d \zeta \\
& =\int_{A_{1}} \cdots d \zeta+\int_{B_{1}} \cdots d \zeta+\int_{-e^{2 \pi i_{1}}} \cdots d \zeta .
\end{aligned}
$$

We note that in general $w^{h}\left(z, t^{\prime \prime}, \lambda, \zeta\right)$ is multi-valued, so in (8.2) $\int_{A_{1}} \ldots d \zeta+$ $\int_{-e^{2 \pi i_{A_{1}}}} \cdots d \zeta \neq 0$. Let us try to deform the path $B_{1}$ to another path. We have 
Proposition 8.1. The path $B_{1}$ in (8.2) can be deformed homotopically to $B_{1}^{\prime}$ containing the path $C_{2}$ as a subpath, $B_{1}^{\prime}=B_{1}^{\prime}(+)+C_{2}+B_{1}^{\prime}(-)$, with the following properties:

The paths $B_{1}^{\prime}(+)$ and $B_{1}^{\prime}(-)$ are in $\left\{d_{1} \leqq|\zeta| \leqq c_{1}\right\},\left(B_{1}^{\prime}(+) \cup B_{1}^{\prime}(-)\right) \cap \overline{S_{1}}=\phi$, $B_{1}^{\prime}(+) \cup B_{1}^{\prime}(-)$ encloses $-z_{1} K_{1}(\delta)$ and

$$
\int_{B_{1}} \exp \left(-\lambda^{\infty} \zeta\right) w^{h}\left(z, t^{\prime \prime}, \lambda, \zeta\right) d \zeta=\int_{B_{1}^{\prime}(+)} \cdots d \zeta+\int_{C_{2}} \cdots d \zeta+\int_{B_{1}^{\prime}(-)} \cdots d \zeta .
$$

Proof. The singularities of $w^{h}\left(z, t^{\prime \prime}, \lambda, \zeta\right)$ lie in $-z_{1} K_{1}(\delta)$ or in $\cup_{q=2}^{p-1}\left\{-z_{1} \lambda^{\alpha_{q}-\alpha} K_{q}(\delta)\right\}$ by Theorem 7.8. The latter singularities are in $\left\{\zeta ;|\zeta|<a_{2}|\lambda|^{\alpha_{2}-\alpha}\left|z_{1}\right|\right\}$, that is, in the inside of $C_{2}$. So we can deform $B_{1}$ so that $B_{1}^{\prime}(+)$ and $B_{1}^{\prime}(-)$ enclose $-z_{1} K_{1}(\delta)$ and from $\left(-z_{1} K_{1}(\delta)\right) \cap \overline{S_{1}}=\phi$ (Proposition 6.10), $\left(B_{1}^{\prime}(+) \cup B_{1}^{\prime}(-)\right) \cap \overline{S_{1}}=\phi$ and (8.3) hold.

The singularities of $w^{h}\left(z, t^{\prime \prime}, \lambda, \zeta\right)$ in the inside of $C_{2}$ are enclosed by $\lambda^{\alpha_{2}-\alpha} B_{2}$, more precisely, in $-z_{1} \lambda^{\alpha_{2}-\alpha} K_{2}(\delta)$ or in $\bigcup_{q=3}^{p-1}\left\{-z_{1} \lambda^{\alpha_{q}-\alpha} K_{q}(\delta)\right\} \subset$ $\left\{\zeta ;|\zeta|<a_{3}|\lambda|^{\alpha_{3}-\alpha}\left|z_{1}\right|\right\}$. We can again deform the path $C_{2}=A_{2}+\lambda^{\alpha_{2}-\alpha} B_{2}+$ ( $\left.-e^{2 \pi i} A_{2}\right)$, not changing $A_{2}$ and $e^{2 \pi i} A_{2}$, to a path $A_{2}+B_{2}^{\prime}+\left(-e^{2 \pi i} A_{2}\right)$, where $B_{2}^{\prime}$ contains $C_{3}$ as a subpath and the similar results to Proposition 8.1 hold (see Fig. 8.2.).

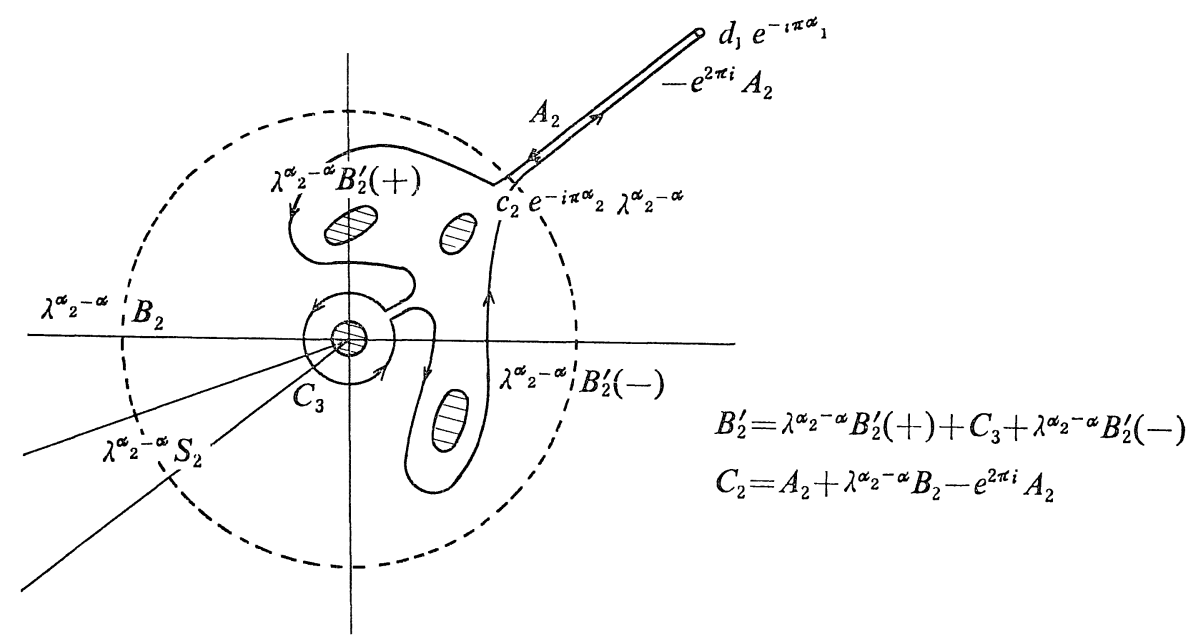

Fig. 8.2.

The singularities of $w^{h}\left(z, t^{\prime \prime}, \lambda, \zeta\right)$ inside of $C_{2}$ are in the parts of oblique lines in Fig. 8.2.

By the repetition of these processes of deformations we have 
Proposition 8.2. The paths $B_{i}(1 \leqq i \leqq p-1)$ can be deformed homotopically in $Z_{0}$ to $B_{i}^{\prime}$ such that

(1) $B_{i}^{\prime}=\lambda^{\alpha_{i}-\alpha} B_{i}^{\prime}(+)+C_{i+1}+\lambda^{\alpha_{i}-\alpha} B_{i}^{\prime}(-)\left(C_{p}=\phi\right)$,

(2) $B_{i}^{\prime}(+)$ and $B_{i}^{\prime}(-)$ are independent of $\lambda$ and contained in $\left\{d_{i} \leqq|\zeta| \leqq c_{i}\right\}$ and $\left\{B_{i}^{\prime}(+) \cup B_{i}^{\prime}(-)\right\} \cap \bar{S}_{i}=\phi$, and it holds that

$$
\begin{aligned}
& \int_{\lambda^{\alpha_{i}-\alpha_{B_{i}}}} \exp \left(-\lambda^{\alpha} \zeta\right) w^{h}\left(z, t^{\prime \prime}, \lambda, \zeta\right) d \zeta=\int_{\lambda^{\alpha_{i}-\alpha_{B_{i}^{\prime}(-)}}} \cdots d \zeta+\int_{C_{i+1}} \cdots d \zeta \\
& \quad+\int_{\lambda^{\alpha_{i}-\alpha_{B_{i}^{\prime}}(-)}} \cdots d \zeta .
\end{aligned}
$$

Thus Proposition 8.2 gives

Proposition 8.3. The kernel function $K_{-\pi}^{h}\left(z, t^{\prime \prime}, \lambda\right)$ is represented in the following form:

$$
\begin{aligned}
& K_{-\pi}^{h}\left(z, t^{\prime \prime}, \lambda\right)=\sum_{i=1}^{p-1}\left\{\int_{A_{i}} \exp \left(-\lambda^{\alpha} \zeta\right) w^{h}\left(z, t^{\prime \prime}, \lambda, \zeta\right) d \zeta\right. \\
& \left.+\int_{-e^{2 \pi i} A_{i}} \cdots d \zeta\right\}+\sum_{i=1}^{p-1}\left\{\int_{\lambda^{\alpha_{i}-\alpha_{B_{i}^{\prime}(+)}}} \cdots d \zeta+\int_{\lambda^{\alpha_{i}-\alpha_{B_{i}^{\prime}(-)}}} \cdots d \zeta\right\} .
\end{aligned}
$$

(II) Decomposition of $K_{-\pi}^{h}\left(z, t^{\prime \prime}, \lambda\right)$. In order to show Theorem 1.3 we further decompose the paths. For this purpose we need lemmas and propositions about the paths $A_{i}, B_{i}^{\prime}(+)$ and $B_{i}^{\prime}(-)$.

Proposition 8.4. Let $\zeta \in A_{i}$ and $\arg \lambda=\pi$. Then there is a $c>0$ such that $\operatorname{Re} \lambda^{\alpha} \zeta \geqq c|\lambda|^{\alpha}{ }^{\alpha}$.

Proof. Since $A_{i}$ is $\zeta(t)=(1-t) d_{i} e^{-i \pi \alpha_{i-1} \lambda^{\alpha_{i-1}-\infty}}+t c_{i} e^{-i \pi \alpha_{i} \lambda^{\alpha_{i}-\alpha}},(0 \leqq t \leqq 1)$, $\operatorname{Re} \lambda^{\alpha} \zeta=(1-t) d_{i}|\lambda|^{\alpha_{i-1}}+t c_{i}|\lambda|^{\alpha_{i}}$. So there is a $c>0$ with $\operatorname{Re} \lambda^{\infty} \zeta \geqq c|\lambda|^{\omega_{i}}$ for $0 \leqq t \leqq 1$.

Lemma 8.5. Let $\zeta=\lambda^{\alpha_{i}-\alpha} \eta, \eta \notin \bar{S}_{i}$. Then there exist $c_{\eta}>0$ and $\psi_{\eta}$ with $\left|\psi_{\eta}-\pi\right|<\pi / 2 \alpha_{i}$ such that $\operatorname{Re} \lambda^{\alpha} \zeta \geqq c_{\eta}|\lambda|{ }^{\alpha}{ }^{i}$ for $\lambda$ with $\arg \lambda=\psi_{\eta}$.

Proof. Set $\lambda=|\lambda| e^{i \psi}$ and $\eta=|\eta| e^{i p}$. Then $\operatorname{Re} \lambda^{\alpha} \zeta=\left|\lambda^{\alpha}{ }^{i} \eta\right| \cos \left(\alpha_{i} \psi+\rho\right)$. Since $\eta \notin S_{i},\left|\rho+\pi \alpha_{i}\right|<\pi-\varepsilon$ for some $\varepsilon>0$. Hence there is a $\psi_{\eta}$ such that $\left|\psi_{\eta}-\pi\right|<\pi / 2 \alpha_{i}$ and $\cos \left(\alpha_{i} \psi_{\eta}+\rho\right)>0$. Thus if $\arg \lambda=\psi_{\eta}, \operatorname{Re} \lambda^{\alpha} \zeta \geqq c_{\eta}|\lambda|{ }^{\alpha_{i}}$ for a $c_{\eta}>0$.

The proof of Lemma 8.5 also shows

Lemma 8.6. Suppose that $K$ is a compact set in $C^{1}$ and $K \cap \overline{S_{i}}=\phi$. If the diameter of $K$ is sufficiently small, then there are $C_{K}>0$ and $\psi_{K}$ with $\left|\psi_{K}-\pi\right|<$ $\pi / 2 \alpha_{i}$ such that $\operatorname{Re} \lambda^{\alpha} \zeta \geqq C_{K}|\lambda|{ }^{\alpha_{i}}$ holds for $\eta \in K$ and $\lambda$ with $\arg \lambda=\psi_{K}$. 
By Lemma 8.6, we can decompose the path $B_{i}^{\prime}( \pm)$.

Proposition 8.7. There are paths $B_{i, s}$ and constants $\psi_{i, s}$ with $\left|\psi_{i, s}-\pi\right|<$ $\pi / 2 \alpha_{i}$ and $c_{i, s}>0\left(1 \leqq s \leqq l_{i}\right)$, which do not depend on $\lambda$ such that

(1) $B_{i}^{\prime}(+)=\sum_{s=1}^{l_{i}^{\prime}} B_{i, s}$ and $B_{i}^{\prime}(-)=\sum_{s=l_{i}^{\prime}+1}^{l_{i}} B_{i, s}$,

(2) $\operatorname{Re} \lambda^{\alpha} \zeta \geqq c_{i, s}|\lambda|{ }^{\alpha_{i}}$ for $\zeta \in \lambda^{\alpha_{i}{ }^{-\alpha}} B_{i, s}$ and $\lambda$ with $\arg \lambda=\psi_{i, s}$.

Let us decompose $K_{-\pi}^{h}\left(z, t^{\prime \prime}, \lambda\right)$ by using the paths $A_{i}$ and $B_{i, s^{*}}$. Set

$$
\begin{aligned}
& K_{-\pi, i, 0}^{h}\left(z, t^{\prime \prime}, \lambda\right)=\left(\int_{A_{i}}+\int_{-e^{2 \pi i} A_{i}}\right) \exp \left(-\lambda^{\alpha} \zeta\right) w^{h}\left(z, t^{\prime \prime}, \lambda, \zeta\right) d \zeta, \\
& K_{-\pi, i, s}^{h}\left(z, t^{\prime \prime}, \lambda\right)=\int_{B_{i, s}(\lambda)} \exp \left(-\lambda^{\alpha} \zeta\right) w^{h}\left(z, t^{\prime \prime}, \lambda, \zeta\right) d \zeta \\
& B_{i, s}(\lambda)=\lambda^{\alpha_{i}-\alpha} B_{i, s} \quad\left(1 \leqq s \leqq l_{i}\right) .
\end{aligned}
$$

Then we have

$$
K_{-\pi}^{h}\left(z, t^{\prime \prime}, \lambda\right)=\sum_{i=1}^{p-1}\left(\sum_{s=0}^{l_{i}} K_{-\pi, i, s}^{h}\left(z, t^{\prime \prime}, \lambda\right)\right) .
$$

It holds for $K_{-\pi, i, s}^{h}\left(z, t^{\prime \prime}, \lambda\right)$ that

Proposition 8.8. The following estimates hold:

$$
\left|K_{-\pi, i, 0}^{h}\left(z, t^{\prime \prime}, \lambda\right)\right| \leqq A \exp \left(B|\lambda|^{\alpha_{i-1}}\right) \quad \text { for } \quad|\arg \lambda-\pi|<\pi / 2,
$$

and

(8.10) $\left|K_{-\pi, i, 0}^{h}\left(z, t^{\prime \prime}, \lambda\right)\right| \leqq A \exp \left(-c|\lambda|^{\alpha_{i}}\right)(c>0) \quad$ for $\lambda$ with $\arg \lambda=\pi$, for $1 \leqq s \leqq l_{i}$

(8.11) $\left|K_{-\pi, i, s}^{h}\left(z, t^{\prime \prime}, \lambda\right)\right| \leqq A \exp \left(B|\lambda|{ }^{\alpha_{i}}\right)$,

(8.12) $\left|K_{-\pi, i, s}^{h}\left(z, t^{\prime \prime}, \lambda\right)\right| \leqq A \exp \left(-\left.c|\lambda|\right|^{\alpha_{i}}\right)(c>0)$ for $\lambda$ with $\arg \lambda=\psi_{i, s}$.

Proof. (8.9) and (8.11) are obvious. We have (8.10) by Proposition 8.4 and (8.12) by Proposition 8.7.

We remark that (8.11) is valid without the condition of the argument of $\lambda$. As we said, $\left(z, t^{\prime \prime}, \lambda\right) \in \Xi^{\prime}$ are assumed. But the estimates (8.9)-(8.11) are also valid for $\left(z, t^{\prime \prime}, \lambda\right) \in \Xi$. The condition $\left(z, t^{\prime \prime}, \lambda\right) \in \Xi^{\prime}$ is required to show (8.12).

(III) Decomposition of $u_{-\pi}^{h}(z)$. By using $K_{-\pi, i, s}^{h}\left(z, t^{\prime \prime}, \lambda\right)$, we divide $u_{-\pi}^{h}(z)$ into the sum of $u_{-\pi, i, s}^{h}(z) . K_{-\pi}^{h}\left(z, t^{\prime \prime}, \lambda\right)$ is single valued by Proposition 2.4 , and $\hat{u}_{-\pi}^{h}\left(\lambda, t^{\prime \prime}\right)$ is an entire function of $\lambda$ by Lemma 2.1. Hence we have from (5.10) 


$$
u_{-\pi}^{h}(z)=\int_{\Lambda_{0} e^{i \pi}}^{\infty} \exp \left(\lambda z_{0}\right) d \lambda \int_{T^{\prime \prime}} K_{-\pi}^{h}\left(z, t^{\prime \prime}, \lambda\right) \hat{u}_{-\pi}^{h}\left(\lambda, t^{\prime \prime}\right) d t^{\prime \prime}+v^{h}(z)
$$

where $v^{h}(z)=\frac{1}{2 \pi i} \int_{|\lambda|=\Lambda_{0}} \exp \left(\lambda z_{0}\right) \log \lambda d \lambda \int_{T^{\prime \prime}} K_{-\pi}^{h}\left(z, t^{\prime \prime}, \lambda\right) \hat{u}_{-\pi}^{h}\left(\lambda, t^{\prime \prime}\right) d t^{\prime \prime}+v_{-\pi}^{h}(z)$, $|\psi-\pi|<\pi / 2$, and $|\lambda|=\Lambda_{0}$ is a path on the circle starting at $-\Lambda_{0}=\Lambda_{0} e^{i \pi}$ and going around once. Set

$$
u_{-\pi, i, s}^{h}(z)=\int_{\Lambda_{0} e^{i \pi}}^{\infty e^{i \psi}} \exp \left(\lambda z_{0}\right) d \lambda \int_{T^{\prime \prime}} K_{-\pi, i, s}^{h}\left(z, t^{\prime \prime}, \lambda\right) \hat{u}_{-\pi}^{h}\left(\lambda, t^{\prime \prime}\right) d t^{\prime \prime} .
$$

Hence we have in $\widetilde{U}(\pi)=\left\{z \in \mathbb{C}^{n+1} ; 0<\left|z_{0}\right| \leqq r,\left|z^{\prime}\right| \leqq r,\left|\arg z_{0}\right|<\pi\right\}$

$$
u(z)=\sum_{(h, i, s)} u_{-\pi, i, s}^{h}(z)+v(z), \quad v(z)=\sum_{h} v^{h}(z) \in \mathcal{O}(U),
$$

and from Proposition 8.8

$\mathbb{P}_{\text {roposition 8.9. }} u_{-\pi, i, s}^{h}(z)\left(1 \leqq s \leqq l_{i}\right)$ are holomorphically extensible to $\widetilde{U}\left(\theta_{0}\right)$ and for any $\theta_{1}$ with $0<\theta_{1}<\theta_{0}$

$$
\left|u_{-\pi, i, s}^{h}(z)\right| \leqq A_{\theta_{1}} \exp \left(c_{\theta_{1}}\left|z_{0}\right|^{-\gamma_{i}}\right) \text { for } z \in \tilde{U}\left(\theta_{1}\right) .
$$

Proof. In the representation of $u_{-\pi, i, s}^{h}(z)((8.14))$ we can deform the path of integration in $\lambda$ by (8.11). Namely we change $\psi$ in (8.14). In doing so we have to replace $\hat{u}_{-\pi}^{h}\left(\lambda, t^{\prime \prime}\right)$ by $\hat{u}_{\theta}^{h}\left(\lambda, t^{\prime \prime}\right)$ and take another holomorphic function $w_{\theta}^{h}(z)$ (see Lemmas 2.2 and 6.12). Consequently if $\theta<\arg z_{0}<\theta+2 \pi\left(-\theta_{0}<\theta<\right.$ $\left.\theta_{0}-2 \pi\right)$, we have

$$
u_{-\pi, i, s}^{h}(z)=\int_{\Lambda_{0} e^{i \pi}}^{\infty} e^{i \psi} \exp \left(\lambda z_{0}\right) d \lambda \int_{T^{\prime \prime}} K_{-\pi, i, s}^{h}\left(z, t^{\prime \prime}, \lambda\right) \hat{u}_{\theta}^{h}\left(\lambda, t^{\prime \prime}\right) d t^{\prime \prime}+w_{\theta}^{h}(z),
$$

$\psi$ being $|\theta-\psi|<\pi / 2$.

Thus we get holomorphic extension of $u_{-\pi, i, s}^{h}(z)$ and (8.16) from (8.11).

Now we use the decay estimate (8.10) and (8.12) and obtain the asymptotic expansion of $u_{-\pi, i, s}^{h}(z)$. In the representation of $u_{-\pi, i, s}^{h}(z)((8.17))$ we choose $\psi=\psi_{i, s}$ in Proposition $8.8\left(\psi_{i, 0}=\pi\right)$. Then we can show that it has the asymptotic expansion with respect to $z_{0}$. Namely,

Proposition 8.10. $u_{-\pi, i, s}^{h}(z)\left(0 \leqq s \leqq l_{i}\right)$ have the asymptotic expansion with bounds with respect to $z_{0}$ in $\left\{z_{0} ;\left|\arg z_{0}+\psi_{i, s}-\pi\right|<\pi / 2\right\}$, that is, there are holomorphic functions $u_{-n, i, s, n}^{h}\left(z^{\prime}\right)(n=0,1, \cdots)$ in $\left\{\left|z^{\prime}\right| \leqq r,\left|z_{1}-\hat{z}_{1}\right|<\varepsilon_{1}\right\}$ and constants $A_{\delta}$ and $B_{\delta}$ such that

$$
\left|u_{-\pi, i, s}^{h}(z)-\sum_{n=0}^{N-1} u_{-\pi, i, s, n}^{h}\left(z^{\prime}\right)\left(z_{0}\right)^{n}\right| \leqq A_{\delta} B_{\delta}^{N}\left|z_{0}\right|^{N} \Gamma\left(N / r_{i}+1\right)
$$


in $\left\{z ; 0<\left|z_{0}\right| \leqq r,\left|\arg z_{0}+\psi_{i, s}-\pi\right|<\pi / 2-\delta,\left|z^{\prime}\right| \leqq r,\left|z_{1}-\hat{z}_{1}\right|<\varepsilon_{1}\right\}$.

Proof. We apply Proposition 10.10 in $\S 10$ to $u_{-\pi, i, s}^{h}(z)$. The inequalities (8.10) and (8.12) imply the condition of Proposition 10.10. Hence we have (8.18).

Since $r=r_{p-1} \leqq r_{i}$, we can say $u_{-\pi, i, s}^{h}\left(t^{-1}, z^{\prime}\right)$ has the $r$-asymptotic expansion with respect to $t$ in $\pi / 2<-\arg t+\psi_{i, s}<3 \pi / 2$.

(IV) Laplace transform of $u(z)$. Now we proceed to complete Theorem 1.3. Here we use the assumption concerning the growth property of $u(z)$. Let us write it again:

For any $\varepsilon>0$ there is a $C_{\varepsilon}>0$ such that

$$
\begin{aligned}
& |u(z)| \leqq C_{\varepsilon} \exp \left(\varepsilon\left|z_{0}\right|^{-\gamma_{p-1}}\right) \text { in } \tilde{\Omega}\left(\theta_{0}\right), \\
& \theta_{0}>\pi\left(1 / 2 \gamma_{p-1}+1\right), r_{p-1}=\sigma_{p-1}-1 .
\end{aligned}
$$

For simplicity we denote $u(z)=u\left(z_{0}, z_{1}, \cdots, z_{n}\right)$ by $u\left(z_{0}\right)$ and $r_{p-1}$ by $r$ because other variables are not important. By setting $z_{0}=t^{-1 / \gamma}$, we have from (8.19)

$$
\left|u\left(t^{-1 / \gamma}\right)\right| \leqq C_{\varepsilon} \exp (\varepsilon|t|) \text { in }\left\{t ;|\arg t|<r \theta_{0},|t|>C^{\gamma}\right\} .
$$

Define the $r$-Laplace transform of $u\left(z_{0}\right), v\left(z_{0}\right)$ and $u_{-\pi, i, s}^{h}\left(z_{0}\right)$ by

$$
\begin{cases}\hat{u}(\xi)=\int_{a}^{+\infty} \exp (\xi t) u\left(t^{-1 / \gamma}\right) t^{-1} d t \quad\left(a>C^{\gamma}\right) . \\ \hat{v}(\xi)=\int_{a}^{+\infty} \exp (\xi t) v\left(t^{-1 / \gamma}\right) t^{-1} d t \quad\left(a>C^{\gamma}\right) . \\ \hat{u}_{-\pi, i, s}^{h}(\xi)=\int_{a}^{\infty} e^{i \varphi} \exp (\xi t) u_{-\pi, i, s}^{h}\left(t^{-1 / \gamma}\right) t^{-1} d t \quad\left(a>C^{\gamma}\right),\end{cases}
$$

where $\pi / 2<-\frac{\varphi}{r}+\psi_{i, s}<3 \pi / 2$ (see Proposition 8.10). We have from (8.21) and Proposition 8.10.

Proposition 8.11.

$$
\text { (a) } \hat{u}(\xi) \in \mathcal{O}\left(\left\{\xi ;|\arg \xi-\pi|<\frac{\pi}{2}+r \theta_{0}\right\}\right) \text {, }
$$

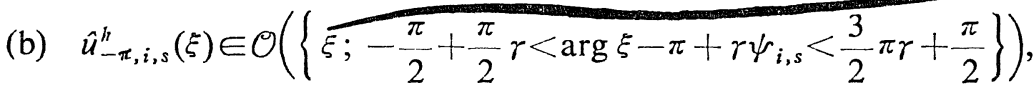

(c) $\hat{v}(\xi) \in \mathcal{O}(\widehat{\{0<|\xi|<\infty\}})$.

By Proposition 8.10 each $u_{-\pi, i, s}^{h}\left(t^{-1}\right)$ has the $r$-asymptotic expansion with respecte to $t$ in $\left\{t \in C^{1} ;|t|>C, \pi / 2<-\arg t+\psi_{i, s}<3 \pi / 2\right\}$ and $v\left(t^{-1}\right)$ is holomorphic at $t=\infty, v\left(t^{-1}\right)=\sum_{n=0}^{\infty} v_{n}\left(z^{\prime}\right) t^{-n}$. This gives informations of the 
behaviours of $\hat{u}_{-\pi, i, s}^{h}(\xi)$ and $\hat{v}(\xi)$ near $\xi=0$, that is, by Theorem 1.7 we have

Proposition 8.12. (a) $\hat{u}_{-\pi, i, s}^{h}(\xi)$ has the holomorphic prolongation onto $\{0<|\xi|<c\}$ for some $c>0$ such that $\hat{u}_{-\pi, i, s}^{h}(\xi) \in \mathcal{O}(\widetilde{0<|\xi|<c\}}),\left|\hat{u}_{-\pi, i, s}^{h}(\xi)\right| \leqq$ $A_{\Theta}|\log \xi|$ in $\{\xi ; 0<|\xi|<c,|\arg \xi|<\Theta\}$ and

$$
\hat{u}_{-\pi, i, s}^{h}(\xi)-\hat{u}_{-\pi, i, s}^{h}\left(\xi e^{2 \pi i}\right)=\sum_{n=0}^{\infty} u_{-\pi, i, s, n}^{h}\left(z^{\prime}\right) \xi^{n / \gamma} / \Gamma(n / \gamma+1),
$$

(b) For $\hat{v}(\xi),|v(\xi)| \leqq A_{\theta, 0}|\log \xi|$ in $\{\xi ; 0<|\xi|<c,|\arg \xi|<\theta\}$

$$
\hat{v}(\xi)-\hat{v}\left(\xi e^{2 \pi i}\right)=\sum_{n=0}^{\infty} v_{n}\left(z^{\prime}\right) \xi^{n / \gamma} / \Gamma(n / \gamma+1) 。
$$

Now let us study the relation between $\hat{u}(\xi), \hat{u}_{-\pi, i, s}^{h}(\xi)$ and $\hat{v}(\xi)$. For this purpose we employ a limitting method (see (8.25)). Firstly we give

Lemma 8.13. There are $\varphi_{i, s}\left(1 \leqq i \leqq p-1,0 \leqq s \leqq l_{i}\right)$ such that

(1) $\varphi_{i, 0}=0$ and $\left|\varphi_{i, s}\right|<\pi r / 2 \gamma_{i}$ for $1 \leqq s \leqq l_{i}$,

(2) $\pi / 2<\left(\varphi_{i, s} / \gamma\right)+\psi_{i, s}<3 \pi / 2$,

where $\psi_{i, 0}=\pi$ and $\psi_{i, s}\left(1 \leqq s \leqq l_{i}\right)$ are those in Proposition 8.7.

Proof. Let us note $\left|\psi_{i, s}-\pi\right|<\pi / 2 \alpha_{i}$. In order that there exist $\varphi_{i, s}$ satisfying (1) and (2), it is necessary and sufficient that $3 \pi / 2-\psi_{i, s}>-\pi / 2 r_{i}$ and $\pi / 2 \gamma_{i}>\pi / 2-\psi_{i, s^{*}}$. This conditions are satisfied by $\psi_{i, s^{*}}$

Choose $\nu_{i}>0(i=1,2, \cdots, p-1)$ such that $\left|\varphi_{i, s}\left(\left(\gamma_{i} / \gamma\right)+\nu_{i}\right)\right|<\pi / 2$ for all $1 \leqq s \leqq l_{i}$. Set

$$
\delta_{0}=\min \left\{\pi / 2+\varphi_{i, s}, \pi / 2-\varphi_{i, s} ; 1 \leqq i \leqq p-1,1 \leqq s \leqq l_{i}\right\}>0 .
$$

Let $\kappa_{i}>0(i=1,2, \cdots, p-1)$. Then we have

$$
\hat{u}(\xi)=\lim _{\kappa_{p-1} \rightarrow 0}\left(\lim _{\kappa_{p-2} \rightarrow 0} \cdots\left(\lim _{\kappa_{1} \rightarrow 0} \int_{a}^{+\infty} \exp \left(\xi t-\sum_{j=1}^{p-1} \kappa_{j} t^{\left(\gamma_{j} / \gamma\right)+\nu_{j}}\right) u\left(t^{-1 / \gamma}\right) t^{-1} d t\right) \cdots\right)
$$

We have from $(8.25)$

Proposition 8.14. Let $|\arg \xi-\pi|<\delta_{0}$. Then

$$
\hat{u}(\xi)=\sum_{h=0}^{s_{m}-1} \sum_{i=1}^{p-1} \sum_{s=0}^{l_{i}} \hat{u}_{-\pi, i, s}^{h}(\xi)+\hat{v}(\xi) .
$$

Proof. Let $s \geqq 1$. Since $\left|u_{-\pi, i, s}^{h}\left(t^{-1 / \gamma}\right)\right| \leqq A_{\theta_{1}} \exp \left(c_{\theta_{1}}|t|^{\gamma_{i} / \gamma}\right)$ in $\{|\arg t|<$ $\left.r \theta_{1}\right\}\left(0<\theta_{1}<\theta_{0}\right)$ by Proposition 8.9, we have

$$
\begin{aligned}
& \bar{u}_{-\pi, i, s}^{h}(\xi) \\
& \quad=\lim _{\kappa_{p-1} \rightarrow 0}\left(\lim _{\kappa_{p-2} \rightarrow 0}\left(\cdots\left(\lim _{\kappa_{i} \rightarrow 0} \int_{a}^{\infty} \exp \left(\xi t-\sum_{j=1}^{p-1} \kappa_{j} t^{\gamma_{j} / \gamma+\nu_{j}}\right) u_{-\pi, i, s}^{h}\left(t^{-1 / \gamma}\right) d t\right) \cdots\right)\right.
\end{aligned}
$$




$$
=\lim _{\kappa_{p-1} \rightarrow 0}\left(\operatorname { l i m } _ { \kappa _ { p - 2 } \rightarrow 0 } \left(\cdots \left(\lim _{\kappa_{i} \rightarrow 0} \int_{a}^{\infty} e^{i \varphi_{i}, s} \exp \left(\xi t-\sum_{j=i}^{p-1} \kappa_{j} t^{\gamma_{j} / \gamma+\nu_{j}}\right) u_{-\pi, i, s}^{h}\left(t^{-1 / \gamma}\right) t^{-1} d t .\right.\right.\right.
$$

Here we use $\left|\varphi_{i, s}\left(\gamma_{i, s} / \gamma+\nu_{i}\right)\right|<\pi / 2$ and $\pi / 2 \leqq-\delta+\varphi_{i, s}<\arg \xi-\pi+\varphi_{i, s}<\delta+\varphi_{i, s}$ $\leqq \pi / 2$. Since $u_{-\pi, i, s}^{h}\left(t^{-1 / \gamma}\right)$ is bounded on $\arg t=\varphi_{i, s}$, we have

$$
\bar{u}_{-\pi, i, s}^{h}(\xi)=\int_{a}^{\infty e^{i \varphi_{i, s}}} \exp (\xi t) u_{-\pi, i, s}^{h}\left(t^{-1 / \gamma}\right) t^{-1} d t=\hat{u}_{\pi, i, s}^{h}(\xi) .
$$

This means (8.26).

Combining Proposition 8.14 with Propositions 8.11 and 8.12 , we have

Proposition 8.15. $\hat{u}(\xi) \in \mathcal{O}\left(\left\{\xi ;|\arg \xi-\pi|<\frac{\pi}{2}+r \theta_{0}\right\}\right)$ and it has the

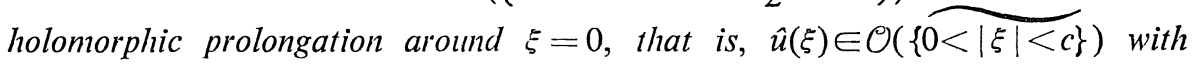
$|\hat{u}(\xi)| \leqq A_{\Theta}|\log \xi|(|\arg \xi|<\Theta)$ and it holds that

$$
\hat{u}(\xi)-\hat{u}\left(\xi e^{2 \pi i}\right)=\sum_{n=0}^{\infty} u_{n}\left(z^{\prime}\right) \xi^{n / \gamma} / \Gamma(n / \gamma+1),
$$

where $u_{n}\left(z^{\prime}\right)=\sum_{(h, i, s)} u_{-\pi, i, s, n}^{h}\left(z^{\prime}\right)+v_{n}\left(z^{\prime}\right)$.

It follows from Proposition 8.15, (8.20) and $\theta_{0}>\pi(1 / 2 \gamma+1)$ that the conditions in Theorem 1.9 are satisfied. So $u\left(t^{-1}\right)=u\left(t^{-1}, z^{\prime}\right)$ is holomorphic at $t=\infty$. This means that $u(z)$ is holomorphic on $\left\{z_{0}=0\right\}$ in $\left\{|z| \leqq r,\left|z_{1}-\hat{z}_{1}\right|<\right.$ $\left.\varepsilon_{1}\right\}$. Hence $\left\{z_{0}=0\right\}$ is removable singularity. Thus we complete the proof of Theorem 1.3.

\section{§ 9. Miscellaneous Results-(II)}

In $\S 9$ we show lemmas and propositions used in the previous sections, but their proofs are not yet given. We give estimates of $v_{p}\left(z, t^{\prime \prime}, \lambda, \tau\right)$ and $h_{p}\left(z, t^{\prime \prime}\right.$, $\lambda, \tau)$, and existence of $h_{p}\left(z, t^{\prime \prime}, \lambda, \tau\right)$ in (I)-(II). For these purposes lemmas about holomorphic functions are given in (II). By proving that $K_{\theta}^{h}(z, \lambda, \tau)$ is single valued, we complete the proof of Proposition 2.4 in (III).

(I) Estimates. We obtain estimates of $v_{p}\left(z, t^{\prime \prime}, \lambda, \tau\right)$ and $h_{p}\left(z, t^{\prime \prime}, \lambda, \tau\right)$ by the method of majorant power series. Let $A(z)=\sum A_{\alpha} z^{\alpha}$ and $B(z)=\sum B_{\alpha} z^{\alpha}$ be formal power series. Then $A(z) \ll B(z)$ means $\left|A_{\alpha}\right| \leqq B_{\alpha}$ for all multiindices $\alpha$. We state elementary properties of majorant power series without the proof, which will be often used. For the proof we refer to [3], [5] and [16].

Lemma 9.1. (Wagschal). Let $\Theta(s)$ be a formal power series of one variable $s$ such that $\Theta(s) \gg 0$ and 


$$
\left(R^{\prime}-s\right) \Theta(s) \gg 0
$$

Then for derivatives, $\Theta^{(j)}(s)=(d / d s)^{j} \Theta(s)(j=0,1, \cdots)$ we have

$$
\left(R^{\prime}-s\right) \Theta^{(j)}(s) \gg 0, \quad \Theta^{(j)}(s) \ll R^{\prime} \Theta^{(j+1)}(s)
$$

and

$$
\left(R_{0}-s\right)^{-1} \Theta^{(j)}(s) \ll\left(R_{0}-R^{\prime}\right)^{-1} \Theta^{(j)}(s)\left(R_{0}>R^{\prime}\right) .
$$

In the following we assume $r<R^{\prime}<R_{0}<R_{1}<R, R_{1} \leqq\left|t_{i}\right| \leqq R$ ( $i \geqq 2$ ), and $|\lambda| \geqq \Lambda_{0}$ and try to obtain estimates of holomorphic functions of $z$, considering $\lambda, \tau, t^{\prime \prime}$ to be parameters. We set $s=z_{0}+z_{1}+\cdots+z_{n}$ and

$$
\theta(s)=\left(R^{\prime}-s\right)^{-1} \text {. }
$$

$\theta(s)$ satisfies the conditions in Lemma 9.1. From Proposition 6.6 we have

Lemma 9.2. Let $\tau \in \tau(i)$. Then

$$
G_{0}(z, \lambda, \tau)^{-1} \ll A|\lambda|^{\beta_{k_{i-1}}}|\tau|^{-s_{k_{i-1}}}\left(R_{0}-s\right)^{-1} .
$$

Let us note $v_{p}\left(z, t^{\prime \prime}, \lambda, \tau\right)=0$ for $p \leqq h-2$ and proceed to obtain estimates of $v_{p}\left(z, t^{\prime \prime}, \lambda, \tau\right)$.

Lemma 9.3. Assume for $\tau \in \tau(1)$ and $j \geqq 1$

$$
v_{p-j}\left(z, t^{\prime \prime}, \lambda, \tau\right) \ll A B^{p-j}|\tau|^{-p+j-2} \theta^{(p-j+1)}(s) .
$$

Then

$$
G_{0}(z, \lambda, \tau)^{-1} g_{p}\left(z, t^{\prime \prime}, \lambda, \tau\right) \ll A C B^{p-1}|\tau|^{-p-2} \theta^{(p+1)}(s) .
$$

Proof. In view of proposition 6.7 and the definition of $G_{j}\left(z, \lambda, \tau, \partial_{z}\right)((3.9))$, we have

$$
\begin{aligned}
& G_{0}(z, \lambda, \tau)^{-1} G_{j}\left(z, \lambda, \tau, \partial_{z}\right) v_{p-j}\left(z, t^{\prime \prime}, \lambda, \tau\right) \\
& \quad \ll A_{1}\left(R_{0}-s\right)^{-1} A B^{p-j}|\tau|^{-p-2} \theta^{(p+1)}(s) \ll A C B^{p-1}|\tau|^{-p-2} \theta^{(p+1)}(s) .
\end{aligned}
$$

From the definition of $g_{p}\left(z, t^{\prime \prime}, \lambda, \tau\right)$ (see (3.10)), we have (9.7).

Lemma 9.4. Under the same assumptions as in Lemma 9.3,

$$
\int_{\gamma} \frac{g_{p}\left(z, t^{\prime \prime}, \lambda, \tau\right)}{G_{0}(z, \lambda, \tau)} \tau^{l} d \tau \ll A C B^{p-1} b_{0}^{-p}|\lambda|^{(\alpha-1)(p+1-l)} \theta^{(p+1)}(s) .
$$

Proof. We can choose the circle $|\tau|=b_{0}|\lambda|^{1-\alpha}$ as the integration path $\gamma$. So (9.8) follows from Lemma 9.3. 
For $C_{p, l}\left(z_{0}, z^{\prime \prime}, t^{\prime \prime}, \lambda\right)$ defined by (4.3),

Lemma 9.5. Under the same assumption as in Lemma 9.3,

$$
C_{p, l}\left(z_{0}, z^{\prime \prime}, t^{\prime \prime}, \lambda\right) \ll A C B^{p-1} b_{0}^{-p}|\lambda|^{(\alpha-1)(p+1-l)} \theta^{(p+1)}(s) .
$$

Proof. We have

$$
v_{p-i}^{(i)}\left(z_{0}, 0, z^{\prime \prime}, t^{\prime \prime}, \lambda, \tau\right)|\tau|^{l-i} \ll A C B^{p-1}|\tau|^{-p-2+l} \theta^{(p+1)}(s) .
$$

Hence, we get in the same way as in Lemma 9.4

$$
\int_{\gamma} v_{p-i}^{(i)}\left(z_{0}, 0, z^{\prime \prime}, t^{\prime \prime}, \lambda, \tau\right)|\tau|^{l-i} d \tau \ll A C B^{p-1}|\lambda|^{(\alpha-1)(p+1-l)} b_{0}^{-p} \theta^{(p+1)}(s) .
$$

Therefore (9.9) follows from the above estimate and Lemma 9.4.

For $h_{p}\left(z, t^{\prime \prime}, \lambda, \tau\right)$ determined by $v_{p-i}\left(z, t^{\prime \prime}, \lambda, \tau\right)(i \geqq 1)$ we have

Lemma 9.6. Let $\tau \in \tau(1)$. Then

$$
h_{p}\left(z, t^{\prime \prime}, \lambda, \tau\right) \ll A C B^{p-1}|\tau|^{-p-2}|\lambda|^{-\kappa(1)} \theta^{(p+1)}(s),
$$

where $x(1)=(\alpha-1) s_{m}$.

The existence of $h_{p}\left(z, t^{\prime \prime}, \lambda, \tau\right)$ and the proof of Lemma 9.6 will be given in (II) by using Lemma 9.5.

We can show Proposition 7.1.

Proof of Proposition 7.1. It follows from Lemma 9.3 and Lemma 9.6 that

$$
\begin{aligned}
& v_{p}\left(z, t^{\prime \prime}, \lambda, \tau\right)=\left\{g_{p}\left(z, t^{\prime \prime}, \lambda, \tau\right)+h_{p}\left(z, t^{\prime \prime}, \lambda, \tau\right)\right\} / G_{0}(z, \lambda, \tau) \\
& \ll A C B^{p-1}|\tau|^{-p-2} \theta^{(p+1)}(s) \ll A B^{p}|\tau|^{-p-2} \theta^{(p+1)}(s) .
\end{aligned}
$$

Thus there is an $r$ such that for $|z| \leqq r$

$$
\left|v_{p}\left(z, t^{\prime \prime}, \lambda, \tau\right)\right| \leqq A B^{p}|\tau|^{-p-2}(p+1) ! .
$$

(II) Lemmas on holomorphic functions. We give some lemmas concerning holomorphic functions and show Lemma 9.6. In (II) we always assume that $f(\tau)$ is a holomorphic functions of one variable $\tau$ in $\left\{\tau \in C^{1} ;|\tau| \leqq R\right\}$, $f(\tau) \neq 0$ on $|\tau|=R$ and the number of zeros of $f(\tau)$ in $\{|\tau|<R\}$ is $s$, the multiplicity being counted.

Lemma 9.7. Let $g(\tau)$ be holomorphic on $\{|\tau| \leqq R\}$ such that

$$
\int_{|\tau|=R} \frac{g(\tau)}{f(\tau)} \tau^{l} d \tau=0 \quad \text { for } \quad 0 \leqq l \leqq s-1 .
$$


Then $g(\tau) / f(\tau)$ is holomorphic on $\{|\tau| \leqq R\}$. In particular if $g(\tau)$ is a polynomial with degree $\leqq s-1$, then $g(\tau) \equiv 0$.

Proof. Let $\tau_{i}(1 \leqq i \leqq q)$ be the distinct zeros of $f(\tau)$ with the multiplicity $\delta_{i}, \sum_{i=1}^{q} \delta_{i}=s$. Hence we have

$$
\frac{g(\tau)}{f(\tau)}=g_{1}(\tau)+\sum_{i=1}^{q}\left\{\sum_{p=1}^{\delta_{i}} \frac{A_{i, p}}{\left(\tau-\tau_{i}\right)^{p}}\right\}
$$

where $g_{1}(\tau)$ is holomorphic on $\{|\tau| \leqq R\}$. Put

$$
u(t)=\int_{|\tau|=R} \exp (t \tau) \frac{g(\tau)}{f(\tau)} d \tau=\sum_{i=1}^{q} \sum_{p=1}^{\delta_{i}} \frac{A_{i, p}}{(p-1) !} t^{p-1} \exp \left(t \tau_{i}\right)
$$

and $f_{1}(\tau)=\prod_{i=1}^{p}\left(\tau-\tau_{i}\right)^{\delta_{i}}$. Then we have $f_{1}(d / d t) u(t)=0$ and from the assumption $(d / d t)^{l} u(0)=0$ for $0 \leqq l \leqq s-1$. Thus it follows from the uniqueness of the Cauchy problem of ordinary differential equations that $u(t)=0$, that is, $A_{i, p}=0$ for all $i$ and $p$. So $g(\tau) / f(\tau)=g_{1}(\tau)$ is holomorphic on $\{|\tau| \leqq R\}$. Now assume $g(\tau)=g_{1}(\tau) f(\tau)$ is a polynomial with degree $\leqq s-1 . g(\tau)$ has $s$ zeros. Hence $g(\tau) \equiv 0$ 。

Lemma 9.8. For any complex numbers $c_{l}(0 \leqq l \leqq s-1)$, there exists uniquely a polynomial $h(\tau)$ with degree $\leqq s-1$ such that

$$
\frac{1}{2 \pi i} \int_{|\tau|=R} \frac{h(\tau)}{f(\tau)} \tau^{l} d \tau=c_{l} \quad \text { for } \quad 0 \leqq l \leqq s-1
$$

Proof. Put $c=\left(c_{0}, c_{1}, \cdots, c_{s-1}\right) \in \mathbb{C}^{s}$. Then the linear mapping defined by (9.15), that is, from the space of all polynomials with degree $\leqq s-1$ to $C^{s}$, is injective by Lemma 9.7. Since the dimensions of these linear spaces are equal, this linear mapping is surjective.

Lemma 9.9. Let $g(\tau)$ be a holomorphic function on $\{|\tau| \leqq R\}$. Then there exists uniquely a polynomial $h(\tau)$ with degree $\leqq s-1$ such that $(g(\tau)-h(\tau)) / f(\tau)$ is holomorphic on $\{|\tau| \leqq R\}$.

Proof. Let $h(\tau)$ be a polynomial with degree $\leqq s-1$ such that

$$
\int_{|\tau|=R} \frac{h(\tau)}{f(\tau)} \tau^{l} d \tau=\int_{|\tau|=R} \frac{g(\tau)}{f(\tau)} \tau^{l} d \tau \quad \text { for } \quad 0 \leqq l \leqq s-1
$$

whose existence and uniqueness follow from Lemma 9.8. By Lemma $9.7(h(\tau)$ $-g(\tau)) / f(\tau)$ is holomorphic on $\{|\tau| \leqq R\}$.

We apply Lemmas 9.7-9.9 to holomorphic functions of $\tau$ with holomorphic 
parameters $\left(z, t^{\prime \prime}, \lambda\right)$ and we can easily show the existence of $h_{p}\left(z, t^{\prime \prime}, \lambda, \tau\right)$ in $\S 4$ and $h_{p}^{i}\left(z, t^{\prime \prime}, \lambda, \tau\right)$ in Proposition 7.4.

Now let us proceed to obtain the estimate of $h_{p}\left(z, t^{\prime \prime}, \lambda, \tau\right)$, namely, the proof of Lemma 9.6. Let $G(z, \lambda, \tau)$ be a holomorphic function of $(z, \lambda, \tau)$ in $X=\left\{(z, \lambda, \tau) \in \boldsymbol{C}^{n+1} \times \boldsymbol{C}^{1} \times \boldsymbol{C}^{1} ;|z| \leqq R_{0},|\tau| \leqq b|\lambda|^{\delta},|\lambda| \geqq \Lambda_{0}\right\}$. We assume that there is a $\kappa \neq 0$ such that

$$
G\left(z, \lambda, b \lambda^{\delta} \mu\right)=\lambda^{-\kappa} \hat{G}(z, \lambda, \mu),
$$

and $\lim _{\lambda \rightarrow \infty} \hat{G}(z, \lambda, \mu)=\hat{G}(z, \infty, \mu)$ exists uniformly in $\left\{(z, \mu) ;|z| \leq R_{0},|\mu| \leq 1\right\}$. We also denote by $\hat{G}(z, \lambda, \mu)$ this extension to $\lambda=\infty$. We add assumptions on $\hat{G}(z, \lambda, \mu)$

$$
|\hat{G}(z, \lambda, \mu)| \geqq c>0 \quad \text { on } \quad|\mu|=1,
$$

and $\hat{G}(z, \lambda, \mu)=0$ has exactly $s$ zeros in $\{|\mu|<1\}$ for any $(z, \lambda)$. Consider the equation

$$
\int_{\gamma} \frac{h(z, \lambda, \tau)}{G(z, \lambda, \tau)} \tau^{l} d \tau=C_{l}(z, \lambda) \quad \text { for } \quad 0 \leqq l \leqq s-1,
$$

where $r$ is a circle starting at $b \lambda^{\delta}$ and ending at $b \lambda^{\delta} e^{2 \pi i}$. Assume that $C_{l}(z, \lambda)$ is holomorphic in $\left\{(z, \lambda) ;|z| \leqq R_{0},|\lambda| \geqq \Lambda_{0}\right\}$ and satisfies

$$
C_{l}(z, \lambda) \ll A B(\lambda)|\lambda|^{\delta l} \Theta(s),
$$

where $\Theta(s)$ satisfying the conditions in Lemma 9.1.

Lemma 9.10. There is a unique polynomial $h(z, \lambda, \tau)$ of $\tau$ with degree $\leqq s-1$ satisfying (9.18) such that for $|\tau| \leqq b|\lambda|^{\delta}$

$$
h(z, \lambda, \tau) \ll A_{1} B(\lambda)|\lambda|^{-\kappa-\delta} \Theta(s),
$$

where $A_{1}$ is independent of $\lambda$ and $B(\lambda)$ is that in (9.19).

Proof. Set $h(z, \lambda, \tau)=\sum_{k=0}^{s-1} A_{k}(z, \lambda) \tau^{k}$. Then (9.18) is equivalent to the algebraic equation

$$
\sum_{k=0}^{s-1} A_{k}(z, \lambda) \int_{\gamma} \frac{\tau^{k+l}}{G(z, \lambda, \tau)} d \tau=C_{l}(z, \lambda) \quad \text { for } \quad 0 \leqq l \leqq s-1 .
$$

By putting $\tau=b \lambda^{\delta} \mu$, we have

$$
\sum_{k=0}^{s-1} A_{k}(z, \lambda)\left(b \lambda^{\delta}\right)^{k+1} \int_{|\mu|=1} \frac{\mu^{k+l}}{\hat{G}(z, \lambda, \mu)} d \mu=C_{l}(z, \lambda)\left(b \lambda^{\delta}\right)^{-l} \lambda^{-\kappa} .
$$

It follows from Lemma 9.8 that 


$$
D(z, \lambda)=\operatorname{det} .\left(\int_{|\mu|=1} \frac{\mu^{k+l}}{\hat{G}(z, \lambda, \mu)} d \mu, 0 \leqq k, l \leqq s-1\right) \neq 0
$$

From (9.17) there is a $c_{0}>0$ such that $|D(z, \lambda)| \geqq c_{0}$ in $\left\{(z, \lambda) ;|z| \leqq R_{0},|\lambda| \geqq\right.$ $\left.\Lambda_{0}\right\}$. Thus $D(z, \lambda)^{-1} \ll C\left(R_{0}-s\right)^{-1}$. By the Cramer's formula we have $A_{k}(z, \lambda)$ $\left(b \lambda^{\delta}\right)^{k+1} \ll C B(\lambda)|\lambda|^{-\kappa} \Theta(s)$. Hence, if $|\tau| \leqq b|\lambda|^{\delta}$, we have $h(z, \lambda, \tau)=\sum_{k=0}^{s-1} A_{k}$ $(z, \lambda) \tau^{k} \ll A_{1} B(\lambda)|\lambda|^{-k-\delta} \Theta(s)$.

Thus we can show Lemma 9.6.

Proof of Lemma 9.6. From Lemma 9.5, by putting $\delta=1-\alpha$ and $B(\lambda)=$ $B^{p-1} b_{0}^{-p}|\lambda|^{-\delta(p+1)}$, we have $C_{p, l}\left(z_{0}, z^{\prime \prime}, \lambda, \tau\right) \ll A B(\lambda)|\lambda|^{\delta l} \theta^{(p+1)}(s)$. Hence by Lemma 9.10, by putting $G(z, \lambda, \tau)=G_{0}(z, \lambda, \tau)$, we have $h(z, \lambda, \tau) \ll A_{1} B(\lambda)$ $|\lambda|^{-\kappa(1)-\delta} \theta^{(p+1)}(s)$ for $|\tau| \leqq b_{0}|\lambda|^{\delta}$. Therefore, if $|\tau| \leqq b_{0}|\lambda|^{\delta}$, we have $\tau^{p+2}$ $h(z, \lambda, \tau) \ll A B^{p}|\lambda|^{-\kappa(1)} \theta^{(p+1)}(s)$. This implies (9.10).

Thus we complete the proof of Proposition 7.1. We can also show Proposition 5.1 in the same way. Because the estimates in Propositions 6.6 and 6.7 and Lemma 9.6 are valid for $\tau \in\left\{a_{1} \leqq|\tau| \leqq b_{0}|\lambda|^{1-\alpha}\right\}$ under the condition $A_{m, s_{m}}\left(0, \hat{\xi}^{\prime}\right) \neq 0$.

By the same method we can obtain Lemma 7.5, the estimate of $v_{p}^{i}\left(z, t^{\prime \prime}, \lambda, \tau\right)$ in $X(i)$. In that case, instead of Lemma 9.6, we adopt:

Lemma 9.11. Let $\tau \in \tau(i)$. Then

$$
h_{p}^{i}\left(z, t^{\prime \prime}, \lambda, \tau\right) \ll A C B^{p-1}|\tau|^{-p-2} \lambda^{-\kappa(i)} \theta^{(p+1)}(s),
$$

where $\kappa(i)=\beta_{k_{i-1}}+\left(\alpha-\alpha_{i-1}\right) s_{k_{i-1}}$.

The proof of Lemma 9.11 is similar to that of Lemma 9.6.

(III) Single valued function $K_{\theta}^{h}\left(z, t^{\prime \prime}, \lambda\right)$. In (III) we complete the proof of Proposition 2.4, by showing that $K_{\theta}^{h}\left(z, t^{\prime \prime}, \lambda\right)$ is single valued, which is not yet proved. In the following the upper suffix $*$ of a function of $\lambda$, for example $f^{*}(\lambda, \cdot)$, means it is a single valued holomorphic function of $\lambda$ on $\left\{\lambda ;|\lambda| \geqq \Lambda_{0}\right\}$. Set $v_{p}(\lambda, \rho)=v_{p}\left(z, t^{\prime \prime}, \lambda, \zeta, \rho \lambda^{1-\alpha}\right)$. Then we have

Proposition 9.12. $v_{p}(\lambda, \rho)$ has the form

$$
v_{p}(\lambda, \rho)=\lambda^{\alpha(p+2)} v_{p}^{*}(\lambda, \rho) .
$$

Let us show (9.22) by induction on $p . v_{k}(\lambda, \rho) \equiv 0$ for $k \leqq h-2$. We need lemmas. 
Lemma 9.13. Assume $v_{k}(\lambda, \rho)$ has the form (9.22) for $k \leqq p-1$. Then

$$
\begin{gathered}
\left.G_{j}\left(z, \lambda, \tau ; \partial_{z}\right)\right|_{\tau=\rho \lambda^{1-\alpha}}=\lambda^{\alpha\left(j-s_{m}\right)} G_{j}^{*}\left(z, \lambda, \rho ; \partial_{z}\right), \\
\left.g_{p}(\lambda, \rho) \equiv \sum_{j=1}^{m} G_{j}\left(z, \lambda, \tau ; \partial_{z}\right)\right|_{\tau=\rho \lambda^{1-\alpha}} v_{p-j}(\lambda, \rho)=\lambda^{\alpha\left(p+2-s_{m}\right)} g_{p}^{*}(\lambda, \rho),
\end{gathered}
$$

where the coefficients of $G_{j}^{*}\left(z, \lambda, \rho ; \partial_{z}\right)$ are single valued with respect to $\lambda$.

Proof. (9.23) and (9.24) follow from (3.9) and (3.10)

For $C_{p, l}\left(z_{0}, z^{\prime \prime}, t^{\prime \prime}, \lambda\right)$ defined by (4.3) we have

Lemma 9.14. Under the same assumption as in Lemma 9.13,

$$
C_{p, l}\left(z_{0}, z^{\prime \prime}, t^{\prime \prime}, \lambda\right)=\lambda^{\alpha(p-l+1)} C_{p, l}^{*}\left(z_{0}, z^{\prime \prime}, t^{\prime \prime}, \lambda\right) .
$$

Proof. We have $v_{p-i}^{(i)}(\lambda, \rho) \lambda^{(1-\alpha)(l-i)}=\lambda^{\alpha(p+2-l)} v_{p, i}^{*}(\lambda, \rho)$. Hence

$$
C_{p, l}\left(z_{0}, z^{\prime \prime}, t^{\prime \prime}, \lambda\right)=\lambda^{\alpha(p+1-l)} c_{p, l}^{*}(\lambda)+\delta_{p, l-1} \delta_{l, h}(2 \pi i)^{-n+1} \Pi_{i=2}^{n}\left(t_{i}-z_{i}\right)^{-1} .
$$

If $p \neq l-1$, we have $C_{p, l}\left(z_{0}, z^{\prime \prime}, t^{\prime \prime}, \lambda\right)=\lambda^{\alpha(p+1-l)} c_{p, l}^{*}(\lambda)$. If $p=l-1$, then the assertion is also valid.

Set $h_{p}(\tau)=h_{p}\left(z, t^{\prime \prime}, \lambda, \tau\right)=\sum_{k=0}^{s=1} A_{p, k}(\lambda) \tau^{k}$, where $s=s_{m}$ and $A_{p, k}(\lambda)=A_{p, k}$ $\left(z, t^{\prime \prime}, \lambda\right)$. We have

Lemma 9.15. Under the same assumption as in Lemma 9.13,

$$
\left.h_{p}(\tau)\right|_{\tau=\rho \lambda^{1-\alpha}}=\lambda^{\alpha(p-s+2)} H_{p}^{*}(\lambda) .
$$

Proof. $h_{p}(\tau)$ is determined by the equation

$$
\int_{\gamma} \frac{h_{p}(\tau)}{G_{0}(\tau)} \tau^{l} d \tau=C_{p, l}(\lambda)
$$

Since

$$
\int_{\gamma} \frac{\tau^{k+l}}{G_{0}(\tau)} d \tau=\lambda^{(1-\alpha)(k+l-s+1)} \int_{\gamma(b)} \frac{\rho^{k+l}}{G_{0}^{*}(\lambda, \rho)} d \rho=\lambda^{-\alpha(k+l-s+1)} H_{k, l}^{*}(\lambda),
$$

the equation becomes $\sum_{k=0}^{s-1} A_{p, k}(\lambda) \lambda^{-\alpha(k+l-s+1)} H_{k, l}^{*}(\lambda)=\lambda^{\alpha(p-l+1)} C_{p, l}^{*}(\lambda)$. Put $A_{p, k}(\lambda)=\lambda^{\alpha(p+k-s+2)} A_{p, k}^{\prime}(\lambda)$. Then $\sum_{k=0}^{s-1} A_{p, k}^{\prime}(\lambda) H_{k, l}^{*}(\lambda)=C_{p, l}^{*}(\lambda)$. Hence $A_{p, k}^{\prime}(\lambda)$ is a single valued holomorphic function of $\lambda$. Thus we have (9.26).

Proof of Proposition 9.12. We have (9.22) from Lemmas 9.13 and 9.15 and $v_{p}\left(z, t^{\prime \prime}, \lambda, \tau\right)=\left\{g_{p}\left(z, t^{\prime \prime}, \lambda, \tau\right)+h_{p}\left(z, t^{\prime \prime}, \lambda, \tau\right)\right\} / G_{0}(z, \lambda, \tau)$.

Set

$$
I_{p}(\lambda)=\int_{C(\theta)} \exp \left(-\lambda^{\alpha} \zeta\right) d \zeta \int_{\gamma\left(b \lambda^{1-\alpha}\right)} v_{p}(\lambda, \tau) f_{p}\left(\zeta+\tau z_{1}\right) d \tau
$$


For $C(\theta)=C\left(d e^{i \theta} \lambda^{1-\alpha}\right)$ see $§ 2$-III. Then

Lemma 9.16. $I_{p}(\lambda)$ is a single valued holomorphic function on $\left\{\lambda ;|\lambda| \geqq \Lambda_{0}\right\}$.

Proof. Put $\tau=\rho \lambda^{1-\alpha}$. Then

$$
\begin{aligned}
& I_{p}(\lambda)=\int_{C(\theta)} \exp \left(-\lambda^{\alpha} \zeta\right) d \zeta \int_{\gamma(b)} v_{p}\left(\lambda, \rho \lambda^{1-\alpha}\right) f_{p}\left(\zeta+\rho \lambda^{1-\alpha} z_{1}\right) \lambda^{1-\alpha} d \rho \\
& =\int_{\gamma(b)} v_{p}\left(\lambda, \rho \lambda^{1-\alpha}\right) \lambda^{1-\alpha} d \rho \int_{C(\theta)} \exp \left(-\lambda^{\alpha} \zeta\right) f_{p}\left(\zeta+\rho \lambda^{1-\alpha} z_{1}\right) d \zeta \\
& =\int_{\gamma(b)} v_{p}\left(\lambda, \rho \lambda^{1-\alpha}\right) \lambda^{1-\alpha} d \rho \int_{-\rho \lambda^{1-\alpha} z_{1}}^{d e^{i \theta \lambda^{1-\alpha}}} \exp \left(-\lambda^{\alpha} \zeta\right)\left\{\left(\zeta+\rho \lambda^{1-\alpha} z_{1}\right)^{p} / p !\right\} d \zeta
\end{aligned}
$$

Since

$$
\begin{aligned}
& \int_{-\rho \lambda^{1-\alpha_{z_{1}}}}^{d e^{i \theta} \lambda^{1-\alpha}} \exp \left(-\lambda^{\alpha} \zeta\right)\left\{\left(\zeta+\rho \lambda^{1-\alpha} z_{1}\right)^{p} / p !\right\} d \zeta \\
& \quad=\lambda^{(1-\alpha)(p+1)} \exp \left(\lambda \rho z_{1}\right) \int_{0}^{d e^{i \theta}+\rho_{z_{1}}} \exp (-\lambda \eta) \eta^{p} / p ! d \eta,
\end{aligned}
$$

we have

$$
I_{p}(\lambda)=\int_{\gamma(b)} v_{p}\left(\lambda, \rho \lambda^{1-\alpha}\right) \lambda^{(1-\alpha)(p+2)} \exp \left(\lambda \rho z_{1}\right) d \rho \int_{0}^{d e^{i \theta+\rho z_{2}}} \exp (-\lambda \eta) \eta^{p} / p ! d \eta .
$$

This means that $I_{p}(\lambda)$ is single valued.

From Proposition $9.12 K_{\theta}^{h}\left(z, t^{\prime \prime}, \lambda\right)=\sum_{p=h-1}^{+\infty} I_{p}(\lambda)$ is also single valued with respect to $\lambda$.

\section{$\S 10$. Function with Asymptotic Expansion}

In $\S 10$ we consider functions with asymptotic expansions and give the proofs of Theorems 1.7 and 1.9. Let $u(t)$ be a continuous function on $[A,+\infty)$ $(A>0)$ such that $|u(t)| \leqq C \exp \left(B|t|^{\gamma}\right)(r>0)$. We have defined the $r$-Laplace transform $\hat{u}(\xi)$ of $u(t)$ by (1.12) and its inversion formula by (1.13) in $\S 1$. Let us recall the notation $\tilde{S}(a, b)=\left\{t \in \mathbb{C}^{1} ;|t| \geqq A, a<\arg t<b\right\}(A>0)$ and $\tilde{S}(a)$ $=\tilde{S}(-a, a)(a>0)$.

Now suppose that $u(t)$ has the $r$-asymptotic expansion (1.14),

$$
u(t) \sim \sum_{k=0}^{+\infty} c_{k} t^{-k} \text { on }[A,+\infty) .
$$

We have $\left|c_{N}\right| \leqq A_{1} R^{-N} \Gamma(N / \gamma+1)$ (see Definition 1.6). By using the sequence $\left\{c_{k}\right\}(k=0,1, \cdots)$, define

$$
g(z)=\sum_{k=0}^{+\infty} \frac{c_{k} z^{k}}{\Gamma(k / r+1)}
$$


and

$$
g_{n}(z)=\sum_{k=0}^{n} \frac{c_{k} z^{k}}{\Gamma(k / \gamma+1)}
$$

We have

Lemma 10.1. (i) $g(z)$ is holomorphic in $\{|z|<R\}$ and $|g(z)| \leq A_{1}$ $(1-|z| R \mid)^{-1}$.

(ii) Let $0<r<R$. Then $\left|g_{n}(z)\right| \leqq A_{r}|z / r|^{n+1}$ for $|z| \geqq r$ and $\left|g(z)-g_{n}(z)\right| \leqq A_{r}$ $\left.|z| R\right|^{n+1}$ for $|z| \leqq r$.

Proof. It follows from the estimates of $c_{k}$ that $g(z)$ is holomorphic in $\{|z|<R\}$ and $|g(z)| \leq A_{1}(1-|z| R \mid)^{-1}$. Since $\left|g_{n}(z)\right| \leqq\left. A_{1} \sum_{k=0}^{n}|z| R\right|^{k}$, if $|z| \geqq r,\left|g_{n}(z)\right| \leqq\left. A_{r}|z| r\right|^{n+1}$. For $|z| \leq r,\left|g(z)-g_{n}(z)\right| \leqq A_{1} \sum_{k=n+1}^{\infty}|z|^{k} R^{-k}$ $\leqq\left. A_{r}|z| R\right|^{n+1}$.

Set

$$
v(t)=t^{\gamma} \int_{0}^{c} \exp \left(-t^{\gamma} z\right) g\left(z^{1 / \gamma}\right) d z, \quad 0<c^{1 / \gamma}<R .
$$

Proposition 10.2. (i) $\mathrm{r}(t) \in \mathcal{O}\left(\widetilde{\boldsymbol{C}^{1}-\{0\}}\right)$.

(ii) $v(t)$ has the $\gamma$-asymptotic expansion as $t \rightarrow \infty$ in $\tilde{S}(\pi / 2 \gamma)$, that is, there is an $A(c)$ such that for $t \in \tilde{S}(\pi / 2 \gamma), t=|t| e^{i \varphi}$,

(10.5) $\left|v(t)-\sum_{k=0}^{N-1} c_{k} t^{-k}\right| \leqq A(c) c^{-N / \gamma}(\cos (\gamma \varphi))^{-N / \gamma-1} \Gamma(N / \gamma+1) \mid t^{-N}$

holds for each $N$.

Proof. Obviously $v(t) \in \mathcal{O}\left(\widetilde{\mathbb{C}^{1}-\{0\}}\right)$. Let us show $v(t)$ has the asymptotic expansion (10.5). We have

$$
\begin{aligned}
v(t) & =t^{\gamma} \int_{0}^{+\infty} \exp \left(-t^{\gamma} z\right) g_{N-1}\left(z^{1 / \gamma}\right) d z \\
& +t^{\gamma} \int_{0}^{c} \exp \left(-t^{\gamma} z\right)\left\{g\left(z^{1 / \gamma}\right)-g_{N-1}\left(z^{1 / \gamma}\right)\right\} d z \\
& -t^{\gamma} \int_{c}^{+\infty} \exp \left(-t^{\gamma} z\right) g_{N-1}\left(z^{1 / \gamma}\right) d z .
\end{aligned}
$$

By a simple calculation we have $t^{\gamma} \int_{0}^{+\infty} \exp \left(-t^{\gamma} z\right) g_{N-1}\left(z^{1 / \gamma}\right) d z=\sum_{k=0}^{N-1} c_{k} t^{-k}$. From (ii) in Lemma 10.1, we have

$$
\begin{aligned}
& \left|t^{\gamma} \int_{0}^{c} \exp \left(-t^{\gamma} z\right)\left\{g\left(z^{1 / \gamma}\right)-g_{N-1}\left(z^{1 / \gamma}\right)\right\} d z\right| \\
& \quad \leqq A_{c} R^{-N}(\cos (\gamma \varphi))^{-N / \gamma-1} \Gamma(N / \gamma+1)|t|^{-N}
\end{aligned}
$$


For the third term in the right hand side in (10.6),

$$
\begin{aligned}
& \left|t^{\gamma} \int_{c}^{+\infty} \exp \left(-t^{\gamma} z\right) g_{N-1}\left(z^{1 / \gamma}\right) d z\right| \\
& \quad \leqq A\left|t^{\gamma}\right| \int_{c}^{+\infty}\left|\exp \left(-t^{\gamma} z\right)\right|\left|\frac{z}{c}\right|^{N / \gamma}|d z| \leqq \frac{A}{\cos (\gamma \varphi)} \frac{\Gamma(N / \gamma+1)}{\left(c^{1 / \gamma}|t| \cos (\gamma \varphi)^{1 / \gamma}\right)^{N}} .
\end{aligned}
$$

Thus we have

$$
\left|v(t)-\sum_{k=0}^{N-1} c_{k} t^{-k}\right| \leqq A(c) c^{-N / \gamma}(\cos (\gamma \varphi))^{-N / \gamma-1} \Gamma(N / \gamma+1)|t|^{-N} .
$$

Set $w(t)=u(t)-v(t)$. From the assumption on $u(t)$ and Proposition 10.2 $w(t) \sim 0$ as $t \rightarrow+\infty$ on the positive real axis. More precisely $|w(t)| \leqq A(c)$ $c^{-n / \gamma} \Gamma(n / \gamma+1)|t|^{-n}$ for each $n$. This implies

Lemma 10.3. The estimate $|w(t)| \leqq C\left(c t^{\gamma}\right)^{1 / 2} \exp \left(-c t^{\gamma}\right)$ holds for $A \leqq t<$ $+\infty$, where $C$ depends only on $c$.

Proof. By Stirling's formula, we have

$$
|w(t)| \leqq B(c)\left|c^{1 / \gamma} t\right|^{-n} \exp (-n / \gamma)(n / \gamma)^{(n / \gamma)+1 / 2} \text {. }
$$

So, if $n / r \leqq c t^{\gamma} \leqq(n+1) / \gamma,|w(t)| \leqq C\left(c t^{\gamma}\right)^{1 / 2} \exp \left(-c t^{\gamma}\right)$.

Let us investigate the $r$-Laplace transform $\hat{u}(\xi)$ of $u(t)$ with the asymptotic expansion (10.1). Since $\hat{u}(\xi)=\hat{v}(\xi)+\hat{w}(\xi)$, we study $\hat{v}(\xi)$ and $\hat{w}(\xi)$. By Lemma 10.3 we have

Lemma 10.4. $\hat{w}(\xi) \in \mathcal{O}(\{\xi ; \operatorname{Re} \xi<c\})$.

On the other hand $\hat{v}(\xi)$ is represented as follows:

$$
\hat{v}(\xi)=\int_{a}^{+\infty} \exp (\xi t) d t \int_{0}^{c} \exp (-t z) g\left(z^{1 / \gamma}\right) d z=\int_{0}^{c} \frac{e^{-a(z-\xi)} g\left(z^{1 / \gamma}\right)}{z-\xi} d z
$$

Hence we have

Proposition 10.5. (i) $\hat{v}(\xi) \in \mathcal{O}\left(\left\{\xi ; \xi \in C^{1}-[0, c]\right\}\right)$.

(ii) $\hat{v}(\xi) \in \mathcal{O}\left(\left\{\xi \in \mathbb{C}^{1} ; 0<|\xi|<c\right\}\right)$ and

$$
\left\{\hat{v}(\xi)-\hat{v}\left(\xi e^{2 \pi i}\right)\right\} / 2 \pi i=g\left(\xi^{1 / \gamma}\right), \xi^{1 / \gamma}=|\xi|^{1 / \gamma} e^{i(\arg \xi) / \gamma} .
$$

Proof. From (10.8) we have (i). Since $g(z)$ is holomorphic in $\{|z|<R\}$, by deforming the integration path, we have the first assertion in (ii). Let $0<\xi<c$. Then, considering the holomorphic extension of $\hat{v}(\xi)$, we have

$$
\left\{\hat{v}(\xi)-\hat{v}\left(\xi e^{2 \pi i}\right)\right\} / 2 \pi i=\frac{1}{2 \pi i} \int_{z} \frac{e^{-a(z-\xi)} g\left(z^{1 / \gamma}\right)}{z-\xi} d z=g\left(\xi^{1 / \gamma}\right)
$$


where $Z=\{z(s) ; 0 \leqq s \leqq 1\}$ is a piecewise smooth contour such that $z(0)=z(1)=$ $0, z(1 / 2)=c, \operatorname{Im} z(s)<0$ for $0<s<1 / 2$ and $\operatorname{Im} z(s)>0$ for $1 / 2<s<1$ (see Fig. 10.1).

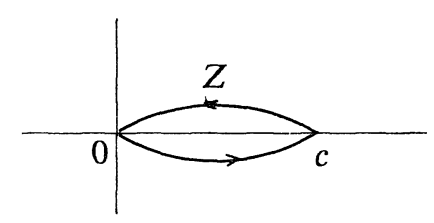

Fig. 10.1.

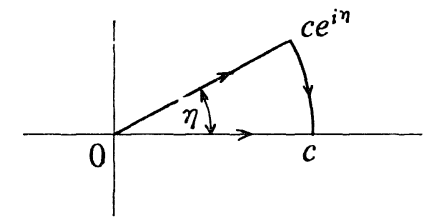

Fig. 10.2.

Remark 10.6. $\hat{v}(\xi)$ is represented for $\eta<\arg \xi<\eta+2 \pi$ in the form,

$$
\left\{\begin{array}{l}
\hat{v}(\xi)=\hat{v}_{\eta}(\xi)+\mathrm{a} \text { holomorphic function at } \xi=0, \\
\hat{v}_{\eta}(\xi)=\int_{0}^{c e^{i \eta}} \frac{e^{-a(z-\xi)} g\left(z^{1 / \eta}\right)}{z-\xi} d z
\end{array}\right.
$$

This follows from the deformation of the integration path. The holomorphic part of $\hat{v}(\xi)$ in $(10.10)$ corresponds to the integration from $c e^{i \eta}$ to $c$ in Fig. 10.2. Set

$$
v_{\eta}(t)=t^{\gamma} \int_{0}^{c e^{i \eta}} \exp \left(-t^{\gamma} z\right) g\left(z^{1 / \gamma}\right) d z, \quad 0<c^{1 / \gamma}<R .
$$

Then $v_{\eta}(t)$ has the $r$-asymptotic expansion

$$
v_{\eta}(t) \sim \sum_{k=0}^{+\infty} c_{k} t^{-k} \text { in } \tilde{S}(-(\pi / 2+\eta) / \gamma,(\pi / 2-\eta) / \gamma)
$$

and its $r$-Laplace transform is $\hat{v}_{\eta}(\xi)$,

$$
\hat{v}_{\eta}(\xi)=\int_{a}^{\infty e^{-i \eta}} \exp (\xi t) v_{\eta}\left(t^{1 / \eta}\right) d t
$$

Thus we obtain for $\hat{u}(\xi)=\hat{v}(\xi)+\hat{w}(\xi)$, the $r$-Laplace transform of $u(t)$ with the asymptotic expansion (10.1),

Proposition 10.7. (i) $\hat{u}(\xi)$ is holomorphic in $\{\xi ; \operatorname{Re} \xi<c, \xi \notin[0, c)\}$.

(ii) $\hat{u}(\xi)$ can be holomorphically extended into $\{\xi ; 0<|\xi|<c\}$ such that $\hat{u}(\xi) \in \mathcal{O}(\widetilde{\{\xi ; 0<|\xi|<c\}})$ and $\left\{\hat{u}(\xi)-\hat{u}\left(\xi e^{2 \pi i}\right)\right\} / 2 \pi i=g\left(\xi^{1 / \gamma}\right)$.

Next consider holomorphic functions on a sector. We have

Proposition 10.8. Let $u(t) \in \mathcal{O}\left(\tilde{S}\left(\theta_{0}\right)\right)$ and suppose that for any $\varepsilon>0$ there is $a C_{\mathrm{\varepsilon}}>0$ such that

$$
|u(t)| \leqq C_{\varepsilon} \exp \left(\varepsilon|t|^{\gamma}\right) \quad(\gamma>0)
$$


Then the followings hold:

(i) $\hat{u}(\xi) \in \mathcal{O}\left(\left\{\xi ;|\arg \xi-\pi|<r \theta_{0}+\pi / 2\right\}\right)$.

(ii) For any $\delta$ and $\varepsilon>0 \cdot \hat{u}(\xi) \mid \leqq M_{\varepsilon, \delta} \exp (a|\xi|)$ in $\left\{\xi ;|\arg \xi-\pi|<r \theta_{0}+\pi / 2-\delta,|\xi|>\varepsilon\right\}$.

(iii) If $u(t)$ is bounded on any closed subsector in $\tilde{S}\left(\theta_{0}\right)$, then $|\hat{u}(\xi)| \leqq C_{c, \delta}$ $|\log \xi|$ in $\left\{\xi ;|\arg \xi-\pi|<r \theta_{0}+\pi / 2-\delta, 0<|\xi|<c\right\}$.

Proof. By deforming the integration path in (1.12) in $\S 1$ we have (i) and the estimate in (ii). Let us show (iii). For $\xi \in\left\{\xi ;|\arg \xi-\pi|<\gamma \theta_{0}+\pi / 2-\delta\right\}$ we can choose $\omega$ such that $|\arg \xi+\omega-\pi|<\pi / 2-\delta / 2$ and $|\omega|<\gamma \theta_{0}-\delta / 2$. Putting $\arg t=\omega$, we have

$$
\begin{aligned}
& |\hat{u}(\xi)| \leqq M_{\delta}\left(\int_{a}^{a e^{i \omega}}+\int_{a e^{i \omega}}^{\infty e^{i \omega}}\right)\left|\exp (\xi t) t^{-1}\right||d t| \leqq \\
& \quad M_{\delta}\left\{\left(\exp (a|\xi|)+\int_{a e^{i \omega}}^{\infty e^{i \omega}} \exp \left(-c_{\delta}|\xi t|\right)|t|^{-1}|d t|\right) \leqq C_{c, \delta}|\log \xi| .\right.
\end{aligned}
$$

For $\hat{v}(\xi)$ defined by (10.8), the $r$-Laplace transform of $v(t)$, we have

Corollary 10.9. For any $\Theta>0$ there is a $M_{\Theta}$ such that

$$
|\hat{v}(\xi)| \leqq M_{\Theta}|\log \xi| \text { in }\{\xi ;|\arg \xi|<\Theta, 0<|\xi|<c / 2\} .
$$

Proof. $v_{\eta}(t)$ in Remark 10.6 has the $r$-asymptotic expansion in $\tilde{S}(-$ $(\pi / 2+\eta) / \gamma,(\pi / 2-\eta) / \gamma)$. The difference $\hat{v}(\xi)-\hat{v}_{\eta}(\xi)$ is holomorphic at $\xi=0$. So the assertion follows from Proposition 10.8-(iii).

Proof of Theorem 1.7. We can choose $c\left(0<c<R^{\gamma}\right)$ in Proposition 10.7 as close to $R^{\gamma}$ as possible (see (10.4)). So $\hat{u}(\xi)$ is holomorphic in $\left\{\xi ; \operatorname{Re} \xi<R^{\gamma}\right.$, $\left.\xi \notin\left[0, R^{\gamma}\right)\right\}, \hat{u}(\xi) \in \mathcal{O}\left(\left\{\overline{\left\{\xi 0<|\xi|<R^{\gamma}\right\}}\right)\right.$ and $\left\{\hat{u}(\xi)-\hat{u}\left(\xi e^{2 \pi i}\right)\right\} / 2 \pi i=g\left(\xi^{1 / \gamma}\right)$. From Corollary $10.9 \hat{u}(\xi)$ has the logarithmic growth at $\xi=0$.

Proof of Theorem 1.9. The only if part follows from Theorem 1.7. We show the conditions in Theorem 1.9 are sufficient. From the assumption, $\hat{u}(\xi)$ has at most the logarithmic growth at $\xi=0$. So by the deformation of the integration path (see Fig. 10.3) the inverse transform is given by

$$
\begin{aligned}
u(t) & =\frac{t^{\gamma}}{2 \pi i}\left(\int_{\infty}^{0} e^{i(\pi+\varphi)}+\int_{0}^{\infty} e^{i(\pi-\varphi)}\right) \exp \left(-\xi t^{\gamma}\right) \hat{u}(\xi) d \xi \\
= & \frac{t^{\gamma}}{2 \pi i}\left(\int_{c e^{2 \pi i}}^{0}+\int_{0}^{c}\right) \exp \left(-\xi t^{\gamma}\right) \hat{u}(\xi) d \xi+s_{\varphi}(t) \\
= & t^{\gamma} \int_{0}^{c} \exp \left(-\xi t^{\gamma}\right) F(\xi) d \xi+s_{\varphi}(t),
\end{aligned}
$$


where

$$
\left\{\begin{array}{l}
F(\xi)=\hat{u}(\xi)-\hat{u}\left(\xi e^{2 \pi i}\right), \\
s_{\varphi}(t)=\frac{t^{\gamma}}{2 \pi i}\left(\int_{\infty}^{c e^{i(\pi+\varphi)}}+\int_{c}^{\infty e^{i(\pi-\varphi)}}\right) \exp \left(-\xi t^{\gamma}\right) \hat{u}(\xi) d \xi
\end{array}\right.
$$

and $\varphi$ is a constant with $\pi / 2<\varphi<r \theta_{0}+\pi / 2$ and $\varphi \leqq \pi$

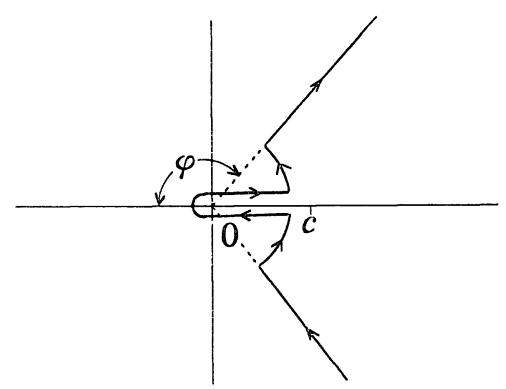

Fig. 10.3.

For $t \in \tilde{S}(\pi / 2 \gamma)$ we have the $r$-asymptotic expansion

$$
t^{\gamma} \int_{0}^{c} \exp \left(-\xi t^{\gamma}\right) F(\xi) d \xi \sim \sum_{k=0}^{+\infty} c_{k} t^{-k}
$$

and for any $\delta>0$ in $\left\{t ;|t| \geqq A^{\prime}, \pi / 2-\varphi+\delta<\gamma \arg t<-\pi / 2+\varphi-\delta\right\}$

$$
\left|s_{\varphi}(t)\right| \leqq A_{\varphi, \delta} \exp \left(-c_{\delta}|t|^{\gamma}\right) \quad\left(c_{\delta}>0\right) \text {. }
$$

Hence if $\theta_{0} \leqq \pi / 2 \gamma$, since we can choose $\varphi$ arbitrarily in $\left(\pi / 2, r \theta_{0}+\pi / 2\right), u(t)$ has the expansion (1.20). If $\theta_{0}>\pi / 2 \gamma$, we can put $\varphi=\pi$. We also have, choosing $\omega$ with $|\omega+r \arg t|<\pi / 2$ and $|\omega|<r \theta_{0}-\pi / 2$,

$$
u(t)=t^{\gamma} \int_{0}^{\infty} e^{i \omega} \exp \left(-\xi t^{\gamma}\right) F(\xi) d \xi
$$

Hence we get also the $r$-asymptotic expansion (1.20). Further assume $\theta_{0}>\pi / 2 \gamma+\pi$. Then this means $u\left(t e^{\pi i}\right)-u\left(t e^{-\pi i}\right)\left(|\arg t|<\theta_{0}-\pi\right)$ has the zero $\gamma$-asymptotic expansion at $t=\infty$ in $\tilde{S}\left(\theta_{0}-\pi\right)$, that is, for $z \in S\left(\theta_{1}\right)\left(0<\theta_{1}<\theta_{0}-\pi\right)$

$$
\left|u\left(t e^{\pi i}\right)-u\left(t e^{-\pi i}\right)\right| \leqq A B^{-N} \Gamma(N / \gamma+1)|t|^{-N} \quad \text { for any } N .
$$

This implies that there is a $C=C\left(\theta_{1}\right)>0$ such that for $t \in \tilde{S}\left(\theta_{1}\right)$

$$
\left|u\left(t e^{\pi i}\right)-u\left(t e^{-\pi i}\right)\right| \leqq A \exp \left(-C|t|^{\gamma}\right) .
$$

Since $\theta_{0}-\pi>\pi / 2 \gamma, u\left(t e^{\pi i}\right)-u\left(t e^{-\pi i}\right) \equiv 0$. Thus $u(t)$ is single valued in $\{t ;|t|>$ 
A\} and bounded at $t=\infty$. Hence $u(t)$ is holomorphic at $t=\infty$. This completes the proof of Theorem 1.9 .

Finally let us give

Proposition 10.10. Let $K(z, \lambda)$ be a continuous function defined on $\{(z, \lambda) \in$ $\left.\mathbb{C}^{n+1} \times \mathbb{C} ;|z| \leqq r, \lambda=\lambda e^{i \psi},|\lambda| \geqq \Lambda\right\}$, which is holomorphic in $z$ and fulfills $|K(z, \lambda)| \leqq A \exp \left(-c|\lambda|^{\alpha}\right)(c>0,0<\alpha<1)$. Then

$$
k(z)=\int_{\Lambda e^{i \psi}}^{\infty e^{i \psi}} \exp \left(\lambda z_{0}\right) K(z, \lambda) d \lambda
$$

has the asymptotic expansion with respect to $z_{0}$ in $U=\left\{|z| \leqq r ; \pi / 2<\arg z_{0}+\psi<\right.$ $3 \pi / 2\}$, that is,

$$
\left|k(z)-\sum_{n=0}^{N-1} k_{n}\left(z^{\prime}\right)\left(z_{0}\right)^{n} / n !\right| \leqq A B^{N} \Gamma(N / \gamma+1)\left|z_{0}\right|^{N},
$$

where $k_{n}\left(z^{\prime}\right)=\lim _{z_{0} \rightarrow 0} \in \in U\left(\partial / \partial z_{0}\right)^{n} k(z)$ and $\gamma=\alpha /(1-\alpha)$

Proof. We have

$$
\left(\partial / \partial z_{0}\right)^{n} k(z)=\int_{\Lambda e^{i \psi}}^{\infty e^{i \psi}} \sum_{k=0}^{n}\left(\begin{array}{c}
n \\
k
\end{array}\right) \lambda^{k} \exp \left(\lambda z_{0}\right)\left(\partial / \partial z_{0}\right)^{n-k} K(z, \lambda) d \lambda .
$$

Hence if $\pi / 2<\arg z_{0}+\psi<3 \pi / 2$, we have

$$
\begin{aligned}
& \left|\left(\partial / \partial z_{0}\right)^{n} k(z)\right| \leqq A \sum_{k=0}^{n} \int_{\Lambda e^{i \psi}}^{\infty}(n ! / k !) r^{k-n}\left|\lambda^{k}\right| \exp \left(-c|\lambda|^{\alpha}\right)|d \lambda| \\
& \quad \leqq A B^{n} \Gamma\left(\frac{n+1}{\alpha}\right) .
\end{aligned}
$$

By the Taylor's formula, we have

$$
\left|k(z)-\sum_{n=0}^{N-1} k_{n}\left(z^{\prime}\right)\left(z_{0}\right)^{n} / n !\right| \leqq A B^{N} \frac{\Gamma(N+1 / \alpha)}{N !}\left|z_{0}\right|^{N} \leqq A B^{N} \Gamma(N / \gamma+1)\left|z_{0}\right|^{N},
$$

where $k_{n}\left(z^{\prime}\right)=\lim _{z_{0} \rightarrow 0} \in U\left(\partial / \partial z_{0}\right)^{n} k(z)$ and $r=\alpha /(1-\alpha)$.

We can say for $k(z)$ defined by $(10.20)$ that $k\left(t^{-1}, z^{\prime}\right)$ has the $r$-asymptotic expansion with respect to $t$ in $\pi / 2<-\arg t+\psi<3 \pi / 2$.

\section{References}

[1] De Paris, J.C. Problème de Cauchy analytique à données singulières pour un opérateur différentiel bien decomposable, J. Math. Pures Appl., $5 \mathbb{1}$ (1972), 465-488.

[2] Hamada, Y., The singularities of solutions of Cauchy problem, Publ. RIMS, Kyoto Univ., 5 (1969), 21-40. 
[3] Hamada, Y., Leray, J. et C. Wagschal, Systèmes d'équations aux derivées partielles à caractéristiques multiples; problème de Cauchy ramifié; hyperbolicité partielle, $J$. Math. Pures Appl., 55 (1976), 297-352.

[4] Kashiwara, M. and Schapira, P., Problème de Cauchy pour les systèmes microdifférentiels dans le domain complexe, Inv. Math., 46 (1978), 17-38.

[5] Komatsu, H., Irregularity of characteristic elements and construction of null solutions, J. Fac. Sci. Univ. Tokyo Sec. IA Math., 23 (1976), 297-342.

[6] Ōuchi, S., Asymptotic behavior of singular solutions of linear partial differential equations in the complex domain, J. Fac. Sci. Univ. Tokyo Sec. IA Math., 27 (1980), $1-36$.

[7] —, An integral representation of singular solutions of linear partial differential equations in the complex domain, J. Fac. Sci. Univ. Tokyo Sec. IA Math., 27 (1980), $37-85$.

[8] — Characteristic Cauchy problems and solution of formal power series, Ann. Inst. Fourier, 33 (1983), 131-176.

[9] — Characteristic indices and subcharacteristic indices of surfaces for linear partial differential operators, Proc. Jap. Acad. Ser., 57A (1981), 481-484.

[10] — Index, localization and classification of characteristic surfaces for linear partial differential operators, Proc. Jap. Acad., 60A (1984), 189-192.

[11] - Existence of singular solutions and null solutions for linear partial differential operators, J. Fac. Sci. Univ. Tokyo Sec. IA Math., 32 (1985), 457-498.

[12] _ Solutions with singularities on a surface of linear partial differential equations, Proc. Hyperbolic equations and related topics, Taniguchi Symp. 1984, Kinokuniya Tokyo, 307-316.

[13] Persson, J., On the Cauchy problem in $\mathbb{C}^{n}$ with singular data, Mathematiche, 30 (1975), 339-362.

[14] - Singular holomorphic solutions of linear partial differential equations with holomorphic coefficients and nonanalytic solutions of equations with analytic coefficients, Astérisque 89-90, Soc. Math. France, (1981) 223-247.

[15] Ramis, J.P., Dévissage Gevrey, Astérisque 59-60, Soc. Math. France, (1978) 173-204.

[16] Wagschal, C., Problème de Cauchy analytique à données méromorphes, J. Math. Pures Appl., 51 (1972), 375-397. 
